\title{
Simulations of Transport in Hard Particle Systems
}

\author{
Pablo I. Hurtado ${ }^{1}$ (D) Pedro L. Garrido ${ }^{1}$
}

Received: 4 November 2019 / Accepted: 12 December 2019 / Published online: 2 January 2020

(c) Springer Science+Business Media, LLC, part of Springer Nature 2020

\begin{abstract}
Hard particle systems are among the most successful, inspiring and prolific models of physics. They contain the essential ingredients to understand a large class of complex phenomena, from phase transitions to glassy dynamics, jamming, or the physics of liquid crystals and granular materials, to mention just a few. As we discuss in this paper, their study also provides crucial insights on the problem of transport out of equilibrium. A main tool in this endeavour are computer simulations of hard particles. Here we review some of our work in this direction, focusing on the hard disks fluid as a model system. In this quest we will address, using extensive numerical simulations, some of the key open problems in the physics of transport, ranging from local equilibrium and Fourier's law to the transition to convective flow in the presence of gravity, the efficiency of boundary dissipation, or the universality of anomalous transport in low dimensions. In particular, we probe numerically the macroscopic local equilibrium hypothesis, which allows to measure the fluid's equation of state in nonequilibrium simulations, uncovering along the way subtle nonlocal corrections to local equilibrium and a remarkable bulk-boundary decoupling phenomenon in fluids out of equilibrium. We further show that the the hydrodynamic profiles that a system develops when driven out of equilibrium by an arbitrary temperature gradient obey universal scaling laws, a result that allows the determination of transport coefficients with unprecedented precission and proves that Fourier's law remains valid in highly nonlinear regimes. Switching on a gravity field against the temperature gradient, we investigate numerically the transition to convective flow. We uncover a surprising two-step transition scenario with two different critical thresholds for the hot bath temperature, a first one where convection kicks but gravity hinders heat transport, and a second critical temperature where a percolation transition of streamlines connecting the hot and cold baths triggers efficient convective heat transport. We also address numerically the efficiency of boundary heat baths to dissipate the energy provided by a bulk driving mechanism. As a bonus track, we depart from the hard disks model to study anomalous transport in a related hard-particle system, the $1 d$ diatomic hard-point gas. We show unambiguously that the universality conjectured for anomalous transport in $1 d$ breaks down for this model, calling into question recent theoretical predictions and offering a new perspective on anomalous transport in low dimensions. Our results show how carefully-crafted numerical simulations
\end{abstract}

Dedicated to Joel L. Lebowitz to celebrate his key role as leading editor of the Journal of Statistical Physics.

Communicated by Ivan Corwin.

Extended author information available on the last page of the article 
of simple hard particle systems can lead to unexpected discoveries in the physics of transport, paving the way to further advances in nonequilibrium physics.

Keywords Nonequilibrium statistical physics · Fourier's law $\cdot$ Local equilibrium · Convection $\cdot$ Anomalous transport $\cdot$ Molecular dynamics

\section{Introduction}

Understanding the physics of systems driven out of equilibrium remains as a main challenge of theoretical physics. In contrast with equilibrium statistical physics, there is yet no general bottom-up approach capable of predicting nonequilibrium macroscopic behavior in terms of microscopic physics [1-3], a direct result of the key role of dynamics out of equilibrium, even for nonequilibrium steady states [4,5]. This is a major drawback in our ability to control and engineer many natural and artificial systems which typically function out of equilibrium. A key feature of most nonequilibrium systems is transport, i.e. the appearance of nonzero currents of locally-conserved observables (as e.g. mass, momentum or energy) in response to external or internal driving mechanisms. Discerning how microscopic dynamics determines the long-time averages and fluctuations of these currents is naturally a central question in nonequilibrium statistical physics [3-5]. This problem has proven to be challenging, and up to now only few exactly solvable cases (mainly for simplified stochastic lattice models $[4,5])$ are fully understood. Nonequilibrium thermodynamics teaches us that, at least close to equilibrium, the steady-state currents typical of nonequilibrium transport are proportional to the thermodynamic forces of the problem, given in terms of the gradients of the different hydrodynamic fields [6]. This is the case e.g. of Fourier's law of heat conduction, which establishes the proportionality between the heat current and the local temperature gradient in a material, with the proportionality factor defining the heat conductivity $\kappa$, a key material property. Itâs going to be 200 years since Fourier stated this seminal law [7], but its rigorous microscopic understanding still poses a formidable problem [8].

Given the daunting difficulty of studying the dynamical (transport) properties of systems with many degrees of freedom, it seems mandatory to formulate simplified models of reality that, while capturing the fundamental ingredients of real systems, are more easily tractable. Universality arguments suggest that the emergent transport properties are not sensitive to microscopic details, but only to global features such as dimensionality, symmetries, conservation laws, range of forces, etc. One of the simplest ways to simulate microscopically a fluid consists in modelling its constituent particles as impenetrable bodies undergoing ballistic motion in between elastic collisions with neighboring particles. Such oversimplied description picks up however the essential ingredient underlying the physics of a large class of fluids, namely the strong, short-distance repulsion between neighboring molecules. This short-range repulsion dominates the local and global emerging structures in the fluid, as well as the nature of interparticle correlations $[9,10]$. In this way, hard-particle models and their relatives capture the physics of a large class of complex phenomena, ranging from phase transitions or heat flow to metastability, glassy dynamics, jamming, or the physics of liquid crystals and granular materials, to mention just a few [8-31], hence defining one of the most successful, inspiring and prolific models of physics.

At the theoretical level, hard-particle models offer also many advantages. For instance, the homogeneity of the interaction potential $[9,32,33]$ leads to a density-temperature separability in their thermodynamic and transport properties (e.g. equation of state, transport coefficients, 
etc.) which simplifies considerably their analysis. Moreover, the low- and moderate-density transport properties of hard-particle systems are directly comparable with predictions from kinetic theory (either Boltzmann-type equations for low-density or ring-kinetic theory for higher densities) [19,34]. Hard-particle models are also useful as reference systems for perturbative theories of interacting particle systems [9,35,36], an idea already contained in Van der Waals equation of state. However, the real power of hard particle models is the possibility of studying efficiently their large-scale emergent properties in detailed computer simulations. These numerical experiments allow us to obtain valuable information on problems which would be intractable in other way, providing at the same time crucial insights to understand the complex nonequilibrium behavior of many-body systems. There exist an extensive literature on efficient event-driven molecular dynamics algorithms for hard-particle models $[37,38]$, which open the door to massive, long-time simulations with large numbers of particles which are usually unfeasible for more realistic models. This last feature turns out to be crucial for the purposes of this paper, as the phenomenology that we will address typically requires excellent statistics to pick up weak but crucial signals in a sea of noisy fluctuations.

Despite the many recent advances in the understanding of nonequilibrium physics in general and transport phenomena in particular, there remain a large set of open, pressing problems that need to be addressed to further progress in these fields. These problems include, but are not restricted to, the following questions:

- Is macroscopic local equilibrium a robust property far from equilibrium? Can we use it to measure with precision equilibrium properties in nonequilibrium simulations?

- Can we measure corrections to local equilibrium in fluids at the fluctuating level? What is the nature of these corrections and what do they tell us about nonequilibrium behavior, in particular non-locality?

- Are there universal scaling features in the inhomogeneous structures and hydrodynamic profiles that a many-body system develops when driven out of equilibrium? If so, can we use these universal features to characterize transport in these systems?

- What is the nature of the Rayleigh-Bénard convection instability at the microscopic level? What type of order parameter captures best the convection transition?

- How is heat transport in a fluid affected by the transition to convective flow? Are there different heat conduction regimes?

- Can boundary heat baths dissipate effectively the energy input of a bulk driving mechanism? Can this competition lead to a well-defined nonequilibrium steady state?

- What is the nature and microscopic origin of the transport anomaly in low-dimensional materials? Can we use scaling methods to characterize this anomaly?

- Moreover, is the heat transport anomaly of $1 d$ momentum-conserving fluids and crystals universal? Can we determine with precission the anomaly exponent?

The purpose of this paper is to address these and related important questions in nonequilibrium physics using detailed numerical simulations of hard particle systems as a main tool, together with a recently introduced scaling approach to transport that offers unprecedented precission in the determination of key transport observables [33,39].

We structure this paper as follows. In Sect. 2 we introduce the hard disks model, paying special attention to some details of the simulations as e.g. the definition of stochastic thermal walls, range of parameters used, measurements of local and global observables, as well as error analysis. Section 3 summarizes a number of important yet basic known properties of hard disks, some of which will be useful in the course of our simulations and data analysis. These include equilibrium results in terms of the (unknown) exact equation of state, the effect of a gravitational field on the equilibrium physics of hard disks, as well as hard disks hydrody- 
namics (Navier-Stokes equations) and the associated transport coefficients. We then proceed in Sect. 4 to test our numerical simulations in a thermodynamic equilibrium situation. In particular we measure pressure and density profiles under varying graviational fields to test data against the hydrostatic formulas. In Sect. 5 we give our first step into the nonequilibrium physics of hard disks by driving the system with a boundary temperature gradient in the absence of gravity. Our goal in this section is to probe numerically the macroscopic local thermodynamic equilibrium (MLTE) hypothesis, which plays an important role in physics, being at the heart of many successful mesoscopic theories, from the classical hydrodynamics or nonequilibrium thermodynamics to recent macroscopic fluctuation theory. In particular, we show that MLTE is a property much stronger than previously anticipated, even under important finite-size effects. This allows us to measure the hard disks equation of state in nonequilibrium simulations with an excellent accuracy comparable to the best equilibrium simulations, uncovering along the way subtle nonlocal corrections to local equilibrium and a remarkable bulk-boundary decoupling phenomenon in fluids out of equilibrium. In Sect. 6 we further show that the the hydrodynamic profiles that hard disks fluid develops when driven out of equilibrium by an arbitrary temperature gradient obey universal scaling laws, a result that allows the determination of transport coefficients with unprecedented precission and proves that Fourier's law remains valid in strongly nonlinear transport regimes. In particular, this scaling method allows us to measure the marginal anomaly of the heat conductivity predicted for hard disks. In Sect. 7 we investigate numerically the transition to convective flow in hard disks by switching on a gravity field $g$ opposite to the temperature gradient direction. We uncover a surprising two-step convection transition scenario with two different critical temperatures. When the hot bath temperature reaches the first critical point, convection kicks in (as shown by a structured velocity fields) but gravity results in hindered heat transport as compared to the gravity-free case. It is at the second (higher) critical temperature that a percolation transition of streamlines connecting the hot and cold baths triggers efficient convective heat transport. We also show here numerically the existence of universal master curves for hydrodynamic fields in convective flow, at least for large values of the hot bath temperature. Section 8 is devoted to an important and pervasive question in nonequilibrium physics: can bulk forcing be balanced by a boundary dissipation mechanism? We answer affirmatively this question using simulations of hard disks with Gaussian (deterministic) boundary thermostats and a constant bulk driving field. In Sect. 9 we depart from the hard disks model to study anomalous transport in a related hard-particle system, the $1 d$ diatomic hard-point gas. In particular, we show that the universality conjectured for anomalous transport in $1 d$ breaks down for this model, calling into question recent renormalization-group and mode-coupling predictions and offering a new perspective on anomalous transport in low dimensions. Finally, a summary of the main results of this paper, including a discussion on their implications and possible avenues of future research, is provided is Sect. 10. The appendices contain technical details that, for the sake of clarity, we have omitted in the main text.

We note that the results presented in this review paper have been discussed in more detail in other works by these authors, see Refs. [32,33,39-41].

\section{The Hard Disks Model}

As already discussed, hard disks systems and their relatives capture the physics of a large class of complex phenomena [8-31], providing also key insights on the problem of heat conduction out of equilibrium, a main theme in this work. 
In particular, in this work we will be interested in a two-dimensional system of $N$ hard disks moving in a square box of unit side $L=1$, see Fig. 1, possibly under the action of a constant external field $\mathbf{g}=(0,-g)$ (which of course includes the gravity-free case for $g=0)$. Each disk has unit mass $(m=1)$ and a radius $\ell$ chosen so that the global areal density or packing fraction $\eta \equiv N \pi \ell^{2} / L^{2}$ is fixed, i.e. $\ell=\left[\eta L^{2} /(N \pi)\right]^{1 / 2}$. This will allow us to perform detailed finite-size analysis as $N \rightarrow \infty$ at constant $\eta$ and fixed temperature gradient. The disks dynamics consists in two distinct parts, namely free flight and collisions. The equations of motion during the free flight intervals in between collisions are simply given by Newton's second law, $d \mathbf{v}_{i} / d t=\mathbf{g}$, with trivial solution

$$
\mathbf{r}_{i}(t)=\mathbf{r}_{i}(0)+\mathbf{v}_{i}(0) t+\frac{1}{2} \mathbf{g} t^{2}, \quad \mathbf{v}_{i}(t)=\mathbf{v}_{i}(0)+\mathbf{g} t,
$$

where $\mathbf{r}_{i}(0)$ and $\mathbf{v}_{i}(0)$ are the values of the $i^{\text {th }}$-disk position and velocity, respectively, right after its last collision event. Disks collisions can be of three different types, namely (i) elastic collisions with another disk, (ii) elastic collisions with reflecting boundaries (if any), and (iii) collisions with stochastic walls simulating boundary thermal baths. In case (i) two disks collide when their relative distance equals $2 \ell$. Linear momentum and kinetic energy are conserved in the collision, and we assume that the velocity component perpendicular to the vector joining the disks' centers is unaltered during the collision. In this way we neglect any internal rotational degrees of freedom for the disks. In case (ii), when the disk collides elastically with a boundary wall, it gets perfectly reflected, e.g. for a wall along the $x$-direction the collision implies that $v_{x} \rightarrow-v_{x}$ and $v_{y} \rightarrow v_{y}$, being $\mathbf{v}=\left(v_{x}, v_{y}\right)$ the disk velocity vector. Finally, when a disk collides with a stochastic thermal wall in case (iii), its new velocity is randomly drawn from a Maxwellian distribution $P_{0, L}^{(x, y)}\left(v_{x, y}\right)$ defined by the wall temperature $T_{0, L}$. For a stochastic wall along e.g. the $y$-direction we have

$$
\begin{aligned}
& P_{0, L}^{(x)}\left(v_{x}\right)=\frac{1}{\sqrt{2 \pi k_{B} T_{0, L}}} \exp \left(-\frac{v_{x}^{2}}{2 k_{B} T_{0, L}}\right), \\
& P_{0, L}^{(y)}\left(v_{y}\right)=\frac{\left|v_{y}\right|}{\sqrt{2 \pi k_{B} T_{0, L}}} \exp \left(-\frac{v_{y}^{2}}{2 k_{B} T_{0, L}}\right),
\end{aligned}
$$

with the additional constraint that the $y$-component of the velocity changes $\operatorname{sign}\left(k_{B}\right.$ is Boltzmann's constant). The above random process simulates in a highly efficient manner the flow of energy in and out of the system from an infinite, equilibrium reservoir at the chosen temperature. Note also that for any non-zero temperature gradient $\Delta T \equiv\left|T_{L}-T_{0}\right| / L$ we expect a net heat current flowing from the hot reservoir to the cold one, as given by Fourier's law [8]. We note that, in addition to reflecting or thermal boundaries, we will consider when necessary periodic boundary conditions along any of the directions. Furthermore, when investigating the efficiency of boundary dissipation in Sect. 7, we will introduce a new type of boundary bath, the deterministic Gaussian bath [41], capable of thermalizing the system while maintaining the time-reversibility of the deterministic microscopic dynamics, see Fig. 1.

As for the parameters used in the simulations reported in this paper, we will consider systems with $N \in[957,8838]$ hard disks so as to analyze in detail finite-size effects and the thermodynamic $(N \rightarrow \infty)$ limit of the transport phenomena of interest. Global packing fractions in the broad range $\eta \in[0.05,0.725]$ will be investigated, comprising from dilute gases to dense solid-like behavior. Moreover, when studying the effect of temperature gradients, we will consider boundary temperatures $T_{0} \in[2,20]$ and $T_{L}=1$. We will also study the effect of gravity on transport by using external field values $g=5,10$ and 15 , together with the gravity-free case $g=0$. Computer simulations of hard disks systems have numerous 

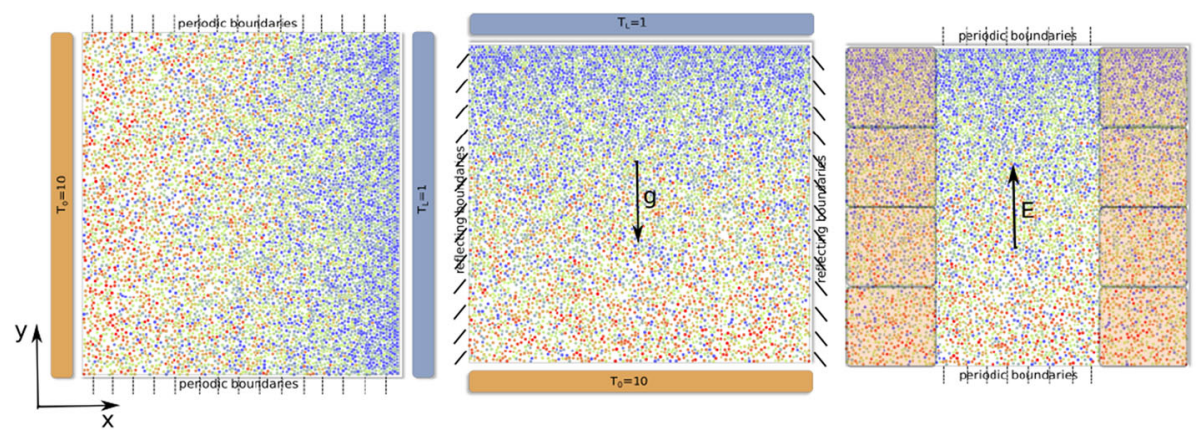

Fig. 1 Schematic view of the different hard disks systems simulated in this paper under varying driving protocols and boundary conditions. Left panel: hard disks fluid in contact with stochastic thermal walls at different temperatures $T_{0, L}$ along the $x$-direction and periodic boundary conditions along the $y$-direction in the absence of gravity. Central panel: hard disks under a gravity field of strength $g$ in the $y$-direction in contact with hot (cold) stochastic thermal wall at the bottom (top) of the simulation box, and subject to reflecting boundary conditions along the $x$-direction. Right panel: hard disks system in contact with Gaussian thermostats in the $x$-direction. The disks in the bulk are accelerated in the periodic $y$-direction by a driving field $E$, while disks at the shaded lateral boxes act as deterministic thermal baths, keeping constant their total kinetic energies for all times. Disks centers collide elastically with walls (represented as thick black lines)

advantages. The disks microscopic dynamics is simple enough and can be implemented algorithmically with high efficiency and with arbitrary precision (see for instance $[37,42,43]$ ). Moreover, its purely kinetic structure makes possible to fix one of the system external parameters $\left(T_{0}, T_{L}, g\right)$ by just applying a time rescaling without affecting the system dynamics. In other words, if we rescale time, $t=\alpha t^{\prime}$, disks velocities are rescaled in turn by $v=v^{\prime} / \alpha$ so, reparametrizing the temperature of the thermal baths as $T_{0, L}^{\prime}=\alpha^{2} T_{0, L}$ and the gravity field as $g^{\prime}=\alpha^{2} g$, one can prove that the dynamical evolution of a system of disks with parameters $\left(T_{0}, T_{L}, g\right)$ is indistinguishable from that of a system with parameters $\left(T_{0}^{\prime}, T_{L}^{\prime}, g^{\prime}\right)$. In this way one can arbitrarily choose $\alpha=1 / \sqrt{T_{1}}$ in order to fix to 1 the temperature of one of the thermal baths. This trick reduces the number of external control parameter to just $\left(T_{0}, g\right)$, together with the global packing fraction $\eta$. In order to obtain the behavior of any observable for arbitrary values of $T_{L}$ one just should apply the inverse time rescaling to the dynamical variables defining the observable of interest.

Next we discuss some details of the simulations. Initially the disks are placed regularly on the box with an initial velocity vector of random orientation and modulus $\sqrt{T_{0}+T_{L}}$. We then evolve the system during $10^{3}-5 \times 10^{4}$ collisions per particle (depending on the system size; note that our time unit was set to one collision per particle on average) to guarantee that the correct steady state has been reached. Only then we begin measurements of the different observables of interest every 100 collisions per particle, for a total time of $10^{7}$ collisions per particle, thus collecting a total of $M \sim 10^{5}$ data for averaging each observable. We use a $3 \sigma$-convention for the errorbars, so observables have typical errors of $3 \sigma / \sqrt{M} \simeq 0.01 \sigma$ that suffices to analyze in detail the emerging behavior. Small corrections $(\sim 0.1 \%)$ due to the spatial discretization of local measurements are explicitly taken into account and subtracted (see Appendix A in Ref. [32] for a brief description of this technical issue).

Before ending this section some words on measurements are mandatory. In order to characterize the spatial structures of the different nonequilibrium steady states we will encounter in the course of this work, we will perform local measurements of different observables of interest. For that, we will divide the unit box into either $n_{c} \times n_{c}$ (virtual) square cells of side 
$\Delta=1 / n_{c}$ for measurements of two-dimensional structure, or rather into $n_{c}$ rows of width $\Delta=1 / n_{c}$ along the gradient direction for measurements of one-dimensional profiles. In particular, we will use $n_{c}=30$ for $2 d$ maps, while $n_{c}=15$ or 30 will be used for $1 d$ profiles, see below. In what follows we discuss with some detail the $2 d$ case, the $1 d$ case being equivalent. A given cell is characterized by a pair of integer indexes $(n, l)$, with $n, l=1, \ldots, n_{c}$. We will be interested in hydrodynamic magnitudes at a given macroscopic point $(x, y)$, with $x, y \in[0, L=1]$, that correspond to averages of some microscopic observable at cell $(n, l)$, with $x=(n-1 / 2) / n_{c}$ and $y=(l-1 / 2) / n_{c}$. In this way we use the center of each cell as the macroscopic position of the hydrodynamic fields. Let $a(r, v)$ be a microscopic observable depending on a set of particle positions $r_{i}$ and velocities $v_{i}$. This observable can be for instance the kinetic energy of a particle, $a\left(\mathbf{r}_{i}, \mathbf{v}_{i}\right)=\mathbf{v}_{i}^{2} / 2$, or the local potential energy of a particle, $a\left(\mathbf{r}_{i}, \mathbf{v}_{i}\right)=g r_{i, y}$ where $\mathbf{r}_{i}=\left(r_{i, x}, r_{i, y}\right)$. The extensive value of $a(r, v)$ on cell $(n, l)$ at time $t$ is given by

$$
A(n, l ; t)=\sum_{i: \mathbf{r}_{i}(t) \in B(n, l)} a\left(\mathbf{r}_{i}(t), \mathbf{v}_{i}(t)\right)
$$

where $B(n, l)$ is the spatial domain associated to cell $(n, l)$. The previous sum involves a number $N(n, l ; t)=\sum_{i: r_{i}(t) \in B(n, l)} 1$ of particles at cell $(n, l)$ at time $t$. We perform $M$ measurements of $A\left(n, l ; t_{k}\right)$ at equispaced times $t_{k}, k \in[1, M]$, during the system evolution at the stationary state. The (intensive) average per particle is now given by

$$
\langle a(n, l)\rangle_{M}=\frac{\sum_{k=1}^{M} A\left(n, l ; t_{k}\right)}{\sum_{k=1}^{M} N\left(n, l ; t_{k}\right)}
$$

Note that, when compared with the average of $A(n, l ; t) / N(n, l ; t)$ over time, the previous averaging method exhibits a better convergence to the limiting $M \rightarrow \infty$ ensemble value, yielding also smaller fluctuations for the same $M$. For the error analysis, we assume that the set $\left\{A\left(t_{k}\right)\right\}_{k=1}^{M}$ of $M$ measurements of a given (local or global) observable is decorrelated in time so that the law of large numbers applies. In this case we expect for the average value of $A$ in the large- $M$ limit

$$
\langle A\rangle_{M} \simeq\langle A\rangle_{\infty}+\sigma(A ; M) \xi
$$

where $\xi$ is a Gaussian random variable with zero mean and unit variance, and

$$
\langle A\rangle_{M}=\frac{1}{M} \sum_{k=1}^{M} A\left(t_{k}\right), \quad \sigma(A ; M)=\frac{1}{M} \sqrt{\sum_{k=1}^{M}\left[A\left(t_{k}\right)-\langle A\rangle_{M}\right]^{2}}
$$

In this work our estimate for the asymptotic ensemble average will be $\langle A\rangle_{\infty}=\langle A\rangle_{M} \pm$ $3 \sigma(A ; M)$, so $99.7 \%$ of the data are within the errorbars shown in the analysis below.

\section{A Crash Course on Hard Disks}

As argued above, the hard disks system can be considered as a paradigm in many-body physics. Despite having extremely simple dynamics, essentially ballistic motion in between elastic collisions with neighboring disks, the hard disks fluid displays a landscape of highly nontrivial properties as a result of intricate collective effects among its individual constituents. For instance, hard disks exhibit an intriguing double phase transition [16] as a function of the packing fraction, a two-step melting transition which has been clarified only recently in extensive computer simulations [44], with a first-order transition from the liquid phase to a 
hexatic phase, followed by a continuos hexatic-solid transition. Moreover, hard disks also exhibit the important and universal long-time tails in correlation functions $[38,45]$ which were first discovered by Alder and Wainwright for hard spheres [18] and characterize relaxation in most fluids. However it is worth noting that, despite the interest of the hard disks model, relevant equilibrium information as e.g. its exact equation of state $(\mathrm{EoS})$ has not yet been derived analytically in the context of the equilibrium statistical physics [9].

In this section we briefly review a few known basic properties of hard disks, some of which will be useful in the course of our simulations and data analysis below. We start with some equilibrium properties. The canonical partition function for a two-dimensional system of $N$ disks with mass $m$ and radius $\ell$ enclosed in a square box of side $L$ at a temperature $T$ is given by

$$
Z(N, S, T)=\frac{1}{h^{2 N} N !}\left(2 \pi m k_{B} T\right)^{N} Z_{c}(N, S),
$$

where $h$ and $k_{B}$ are Planck's and Boltzmann's constants, respectively (that will be set to 1 below by an appropriate choice of units), $S=L^{2}$ is the box surface and $Z_{c}$ is the configurational partition function that, for hard disks, doesn't depend on temperature. $Z_{c}(N, S)$ is not known in general but can be connected with the equation of state. Indeed, the hard disks EoS has been extensively studied numerically, and excellent analytical approximations have been obtained from these extensive computer simulations $[9,46]$. In particular, the hard disks EoS takes the general form

$$
P S=N k_{B} T H(\rho),
$$

with $P$ the local pressure and $\rho$ the local packing fraction or areal density. ${ }^{1}$ In this way all the complex structure of the EoS (which includes the aforementioned double phase transition) is mapped onto the unknown function $H(\rho)$. A simple and well-known phenomenological form for $H(\rho)$ was proposed by Henderson [47],

$$
H(\rho)=\frac{1+\rho^{2} / 8}{(1-\rho)^{2}},
$$

that fits well simulation data for a broad regime of areal densities in the interval $\rho \in[0,0.5]$, see also [46]. To connect the $\operatorname{EoS}$ with $Z_{c}$, we just note that

$$
P=k_{B} T \frac{\partial \ln Z_{c}}{\partial S} \Rightarrow N H(\rho)=-\rho \frac{\partial \ln Z_{c}}{\partial \rho}
$$

If we assume a scaling (large deviation) form $Z_{c} \simeq \exp \left[N f_{c}(\rho)\right]$ for large enough $N$, as expected from extensivity, with $f_{c}(\rho)$ some function of the packing fraction, we obtain

$$
f_{c}(\rho)=f_{c}\left(\rho^{*}\right)-\int_{\rho^{*}}^{\rho} d \phi \frac{H(\phi)}{\phi},
$$

where $\rho^{*}$ is some arbitrary packing fraction. We further expect that in the low density, $\rho \rightarrow 0$ limit the hard disk system will behave as an ideal gas. In such limit $P S=N k_{B} T$ and hence $f_{c}(0)=\ln S-\ln \rho=\ln \left(\pi \ell^{2} N / \rho\right)-\ln \rho$, so substituting $\rho^{*}=0$ above we get

$$
f_{c}(\rho)=\frac{1}{N} \ln Z_{c}=\ln \left(\frac{\pi \ell^{2} N}{\rho}\right)-\int_{0}^{\rho} d \phi \frac{H(\phi)-1}{\phi} .
$$

${ }^{1}$ For an homogeneous, equilibrium system both $P$ and $\rho$ are constant, with $\rho=\eta \equiv N \pi \ell^{2} / S$ the global packing fraction. 
Therefore the hard disk partition function can be written for $N$ large as $Z \simeq \exp [N f(\rho)]$, with

$$
f(\rho)=\ln \frac{T}{\rho}-\int_{0}^{\rho} d \phi \frac{H(\phi)-1}{\phi}+C,
$$

where $C$ is the constant

$$
C=\ln \left(\frac{2 m k_{B} e \pi^{2} \ell^{2}}{h^{2}}\right) .
$$

We can now compute all thermodynamic magnitudes of interest from Eq. (13), e.g. the entropy is given by

$$
\frac{s}{k_{B}}=f(\rho)+1
$$

while the specific heats at constant pressure and volume are, respectively,

$$
\frac{c_{P}}{k_{B}}=1+\frac{H(\rho)^{2}}{H(\rho)+\rho H^{\prime}(\rho)}, \quad c_{V}=k_{B},
$$

with $H^{\prime}(\rho)=d H(\rho) / d \rho$. The coefficients of thermal expansion at constant pressure $\left(\alpha_{P}\right)$ and isothermal compressibility $\left(\kappa_{T}\right)$ are in turn

$$
\alpha_{P}=\frac{1}{T} \frac{H(\rho)}{H(\rho)+\rho H^{\prime}(\rho)}, \quad \kappa_{T}=\frac{\pi \ell^{2}}{k_{B} T \rho} \frac{1}{H(\rho)+\rho H^{\prime}(\rho)},
$$

In the course of this paper we will measure the function $H(\rho)$ in extensive nonequilibrium molecular dynamics simulations, see below.

We will be also interested below in the physics of a hard disks fluid under the action of a gravitational field, e.g. in order to study the transition to convective flow and the Bénard problem. It is therefore useful to review the equilibrium properties of hard disks in the presence of an external potential, to e.g. derive the hydrostatic formula. With this idea in mind we now assume the presence of an external, position-dependent field $U(\mathbf{r})$ acting on the particles in $2 d$. This field thus modifies the Hamiltonian of the hard disks model with an additional term

$$
H_{\text {ext }}=\sum_{i=1}^{N} U\left(\mathbf{r}_{i}\right),
$$

where $\mathbf{r}_{i}$ is the position of the ith-disk. It is now convenient to work within the grand-canonical ensemble in order to obtain the equilibrium properties of the fluid under this external field. Moreover, the calculation is simplified if we consider a slowly-varying external field [48], i.e. a field $U(\mathbf{r})=\tilde{U}(\mathbf{r} / L)$ with $L$ the macroscopic linear size of the box and $\tilde{U}$ a smooth function in $\mathbb{R}^{2}$. We can define now a local cell with a volume that grows with $L^{2}$ around a given position $\mathbf{x}$. When $L \rightarrow \infty$ the particles of the bulk of the box only see an external constant field of value $\tilde{U}(\mathbf{x})$ and then $H_{\text {ext }}=N \tilde{U}(\mathbf{x})$. In this way the local thermodynamic properties of the system can be computed using the grand-canonical ensemble just substituting the chemical potential $\mu \rightarrow \mu-\tilde{U}(\mathbf{x})$ [48]. For instance, the EoS can be written in a parametric form

$$
\begin{aligned}
& P=k_{B} T a[T, \mu-\tilde{U}(\mathbf{x})], \\
& \tilde{\rho}=k_{B} T \frac{d}{d \mu} a[T, \mu-\tilde{U}(\mathbf{x})],
\end{aligned}
$$

where $\tilde{\rho}$ is the local particle density, $a=a(T, \mu)=\lim _{S \rightarrow \infty} S^{-1} \log \mathcal{Z}$, and $\mathcal{Z}$ is the grandcanonical partition function. Parameter $\mu$ can be now eliminated by applying the gradient 
to the pressure and using the definition of $\tilde{\rho}$, leading to the well-known hydrostatic formula [48]

$$
\nabla P=-\tilde{\rho}(\mathbf{x}) \nabla \tilde{U}
$$

which relates the pressure gradient in the presence of an external field with the associated particle density profile. It is interesting to note that the argument developed here is rather general and not restricted to a hard disks system.

Next we focus on the hard disks fluid hydrodynamics as described by the Navier-Stokes equations. This set of equations describes the local spatiotemporal dynamics of a macroscopic fluid [49] starting from the following assumptions: (1) local conservation of particle density, linear momentum and energy, (2) macroscopic local equilibrium, and (3) some local constitutive relations (Fourier's law, Newton relation) linking the currents of the different conserved quantities with the gradients of the hydrodynamic fields. While the first point holds true for any conservative fluid, the other two hypotheses, though reasonable, are strong statements on the nonequilibrium physics of the hard disks fluid that need further support. We will devote part of this paper to describe strong numerical evidences supporting these two hypotheses for hard disks and other hard-particle systems. The macroscopic hydrodynamic fields are then the local mass density, $\tilde{\rho}(x, t)$, the local velocity components, $u_{i}(x, t), i=1, \ldots, d$, and the local temperature, $T(x, t)$, and their time evolution obeys

$$
\begin{aligned}
\partial_{t} \tilde{\rho}+\sum_{i} \partial_{i}\left(\tilde{\rho} u_{i}\right) & =0 \\
\partial_{t} u_{i}+\sum_{j} u_{j} \partial_{j} u_{i} & =\frac{1}{\tilde{\rho}} \sum_{j} \partial_{j} \tau_{i j}+g_{i}, \\
\tilde{\rho} \tilde{c}_{v}\left(\partial_{t} T+\sum_{i} u_{i} \partial_{i} T\right) & =-\left.T \frac{\partial P}{\partial T}\right|_{\tilde{\rho}} \sum_{i} \partial_{i} u_{i}+\sum_{i j} \tau_{i j}^{\prime} \partial_{i} u_{j}+\sum_{i j} \partial_{i}\left(\kappa_{i j} \partial_{j} T\right),
\end{aligned}
$$

where $\tau_{i j}$ is the stress tensor, $g_{i}$ is the component of the external field acting along direction $i \in$ $[1, d], P$ is the pressure and $\kappa_{i j}$ is the thermal conductivity matrix. For so-called Newtonian fluids we can further write

$$
\begin{aligned}
\tau_{i j} & =-P \delta_{i j}+\tau_{i j}^{\prime}, \\
\tau_{i j}^{\prime} & =v\left(\partial_{i} u_{j}+\partial_{j} u_{i}\right)+v^{\prime} \delta_{i j} \sum_{k} \partial_{k} u_{k} .
\end{aligned}
$$

Moreover, the local equilibrium property implies that the equilibrium EoS can be used to relate locally the hydrodynamic fields, i.e. $P=P(\tilde{\rho}, T)$. In addition, different thermodynamic and transport coefficients need to be known, i.e. the equilibrium mass specific heat at constant volume $\tilde{c}_{v}=\tilde{c}_{v}(\tilde{\rho}, T)$, the thermal conductivity $\kappa=\kappa(\tilde{\rho}, T)$, the shear viscosity $v=v(\tilde{\rho}, T)$ and the second viscosity $v^{\prime}=v^{\prime}(\tilde{\rho}, T)$. The above equations, together with appropriate boundary conditions for the hydrodynamic fields, give a complete description of the time evolution of a fluid at the macroscopic level.

For hard disks $c_{v}=k_{B}$ and the EoS has a well-defined temperature dependence, see Eq. (8), so we can write

$$
\left.\frac{\partial P}{\partial T}\right|_{\tilde{\rho}}=\frac{P}{T} .
$$

Moreover, we can simply relate the areal and mass densities noting that $\rho=N \pi \ell^{2} / L^{2}$ while $\tilde{\rho}=N m / L^{2}$, so $\tilde{\rho}=m \rho / \pi \ell^{2}$ where $m$ is the mass of one disk and $\ell$ its radius. The 
density-temperature separability of the hard disks EoS, a scaling property stemming from the homogeneity of the hard disks interparticle potential (see Ref. [32]), is also inherited by the different transport coefficients. This leads to a simple $\sqrt{T}$ temperature dependence for these transport coefficients, namely

$$
\begin{aligned}
v & =\frac{\sqrt{k_{B} m}}{\ell} \sqrt{T} E(\rho), \\
v^{\prime} & =\frac{\sqrt{k_{B} m}}{\ell} \sqrt{T} E^{\prime}(\rho), \\
\kappa & =\frac{\sqrt{k_{B}^{3}}}{\ell \sqrt{m}} \sqrt{T} k(\rho) .
\end{aligned}
$$

We will be interested below in stationary, time-independent solutions to the Navier-Stokes equations for two-dimensional hard disks both in the absence of external fields, $g_{i}=0 \forall i$, or in the presence of gravity, $g_{i}=-g \delta_{i, 2}$. The resulting equations in this case read

$$
\begin{aligned}
\partial_{1}\left(\rho u_{1}\right)+\partial_{2}\left(\rho u_{2}\right)= & 0 \\
\rho\left(u_{1} \partial_{1} u_{1}+u_{2} \partial_{2} u_{1}\right)= & -\partial_{1} Q+\pi \ell \partial_{1}\left[\sqrt{T}\left(2 E \partial_{1} u_{1}+E^{\prime}\left(\partial_{1} u_{1}+\partial_{2} u_{2}\right)\right)\right] \\
& +\pi \ell \partial_{2}\left[\sqrt{T} E\left(\partial_{1} u_{2}+\partial_{2} u_{1}\right)\right] \\
\rho\left(u_{1} \partial_{1} u_{2}+u_{2} \partial_{2} u_{2}\right)= & -\partial_{2} Q+\pi \ell \partial_{2}\left[\sqrt{T}\left(2 E \partial_{2} u_{2}+E^{\prime}\left(\partial_{1} u_{1}+\partial_{2} u_{2}\right)\right)\right] \\
& +\pi \ell \partial_{1}\left[\sqrt{T} E\left(\partial_{1} u_{2}+\partial_{2} u_{1}\right)\right]-\rho g \\
\rho\left(u_{1} \partial_{1} T+u_{2} \partial_{2} T\right)= & -Q\left(\partial_{1} u_{1}+\partial_{2} u_{2}\right)+2 \pi \ell \sqrt{T} E\left(\left(\partial_{1} u_{1}\right)^{2}+\left(\partial_{2} u_{2}\right)^{2}\right) \\
& +\pi \ell \sqrt{T} E^{\prime}\left(\partial_{1} u_{1}+\partial_{2} u_{2}\right)^{2} \\
& +\pi \ell \sqrt{T} E\left(\partial_{1} u_{2}+\partial_{2} u_{1}\right)^{2} \\
& +\pi \ell\left[\partial_{1}\left(\sqrt{T} k \partial_{1} T\right)+\partial_{2}\left(\sqrt{T} k \partial_{2} T\right)\right]
\end{aligned}
$$

where we have defined the reduced pressure, $Q \equiv \pi \ell^{2} P$, and we note that $E, E^{\prime}$ and $k$ are functions of $\rho$. Note also that the following mapping has been used to simplify the previos equations

$$
k_{B} T \rightarrow T, \quad m g \rightarrow g, \quad \sqrt{m} u_{1,2} \rightarrow u_{1,2} .
$$

With this mapping the EoS can be simply written as

$$
Q=T q(\rho)=T \rho H(\rho)
$$

Observe that we use in all equations above the areal density $\rho$ instead of the mass density $\tilde{\rho}$, though both choices are equally valid. Interestingly, the Navier-Stokes equations for hard disks simplify substantially when considering non-convective stationary solutions. In this case the hydrodynamic fields can be written as

$$
\rho=\rho(y), \quad u_{1}=u_{2}=0, \quad T=T(y),
$$

and are the solutions of the following set of simplified equations

$$
\frac{d Q}{d y}=-g \rho,
$$




$$
\begin{aligned}
\sqrt{T} k(\rho) \frac{d T}{d y} & =-J, \\
Q & =T q(\rho),
\end{aligned}
$$

together with the constraints imposed by boundary conditions and the conservation of the total mass,

$$
T(0)=T_{0}, \quad T(1)=T_{L}, \quad \eta=\int_{0}^{1} \rho(y) d y
$$

where $T_{0, L}, g$ and $\eta$ (the global packing fraction) are control parameters for the problem at hand. Note that the heat current $J$ in Eq. (32) is a constant determined as a function of the external parameters.

To end this section, we note that exact expressions for the hard disks transport coefficients have not been derived so far. However, approximate expressions can be derived within the Enskog approximation of kinetic theory. In particular, Gass obtained the following expressions for the shear viscosity $v$, the bulk viscosity $\zeta$ and the thermal conductivity $\kappa$ using Enskog theory [34]

$$
\begin{aligned}
& v(\rho, T)=\frac{\sqrt{k_{B} m}}{\ell} \sqrt{T} E(\rho), \quad \text { with } E(\rho)=\frac{0.255}{\sqrt{\pi} \chi(\rho)}\left[1+2 \rho \chi(\rho)+3.4197 \rho^{2} \chi(\rho)^{2}\right] \\
& \zeta(\rho, T)=\frac{\sqrt{k_{B} m}}{\ell} \sqrt{T} E_{B}(\rho), \quad \text { with } E_{B}(\rho)=1.2734 \rho^{2} \chi(\rho) \\
& \kappa(\rho, T)=\frac{\sqrt{k_{B}^{3}}}{\ell \sqrt{m}} \sqrt{T} k(\rho), \quad \text { with } k(\rho)=\frac{1.029}{\chi(\rho)}\left[1+3 \rho \chi(\rho)+3.4874 \rho^{2} \chi(\rho)^{2}\right]
\end{aligned}
$$

where the function $\chi(\rho)$ is defined as follows

$$
\chi(\rho) \equiv \frac{H(\rho)-1}{2 \rho}
$$

and the second viscosity is $v^{\prime}=\zeta-v$.

\section{Equilibrium First: Some Simulation Results}

Before addressing the numerical characterization of transport in hard disks and the associated nonequilibrium steady states, we want to test our numerical simulations in a thermodynamic equilibrium situation. As described in Sect. 3, for a hard disks system in equilibrium at temperature $T$ and possibly under a gravitational field $g$, the (local) pressure and density are related by the equation of state and the hydrostatic formula, i.e.

$$
\begin{aligned}
Q(y) & =T \rho(y) H(\rho(y)) \\
\frac{d Q(y)}{d y} & =-g \rho(y)
\end{aligned}
$$

where $Q(y)=P(y) \pi \ell^{2}$ is the reduced pressure at height $y$, already introduced in Sect. 3 , and we recall that the disks mass is taken as $m=1$. These equations, together with the appropriate boundary conditions, determine the behavior of $Q$ and $\rho$ as a function of $y$. Of course, the exact expression for $H(\rho)$ in hard disks is unknown, although Henderson's formula $H(\rho)=\left(1+\rho^{2} / 8\right) /(1-\rho)^{2}$ is known to be an excellent approximation in the 
range $\rho \in[0,0.5]$, with a relative error below $1 \%$ in this regime $[32,47]$. Using Henderson's EoS to solve the above equations we obtain

$$
\begin{aligned}
Q(y) & =T \rho \frac{1+\rho(y)^{2} / 8}{[1-\rho(y)]^{2}} \\
y & =-\frac{1}{g}\left[\log \rho(y)-\frac{7}{8} \log (1-\rho(y))+\frac{7}{8(1-\rho(y))}+\frac{9}{8(1-\rho(y))^{2}}+C\right]
\end{aligned}
$$

where the constant $C$ is fixed by giving the density at a particular height, $\left(y_{0}, \rho_{0}\right)$. The purpose of this brief section is to test numerical results in equilibrium for varying external fields $g$ against these predictions.

For that we measured the stationary density and pressure profiles along the $y$-direction for $N=957$ hard disks at a global packing fraction $\eta=N \pi \ell^{2} / L^{2}=0.2$ in equilibrium with temperature $T_{0}=T_{L}=1$ at the top and bottom stochastic thermal walls, and with reflecting walls along the $x$-direction, see central panel in Fig. 1 . In the simulations, the local areal density $\rho(n, l)$ at cel $(n, l)$ is computed as follows, see Sect. 2,

$$
\rho(n, l)=\frac{N(n, l) \pi \ell^{2}}{\Delta^{2}}
$$

where $N(n, l)$ is the average number of disks with its center within cell $(n, l)$ at the stationary state, $\ell$ is the disk radius, and $\Delta=1 / n_{c}$ is the linear size of the cell (in this case we take $n_{c}=30$ virtual rows so $\left.\Delta=1 / 30\right)$. This definition for $\rho(n, l)$, though very efficient from a computational point of view, leads to systematic deviations of the areal density near the system boundaries due to surface exclusion around the walls. Therefore, in order to minimize these spurious effects, we exclude from our analysis data from both boundary cells and their neighboring cells, i.e. two cell layers besides the boundaries. Moreover, to obtain density and pressure profiles from the two-dimensional data grid, we average the results measured for all cells at each constant height (i.e. at constant cell index $l$ ).

In order to measure the reduced pressure field $Q(x, y)=\pi \ell^{2} P(x, y)$, we use the virial theorem [1]. Appendix A discusses the derivation of the virial pressure in the case of hard disks. For any non-zero gravitational field, the equilibrium fluid will develop a non-trivial spatial structure for both density and pressure, see Eqs. (39)-(40). Hence, assuming that the conditions to derive the hydrostatic formula hold, see Sect. 3, we can use the hard-disks virial formula locally for the pressure, see Appendix A. In this case

$$
Q(x, y)=T(x, y) \rho(x, y)+\frac{\pi \ell^{2}}{2 \Delta^{2} \tau_{\mathrm{col}}}\left\langle\sum_{\operatorname{col}(\mathrm{x}, \mathrm{y})} \mathbf{v}_{i j} \cdot \mathbf{r}_{i j}\right\rangle,
$$

where $\rho(x, y)$ is the local packing fraction, $T(x, y)$ is the temperature field which in this equilibrium case is just uniform, $T(x, y)=T_{0}=T_{L}=1$, and the sum is taken over all collisions occurring during a time interval $\tau_{\text {col }}$ in a cell centered at a macroscopic position $(x, y)$. Moreover, $\mathbf{v}_{i j}=\mathbf{v}_{i}-\mathbf{v}_{j}$ is the relative velocity of the colliding pair $(i, j), \mathbf{r}_{i j}$ is the vector connecting the particles centers at collision, with $r_{i j}=2 \ell$, and angular brackets represent an ensemble average. We will show below that, when driven out of equilibrium by a boundary temperature gradient, the resulting nonequilibrium steady state obeys with high accuracy the macroscopic local equilibrium property described in Sect. 3. This implies in particular that the local virial expression for the pressure, Eq. (42), yields correct values even far from equilibrium, consistent with other measurements as e.g. the pressure exerted by the fluid on the walls. More on this issue in Sect. 5 below. 


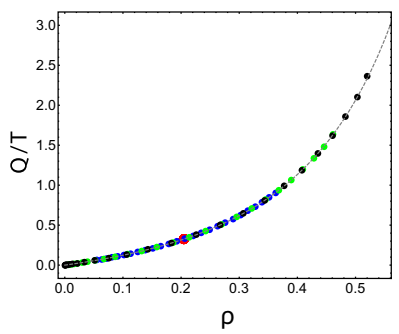

(a)

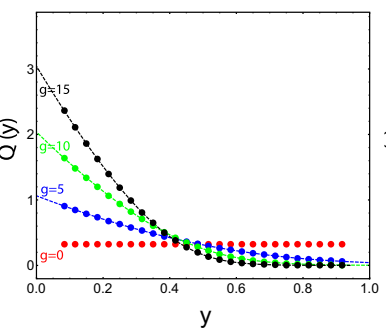

(b)

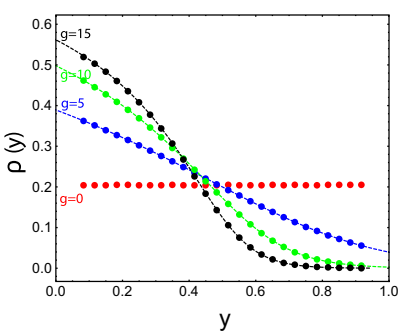

(c)

Fig. 2 Equilibrium behavior of hard disks for $T_{0}=T_{L}=1$, global packing fraction $\eta=0.2$, and varying gravitational fields $g$. a Equation of State (EoS): The measured reduced pressure $Q(y) / T$ versus $\rho(y)$ for $g=0$ (red big dot), $g=5$ (blue dots), $g=10$ (green dots) and $g=15$ (black dots). b Density profile along the $y$-direction for different values of $g$. c Virial pressure profile for varying $g$. Dashed lines are the predictions using the hydrostatic formula and the Henderson's EoS for $H(\rho)$, Eqs. (39)-(40) (Color figure online)

Figure 2 shows the results obtained for $\eta=0.2, T_{0}=T_{L}=1$ and varying values for $g$, as well as a comparison with predictions based on Henderson's formula. In particular, Fig. 2a shows the measured values of $Q(y) / T$ as a function of the local average density $\rho(y)$ for all different $g$ 's. All data collapse onto a universal curve $\rho H(\rho)$ defined by the hard disks EoS, see Eq. (8), and this scaling curve agrees with high accuracy with Henderson's prediction in the range of local packing fractions explored, see dashed line in Fig. 2a. This agreement confirms the validity of the hypothesis behind the hydrostatic formula, even for the small cell size we use for local measurements in these simulations. This is probably due to the strong chaoticity of disks dynamics, which makes spatial and temporal correlations to decay fast enough. On the other hand, Fig. 2b, c show the measured reduced pressure and density profiles, respectively, for different values of the external field $g$. In all cases the measured profiles follow closely the hydrostatic formulas obtained with the Henderson EoS, confirming again the empirical validity of this approximation in the density range explored. Overall, these equilibrium results and their successful comparison with theoretical predictions validate our numerical simulations.

The next natural step is hence to investigate numerically the nonequilibrium steady states that the hard disks fluid displays when driven by a temperature gradient, both with or without gravity. This is the purpose of next sections. We start by considering a driven hard disks fluid in the absence of gravity, where the typical nonequilibrium steady state is described by the paradigmatic Fourier's law of heat transport. We leave the effect of gravity on nonequilibrium transport for later sections, where we will characterize numerically the transition to convective flow and the emergence of spatiotemporal roll structures.

\section{Probing Macroscopic Local Equilibrium}

Our first step into the nonequilibrium physics of hard disks will consists in driving the system with a boundary temperature gradient in the absence of gravity, so $g=0$ in this and next section (later on in Sect. 7 we will switch on gravity to study convective flow). To stress the lack of gravity effects in this section, we will consider a hard disks system subject to a temperature gradient along the $x$-direction imposed by stochastic thermal walls at different temperatures $T_{0} \neq T_{L}=1$ located at $x=0$ and $x=L$, respectively, together with periodic boundary conditions along the $y$-direction to minimize (unavoidable) boundary effects, see Fig. 3 . 


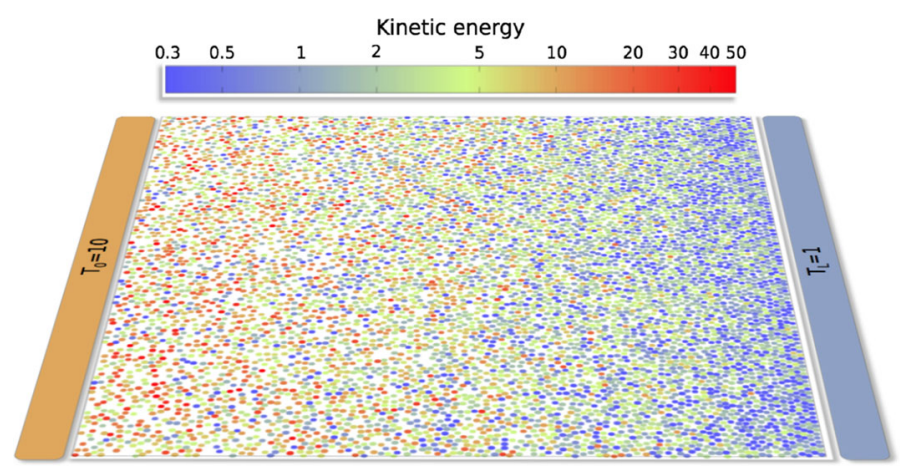

Fig. 3 Snapshot of a typical configuration with $N=7838$ hard disks at global packing fraction $\eta=0.5$, subject to a strong temperature gradient $\left(T_{0}=10, T_{L}=1\right)$ along the $x$-direction, and with periodic boundary conditions along the $y$-direction. In these simulations there is no gravity, so $g=0$, see below. Colors represent kinetic energy as described in the bar legend (Color figure online)

In this situation, given the initial state and after a safe relaxation time, the hard disks system will reach a nonequilibrium steady state (NESS) characterized by a net heat current flowing from the hot to the cold reservoir, and an inhomogeneous spatial structure for the temperature and density hydrodynamic fields. This structure can be obtained by solving the (simplified) Navier-Stokes equations for this problem, see Sect. 3, if exact (or approximate) expressions for the transport coefficients in terms of the hydrodynamic fields are known. In contrast with equilibrium, the microscopic probability measure associated to such a NESS (or mNESS hereafter) will be typically a complex object, often defined on a fractal support dubbed strange attractor in literature. Moreover, as oppossed to equilibrium, this microscopic probability measure is usually very sensitive to microscopic details such as the modeling of boundary reservoirs (e.g. stochastic vs deterministic) [50-58], and the connection between the mNESS and a nonequilibrium analog of thermodynamic potentials is far from clear nowadays.

On the other hand, it is well-known that essentially different mNESSs (resulting from e.g. different modelings of boundary baths) describe equally well what seems to be the same macroscopic NESS (or MNESS in short) [28,59]. This macroscopic NESS is defined in terms of a few macroscopically smooth hydrodynamic fields, solution of the aforementioned Navier-Stokes equations for this problem, see Eqs. (24)-(27) for the particular case of hard disks. A cornerstone for this sort of nonequilibrium ensemble equivalence, which can be formally stated via the chaotic hypothesis of Gallavotti and Cohen [55-58], is the notion of local thermodynamic equilibrium (LTE) [50,60]. This refers to the fact that an interacting nonequilibrium system reaches locally an equilibrium-like state defined by e.g. a local temperature, density and velocity (the first two related locally via standard thermodynamic relations), which are roughly constant across molecular scales but change smoothly at much larger macroscopic scales, where their evolution is governed by hydrodynamic equations. LTE plays an important role in physics, being at the heart of many successful theories, from the classical hydrodynamics $[61,62]$ or nonequilibrium thermodynamics [6] to recent macroscopic fluctuation theory (MFT) [4,5,27,63-66]. Despite this success, LTE is not capable of explaining some intriguing nonequilibrium phenomenology. In particular, this is the case for one of the main hallmarks of nonequilibrium behavior, i.e. the nonlocal character of the large deviation functions controlling the statistics of fluctuations out of equilibrium [67]. Such nonlocality, which contrasts with equilibrium phenomenology, emerges from tiny $\mathcal{O}\left(N^{-1}\right)$ 

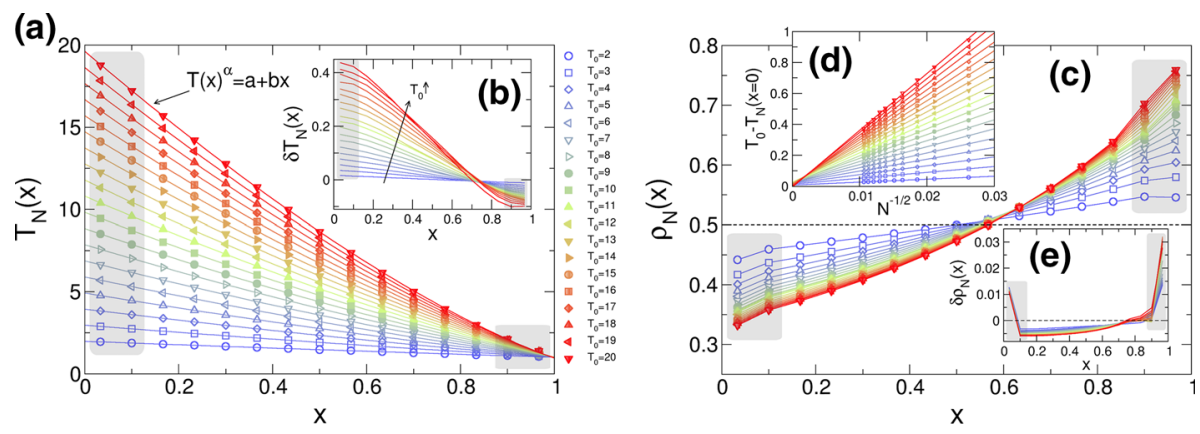

Fig. 4 a Temperature profiles along the gradient direction for $N=8838, \eta=0.5$ and varying $T_{0} \in[2,20]$. Lines are nonlinear fits of the form $T(x)^{\alpha}=a x+b$ [73]. Shaded (light grey) areas correspond to boundary layers (the first two cell columns adjacent to each wall) where the wall defect alters measurements. b Finitesize corrections as described by $\delta T_{N}(x) \equiv T_{N_{\max }}(x)-T_{N_{\min }}(x)$, with $N_{\max }=8838$ and $N_{\min }=1456$, for different gradients. c Density profiles for the same conditions than panel a. d Thermal boundary resistance, see main text, as a function of $N^{-1 / 2}$ for different values of $T_{0}$, together with linear fits. e Finite-size effects in density profiles, as captured by $\delta \rho_{N}(x)$, localize near the thermal walls

corrections to LTE which spread over macroscopic regions of size $\mathcal{O}(N)$, being $N$ the number of particles in the system of interest [68-71]. This shows that LTE is a subtle property: while corrections to LTE vanish locally in the $N \rightarrow \infty$ limit, they have a fundamental impact on nonequilibrium large deviation functions in the form of nonlocality, which in turn gives rise to the ubiquitous long-range correlations which characterize nonequilibrium fluids $[5,62,72]$.

In this section we want to probe the local equilibrium property in the hard disks fluid under a temperature gradient in the absence of gravity. In order to characterize the inhomogeneous nonequilibrium steady state in the fluid, we divide the system into $n_{c}=15$ virtual cells of linear size $\Delta=1 / 15$ along the (gradient) $x$-direction. We measure locally, following the method explaind in Sect. 3, a number of relevant observables including the local average kinetic energy, packing fraction, virial pressure, etc., as well as the energy current flowing through the thermal baths and the pressure exerted on the walls. This last observable will be compared with the bulk-averaged virial pressure to further guarantee the validity of our measurements. Moreover, the local temperature is defined via equipartition theorem from the average kinetic energy per particle in each local cell.

Figure $4 \mathrm{a}$, c shows the temperature and density profiles, $T(x)$ and $\rho(x)$ respectively, measured for the largest system size $N=8838$, a moderate global packing fraction $\eta=0.5$, and varying gradients $\Delta T$. The resulting profiles are typically nonlinear, as expected from Fourier's law with a temperature- and density-dependent heat conductivity (see e.g. Eq. (35) in the Enskog approximation). Note that similar profiles are obtained for different $N, \eta$ and $\Delta T$. As an interesting side comment, note that temperature profiles in Fig. 4a can be fitted with high accuracy by the phenomenological law

$$
T(x)^{\alpha}=a x+b
$$

with $\alpha$ an empirical exponent characterizing the apparent nonlinearity. This simple law has been deduced for some two-dimensional Hamiltonian and stochastic models of heat transport [73], though its microscopic validity for the hard disks fluid is unclear. Note that, in our case, the fitted exponent $\alpha$ exhibits a pronounced dependence on $N$ and $\Delta T$ (not shown), with $\alpha \in[0.681,0.715]$ and no coherent asymptotic behavior, a trait of the strong finite size 

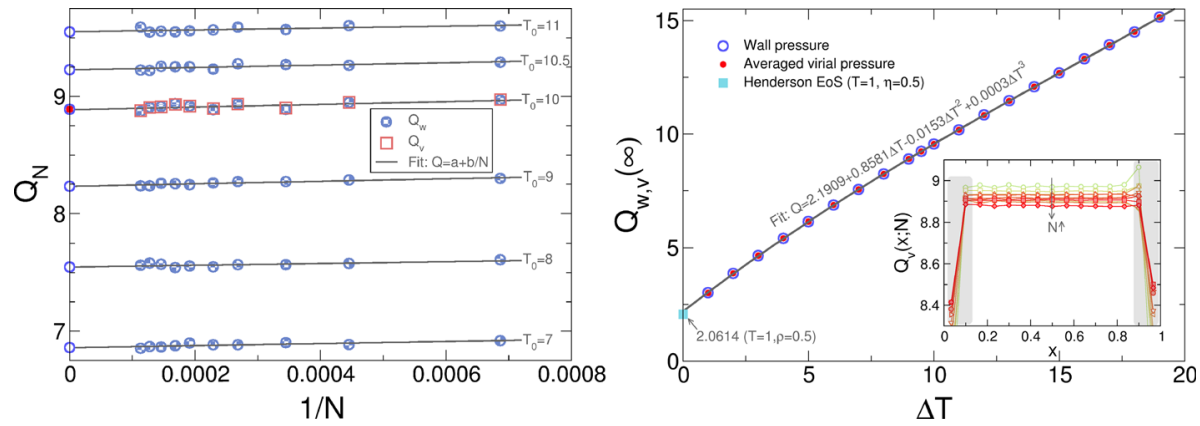

Fig. 5 Left panel: Finite-size analysis of wall reduced pressure $Q_{w}$ for different temperature gradients. The measured $Q_{w}$ (filled circle) decays linearly with $N^{-1}$ for each $T_{0}$. Solid lines are linear fits to the data, and the extrapolation to $N \rightarrow \infty$ is shown as open circles (open circle). The measured bulk-averaged virial reduced pressure $Q_{v}$ is also shown for $T_{0}=10$ (open square), as well as its $N \rightarrow \infty$ extrapolation. Right panel: Values of $Q_{w}$ and $Q_{v}$ extrapolated to $N \rightarrow \infty$ as a function of $\Delta T=T_{0}-T_{L}$ for $\eta=0.5$, together with cubic fit to the data. The light blue squared symbol (filled square) at $\Delta T=0$ represents the equilibrium pressure predicted by the Henderson 77 EoS, see Eq. (9). The inset shows virial reduced pressure profiles for different $N, \eta=0.5$ and $\Delta T=10$. These profiles are constant in the bulk but exhibit strong boundary corrections due to the presence of the walls (Color figure online)

corrections affecting the hydrodynamic profiles. These corrections are captured for instance by the finite-size excess density and temperature profiles, defined as

$$
\delta f_{N}(x) \equiv f_{N_{\max }}(x)-f_{N_{\min }}(x),
$$

with $f \equiv \rho, T$, and $N_{\max }=8838$ and $N_{\min }=1456$ the maximum and minimum number of particles used in these simulations. Figure $4 \mathrm{~b}$, d show both $\delta T_{N}(x)$ and $\delta \rho_{N}(x)$, respectively, measured for different temperature gradients. These observables pinpoint the importance of finite-size corrections in this setting, particularly near the boundaries and most evident for density profiles. In this respect, note that the thermal walls act as defect lines disrupting the local structure of the surrounding fluid, a perturbation that spreads for a finite penetration length toward the bulk fluid. This defines two boundary layers where finite size effects and boundary corrections sum up, see e.g. shaded areas in Fig. 4b, e, and the inset to the right panel in Fig. 5 below.

In addition, the boundary disturbance gives rise to a thermal resistance or temperature gap between the profile extrapolated to the walls, $T(x=0, L)$, and the associated bath temperature $T_{0, L}$. This thermal resistance is a well-known finite-size effect described in literature [74]. We analyze the system size dependence of this thermal gap, $\gamma_{0, L}(\Delta T, N) \equiv$ $\left|T_{0, L}-T(x=0, L)\right|$, in Fig. 4d, finding that

$$
\gamma_{0, L}(\Delta T, N) \sim N^{-1 / 2} \forall \Delta T .
$$

This shows that boundary thermal gaps for hard disks in $2 d$ disappear according to a power law in the thermodynamic limit when approached at constant packing fraction $\eta$ and temperature gradient $\Delta T$. Equivalently to what was done in the equilibrium simulations of Sect. 4, and in order to minimize boundary corrections, we hence proceed to eliminate from our analysis below the boundary layers by removing from the measured profiles the two cells immediately adjacent to each wall, i.e. cells $i=1,2$ and 14, 15 (see shaded areas in Fig. 4) [75].

We next focus on the reduced pressure $Q \equiv \pi \ell^{2} P$ (with $P$ the pressure), that we measure using two different methods. First we measure the reduced pressure exerted by the fluid on the thermal walls, $Q_{w}(N)$, in terms of the average momentum exchanged between the 
colliding particles and the thermal wall per unit length and unit time. ${ }^{2}$ As done in Sect. 4, we also measure the local virial reduced pressure $Q_{v}(x ; N)$ using the definition of Eq. (42) for each virtual column of the fluid. This method yields pressure profiles which are constant across the bulk of the fluid but exhibit clear structure at the boundary layers, see inset in right panel of Fig. 5. This is of course expected because of the local anisotropy induced by the nearby walls. A sound definition of the fluid's pressure is then obtained by averaging in the bulk the virial pressure profiles. Both the wall and bulk-averaged virial pressures exhibit mild finite-size corrections which scale linearly with $N^{-1}$ for each $\Delta T$, see left panel and right inset in Fig. 5, converging to a well-defined value in the $N \rightarrow \infty$ limit. In all cases (both for finite $N$ and in the $N \rightarrow \infty$ limit) the measured wall and virial values agree to a high degree of accuracy $\forall \Delta T$, see right panel in Fig. 5. The asymptotic $N \rightarrow \infty$ data are consistent with a cubic polynomial dependence of pressure on $\Delta T$, see right panel in Fig. 5, an empirical law which is in turn compatible with the equilibrium $(\Delta T=0)$ pressure predicted by the improved Henderson EoS [9,76]

$$
\bar{Q}_{\mathrm{H} 77}(\rho)=\frac{1+\rho^{2} / 8}{(1-\rho)^{2}}-0.043 \frac{\rho^{4}}{(1-\rho)^{3}} .
$$

This is just the original Henderson equation of state, see Eq. (9) above, corrected with some weak higher-order term to improve empirical accuracy against moderate-density data $[9,76]$. We hence can conclude that both wall and virial definitions of pressure are compatible with each other in arbitrary quiescent nonequilibrium steady states (and compatible with limiting equilibrium predictions), suggesting already a sort of local mechanical equilibrium in the nonequilibrium fluid.

Macroscopic LTE [75] implies that, locally in a nonequilibrium steady state, the density and temperature fields should be related via the equilibrium equation of state,

$$
Q=\rho T \bar{Q}(\rho, T)
$$

where $\bar{Q}(\rho, T)$ is the so-called compressibility factor [9]. In order to probe this property, we plot in the left panel of Fig. 6 values for $\bar{Q} \equiv Q /[T(x) \rho(x)]$ measured out of equilibrium, as a function of $\rho(x)$ and $T(x)$. Note that each nonequilibrium simulation, for fixed $(\Delta T, \eta, N)$, covers a fraction of the EoS surface, thus improving the sampling when compared to equilibrium simulations, which yield a single point on this surface. As shown in Sect. 3, hard disks exhibit density-temperature separability, meaning that temperature scales out of all thermodynamic relations [9,33], so the associated $\bar{Q}$ depends exclusively on density. In this way we expect a complete collapse for the projection of the EoS surface on the $\bar{Q}-\rho$ plane, as we indeed observe, see left panel in Fig. 6.

Another interesting property concerns the finite-size corrections to the measured EoS. Strikingly, although density and temperature profiles, as well as pressures, all depend strongly on $N$, see Figs. 4 and 5, the measured $\bar{Q}_{N}$ as a function of the local density exhibits no finite size corrections at all. This is better shown in the right panel of Fig. 6, where a total of 2530 data points for different $N \in[1456,8838], \eta \in[0.05,0.65]$ and $T_{0} \in[2,20]$ are shown. This lack of finite-zise corrections for the EoS strongly suggests a compelling structural decoupling between the bulk fluid, which behaves macroscopically and thus obeys locally the thermodynamic EoS, and the boundary layers near the thermal walls, which sum up all sorts of artificial finite-size and boundary corrections to renormalize the effective boundary conditions

\footnotetext{
2 Note that this method would have proven useless in the equilibrium simulations of Sect. 4 (except for $g=0$ ), as the fluid pressure under a gavitational field exhibits a nontrivial spatial structure, as dictated by the barometric formulas (39)-(40).
} 

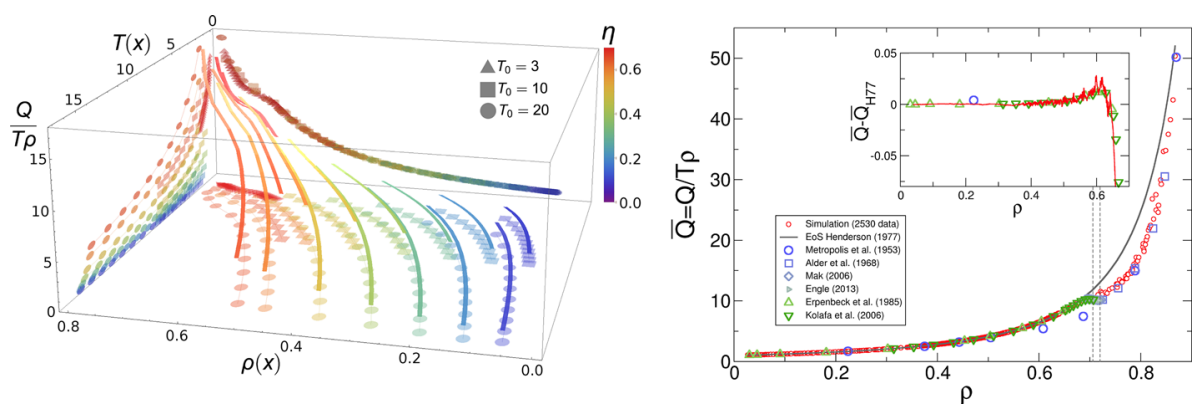

Fig. 6 Left panel: Compressibility factor $\bar{Q} \equiv Q /[T(x) \rho(x)]$ as a function of $T(x)$ and $\rho(x)$ measured for $N=2900$ hard disks and different global packing fractions $\eta$ and boundary temperatures $T_{0}$. Right panel: $\bar{Q}$ vs $\rho(x)$ measured for $\eta=0.5, N \in[1456,8838]$ and $T_{0} \in[2,20]$, as well as for $\eta \in[0.05,0.65], N=2900$ and $T_{0}=10,20$, summing up a total of 2530 data points. For comparison, data from previous equilibrium simulations in literature are shown, together with the Henderson $77 \mathrm{EoS}$ approximation (solid line). The inset shows a detailed comparison of a running average of our data with equilibrium results after subtracting the leading Henderson 77 behavior

on the remaining bulk. This remarkable bulk-boundary decoupling phenomenon is even more surprising at the light of the long range correlations generically present in nonequilibrium fluids [5,62,72], offering a tantalizing computational method to obtain macroscopic properties of nonequilibrium fluids without resorting to unreliable finite-size scaling extrapolations $[33,39]$. We will show in next section how this bulk-boundary decoupling phenomenon allows to obtain some universal master curves which characterize transport in the hard disk fluid, as well as to characterize the macroscopic conductivity functional and its marginal anomaly in $2 d$ [33].

For comparison, the right panel of Fig. 6 also includes several data sets from a number extensive equilibrium simulations carried with different methods during the last 60 years [44,77-82], as well as the improved Henderson EoS prediction, see Eq. (46) above, which is reasonably accurate in the fluid phase. The inset in this figure shows a detailed comparison of a running average of our extesive data and the equilibrium simulations in literature, once the leading Henderson' 77 behavior [76] has been subtracted. The excellent agreement (to within $1 \%$ relative error) confirms the validity and robustness of the macroscopic LTE property and the bulk-boundary decoupling phenomenon here described. Note also that the accuracy of our data for the EoS is surprising taking into account that local cells have at most 500 particles, and many fewer in the typical case. We again trace back this robust self-averaging behavior to the strong chaotic behavior of the hard disks system, see Sect. 4 above.

We also stress that some of our data sets for high enough global packing fractions include points across and beyond the controversial liquid-hexatic-solid double phase transition regime $[44,77]$. In fact, we find in our simulations that for $\eta \gtrsim 0.6$ a coexistence between a fluid phase near the hot wall and a solid-like phase near the cold one is established, very much in the spirit of the stationary Stefan problem [83]. Figure 7 shows a typical configuration in this nonequilibrium coexistence regime with two color codings. The right one represents the local hexatic order parameter $\psi_{6}[44,77]$, with

$$
\psi_{6} \equiv \frac{1}{N_{b}} \sum_{k=1}^{N_{b}} \mathrm{e}^{\mathrm{i} \phi_{k}}
$$




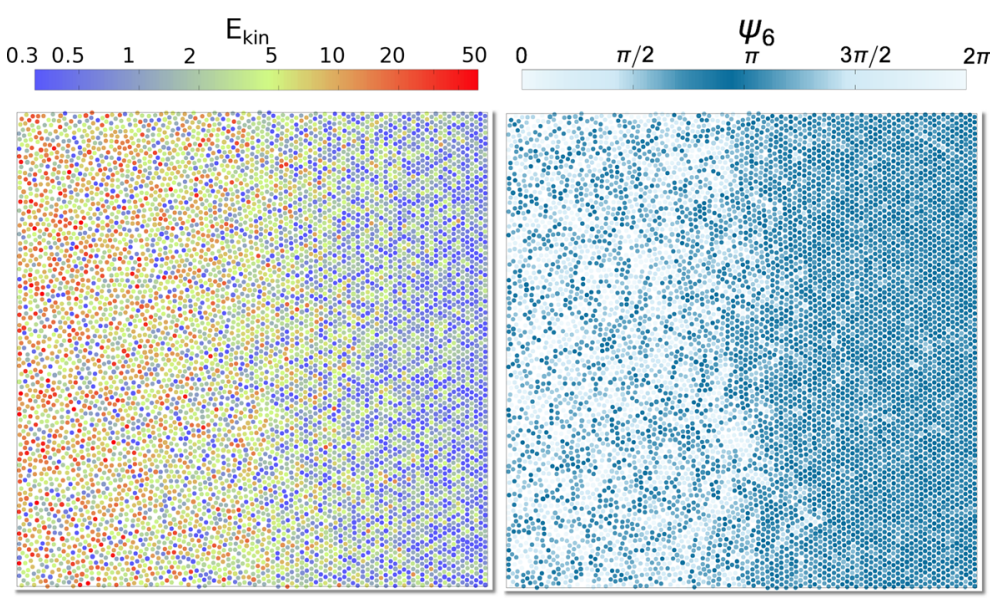

Fig. 7 Snapshot of a typical dense configuration of $N=7838$ hard disks under a temperature gradient. In particular the global packing fraction here is $\eta=0.7$, and the left and right boundary temperatures are $T_{0}=10$ and $T_{L}=1$, respectively. The figure displays the same configuration with two different color codes, representing respectively the the kinetic energy (left) and local hexatic order $\psi_{6}$ (right), see definition in the main text. Inhomogeneous fluid and solid phases coexist for such high $\eta$ under a temperature gradient, with a rough interface separating both regions (Color figure online)

where $N_{b}$ is the number of nearest neighbors of a given disk and $\phi_{k}$ is the angle of the bond connecting the reference disk with its $k$-neighbor, relative to an arbitrary direction $(\hat{x}$ in our particular case). Interestingly $\psi_{6}$ picks the local hexatic order of the symmetry-broken phase, offering an valuable method to characterize the interface between the inhomogeneous fluid and solid phases. This coexistence appears in the presence of a strong temperature gradient and an associated heat current, as captured by the second color code which represents kinetic energy in the left panel of Fig. 7. This interesting nonequilibrium fluid/solid coexistence can be confronted against recent theoretical predictions [83-86].

To end this section, some words on microscopic local thermodynamic equilibrium are in order. Indeed, the macroscopic notion of LTE that we have just confirmed in simulations does not carry over to microscopic scales. The local statistics associated to a fluid's mNESS is typically more complicated than the LTE local Gibbs measure, containing small (but intricate) corrections which are essential for transport to happen. In order to detect these corrections, and pursuing the analogy with stochastic lattice gases [60,68-71], we also studied the $n$-th order central moments $m_{n}(u) \equiv\left\langle(u-\langle u\rangle)^{n}\right\rangle$ of a global observable, the total energy per particle $u$ of the hard disks fluid, with

$$
u \equiv \frac{1}{N} \sum_{i=1}^{N} \frac{1}{2} m \mathbf{v}_{i}^{2} .
$$

Figure 8a-c show the measured $m_{n}(u), n=1,2,3$, as a function of temperature gradient $\Delta T$ for global packing fraction $\eta=0.5$ and different $N$, together with the $N \rightarrow \infty$ extrapolation of the measured data and the LTE estimates for energy moments, $m_{n}(u)^{\text {le }}$ (see Appendix C of Ref. [32] for a detailed derivation of these LTE expressions). Interestingly, we observe that while the average energy does indeed follow the LTE behavior, $\langle u\rangle \sim\langle u\rangle^{\mathrm{le}}$, energy fluctuations (as captured e.g. by the second central moment $m_{2}(u)$ in Fig. 8b) exhibit increasing deviations from the LTE estimate. Remarkably, the excess energy fluctuations exhibit a linear scaling with the squared gradient, 


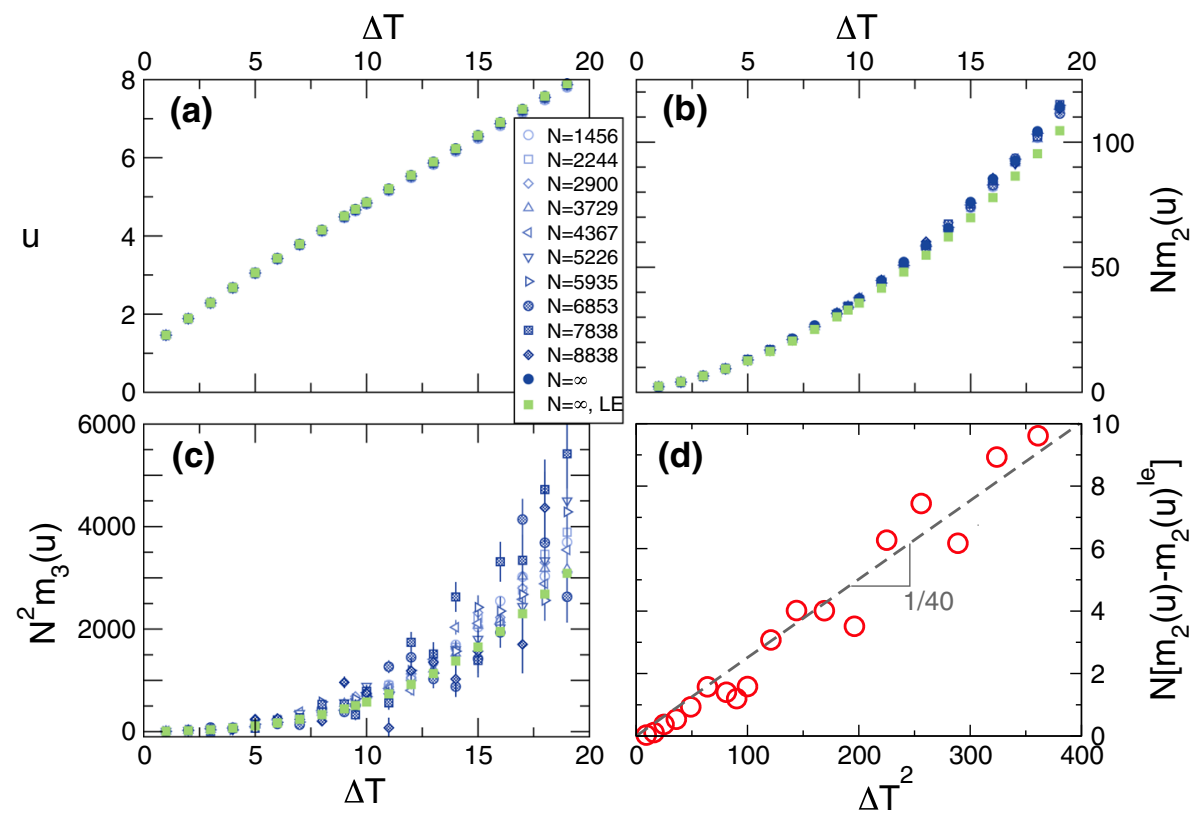

Fig. 8 Panels a-c show the scaled central moments of the total energy of the hard disks fluid, $m_{n}(u) \equiv$ $\left\langle(u-\langle u\rangle)^{n}\right\rangle$, with $u \equiv N^{-1} \sum_{i=1}^{N} m \mathbf{v}_{i}^{2} / 2$, as a function of temperature gradient $\Delta T$ for a global packing fraction $\eta=0.5$ and varying $N$. Filled symbols correspond to the $N \rightarrow \infty$ limit of our data (filled circle) and after assuming LTE (filled square). d The excess energy variance (as compared to LTE) scales linearly with $\Delta T^{2}$ with a slope $\approx 1 / 40$

$$
\delta m_{2}(u) \equiv N\left[m_{2}(u)-m_{2}(u)^{\mathrm{le}}\right] \approx+\frac{1}{40} \Delta T^{2},
$$

see Fig. 8d, a result strongly reminiscent of the behavior observed in schematic models like the Kipnis-Marchioro-Presutti (KMP) model of heat transport or the symmetric exclusion process (SEP) [68-71], where $\delta m_{2}(u)= \pm \Delta T^{2} / 12$. In this way, energy fluctuations for hard disks are enhanced with respect to LTE, $\delta m_{2}(u)>0$, as happens for the KMP model and contrary to the observation for SEP [68-71], although the excess amplitude is roughly three times smaller for disks. We also mention that a similar study (not shown, see [32]) of the global velocity moments, $v_{n} \equiv\left\langle N^{-1} \sum_{i=1}^{N}\left|\mathbf{v}_{i}\right|^{n}\right\rangle$, shows no deviations from the LTE prediction for these observables. The reason why energy fluctuations do pick up corrections to LTE while velocity fluctuations exhibit LTE behavior lies in the nonlocal character of energy fluctuations. Indeed, while $v_{2}$ includes only a sum of local factors, $m_{2}(u)$ includes nonlocal contributions of the form $\left\langle\mathbf{v}_{i}^{2} \mathbf{v}_{j}^{2}\right\rangle, i \neq j$, summed over the whole system. In this way small $\mathcal{O}\left(N^{-1}\right)$ corrections to LTE extending over large $\mathcal{O}(N)$ regions give rise to weak long-range correlations in the system which, when summed over macroscopic regions, yield a net contribution to energy fluctuations, which thus depart from the LTE expectation [6871]. The observed breakdown of LTE at the energy fluctuation level is hence a reflection of the nonlocality of the underlying large deviation function governing fluctuations in the nonequilibrium fluid. This result is, to our knowledge, the first evidence of a nonlocal large deviation function in a realistic model fluid. 


\section{Scaling in Fourier's Law}

A main feature of a typical nonequilibrium steady state is the nontrivial, inhomogeneous spatial structure that the system of interest develops in response to the nonequilibrium driving, see e.g. Fig. 4 above. This structure, readily measurable in experiments or simulations, corresponds to the solution of the associated Navier-Stokes equations, see Sect. 3, and carries information on the governing nonequilibrium macroscopic laws (e.g. Fourier's law) which emerge from the myriad of interacting microscopic constituents. A key goal of statistical physics is therefore to understand general properties of these spatial structures, consubstantial to nonequilibrium behavior. With this idea in mind, in this section we derive a set of simple yet general scaling laws for a broad class of $d$-dimensional fluids driven far from equilibrium by a temperature gradient in the absence of gravity [33], and we test them in hard disks simulations. In particular, we will show that the fluid's density and temperature profiles follow from two master curves, independent of the driving force and the system parameters, after a simple linear scaling of space in terms of the flowing heat current.

This result is based on two hypotheses on the macroscopic behavior of the nonequilibrium fluid. The first one is macroscopic LTE, a property that as we have demonstrated in the previous section holds robustly for the hard disks fluid arbitrarily far from equilibrium. In fact we used this property in Sect. 5 to provide an accurate estimate of the hard disks EoS, see Fig. 6. The second hypothesis concerns the macroscopic transport properties of the fluid, that should be described by Fourier's law. In particular this law states that the steady-state heat current $J$ in a driven system is proportional to the applied boundary temperature gradient $[8,21-26,39,74,87-90]$, i.e.

$$
J=-\kappa(\rho, T) \frac{d T(x)}{d x}, \quad x \in[0,1]
$$

where $\kappa(\rho, T)$ is the thermal conductivity, that may depend in general on the local temperature $T(x)$ and on the local packing fraction $\rho(x)$. Fourier's law (51) is usually expected to hold for macroscopic systems in the limit of small temperature gradients, while higher-order (Burnett) corrections in the gradient are typically conjectured for stronger driving [9]. However, our results below will prove that, at least for quiescent heat transfer, these corrections are absorbed into a nonlinear conductivity functional, extending the validity of Fourier's law deep into the strongly nonlinear regime.

Interestingly, the assumption of macroscopic LTE allows us now to write Fourier's law in terms only of the density field. To do so, we need the EoS to be invertible in the $(\rho, T)$ range of interest, an assumption which holds valid for most fluids away from a critical point. In this case, inverting the $\operatorname{EoS} Q=q(\rho, T)$ yields $T=f_{Q}(\rho)$, with $f_{Q}(\rho)$ an uniparametric curve such that $q\left[\rho, f_{Q}(\rho)\right]=Q$. Similarly, the heat conductivity follows as $\kappa(\rho, T)=\kappa\left[\rho, f_{Q}(\rho)\right] \equiv k_{Q}(\rho)$, defining another uniparametric function $k_{Q}(\rho)$. In this way, Fourier's law (51) can be rewritten as

$$
J=G_{Q}^{\prime}(\rho) \frac{d \rho}{d x}=\frac{d G_{Q}(\rho)}{d x},
$$

where $G_{Q}^{\prime}(\rho) \equiv-k_{Q}(\rho) f_{Q}^{\prime}(\rho)$ and 'denotes derivative with respect to the argument. Note that to write the transport problem in terms of the density field $\rho(x)$, we also need to provide boundary conditions for the latter. These boundary conditions, $\rho(0)=\rho_{0}$ and $\rho(1)=\rho_{L}$, can be inferred from the constraints 


$$
\frac{T_{0}}{T_{L}}=\frac{f_{Q}\left(\rho_{0}\right)}{f_{Q}\left(\rho_{L}\right)}, \quad \eta=\int_{0}^{1} \rho(x) d x=\frac{\int_{\rho_{0}}^{\rho_{L}} \rho G_{Q}^{\prime}(\rho) d \rho}{G_{Q}\left(\rho_{L}\right)-G_{Q}\left(\rho_{0}\right)} .
$$

These equations, together with Eq. (52), completely define the macroscopic transport problem in terms of $\rho(x)$. The reduced pressure and the heat current can be now obtained as $Q=$ $q\left(\rho_{0}, T_{0}\right)$ and $J=\left[G_{Q}\left(\rho_{L}\right)-G_{Q}\left(\rho_{0}\right)\right]$. A striking consequence of our simple macroscopic hypotheses can be now directly deduced from Eq. (52). Indeed, as both $J$ and $Q$ are statedependent constants in the absence of gravity, Eq. (52) immediately implies that $G_{Q}[\rho(x)]=$ $J x+\zeta$, i.e. $G_{Q}[\rho(x)]$ is a linear function of position, with slope $J$ and $\zeta=G_{Q}\left(\rho_{0}\right)$ an arbitrary constant, or equivalently ${ }^{3}$

$$
\rho(x)=G_{Q}^{-1}(J x+\zeta) .
$$

Therefore, we conclude that there exists a single master surface $\bar{\rho}_{Q}(z) \equiv G_{Q}^{-1}(z)$ in $z-Q$ space from which any steady state density profile follows after a linear spatial scaling $x=$ $(z-\zeta) / J$. Furthermore, this scaling behavior is immediately inherited by temperature profiles via the local EoS, which yields another master surface $\bar{T}_{Q}(z)=f_{Q}\left[G_{Q}^{-1}(z)\right]$. These simple yet general scaling laws completely characterize heat flow in the system of interest, and are independent of the global packing fraction $\eta$ or the nonequilibrium driving defined by the baths temperatures $T_{0}$ and $T_{L}$, depending exclusively on the uniparametric functions $f_{Q}(\rho)$ and $k_{Q}(\rho)$ controlling the system macroscopic behavior. Remarkably, Eq. (54) also implies that any measured steady density and temperature profiles can be collapsed onto the master surfaces $\bar{\rho}_{Q}(z)$ and $\bar{T}_{Q}(z)$, respectively, by scaling space by the associated current $J$ and shifting the resulting profile an arbitrary constant $\zeta$. This suggests a simple scaling method to obtain the master curves in simulations and experiments that we exploit below.

For hard disks systems, as well as for any other system with homogeneous interparticle potential $V(\mathbf{r}) \propto r^{-n}$, both the EoS and the heat conductivity exhibit a well-known densitytemperature separability $[33,91]$, a property that simplifies the form of the general scaling laws derived above. In this case the EoS takes the separable form $Q=T q(\rho)$, with $q(\rho)$ a typically unknown function (for which accurate approximations exist in the case of hard disks, see e.g. Henderson formulas, Eqs. (9) or (46) $[9,92])$. The conductivity also takes the separable form $\kappa(\rho, T)=\sqrt{T} k(\rho)$, where again $k(\rho)$ is generally unknown (though a reasonably good approximation is obtained from Enskog kinetic theory [34,93], see Eq. (35)). It is then easy to show that, in this simpler case, the master surfaces $\bar{\rho}_{Q}(z)$ and $\bar{T}_{Q}(z)$ boil down to a pair of universal curves. In particular, for hard disks

$$
G_{Q}(\rho)=Q^{3 / 2} G(\rho), \quad \text { with } G^{\prime}(\rho) \equiv k(\rho) q(\rho)^{-5 / 2} q^{\prime}(\rho),
$$

so all density profiles scale as $\rho(x)=G^{-1}(\psi x+\zeta)$, with $\psi \equiv J / Q^{3 / 2}$ the reduced current and $\zeta=G\left(\rho_{0}\right)$. This defines a master curve $\bar{\rho}(z)=G^{-1}(z)$ from which all density profiles follow after scaling space as $x=(z-\zeta) / \psi$, irrespective of the driving gradient or the global density. Moreover, temperature profiles scale now as $T(x) / Q=q[\rho(x)]^{-1}$, defining another master curve $\bar{T}(z)=q[\bar{\rho}(z)]^{-1}$. Note that similar scaling laws hold for any $d$-dimensional fluid with homogeneous interactions (including hard hyperspheres) [33].

Of course, the scaling functions $\bar{\rho}(z)$ and $\bar{T}(z)$ are unknown because we lack exact expressions for the density dependence of both the hard disks EoS and conductivity. However, we can obtain these master curves from the simulation data presented in Sect. 5, see Fig. 4,

\footnotetext{
$\overline{3}$ Note that we assume here that $G_{Q}(\rho)$ has a well-defined inverse $G_{Q}^{-1}(z)$. This assumption seems reasonable as steady density profiles are typically well behaved.
} 
Fig. 9 a Bulk density profiles for $N=2900$ hard disks, $T_{0}=20$ and varying $\eta \in[0.15,0.65]$, as a function of $\psi x=J x / Q^{3 / 2}$. By shifting each curve an amount $\zeta$, a perfect collapse is obtained which reconstructs the master curve $\bar{\rho}(z)$. b Measured reduced currents $\psi$ and shifts $\zeta$ as a function of $\Delta T$ for different $N$ and global packing fraction $\eta=0.5$. Finite size effects are apparent for both magnitudes

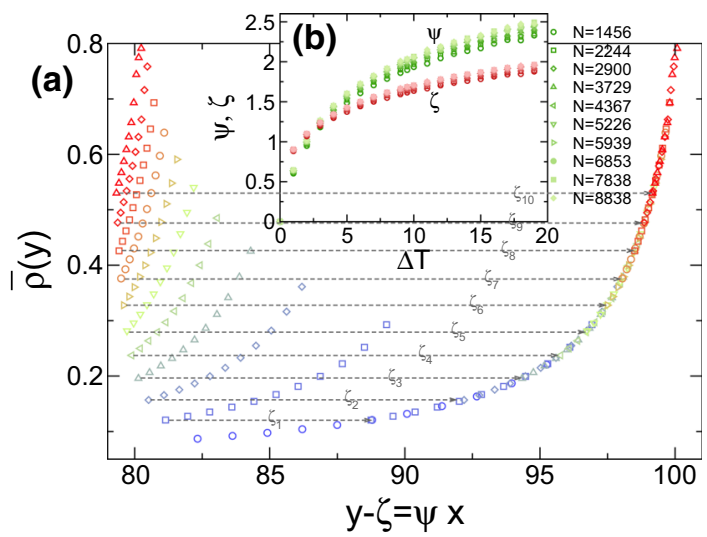

(a)

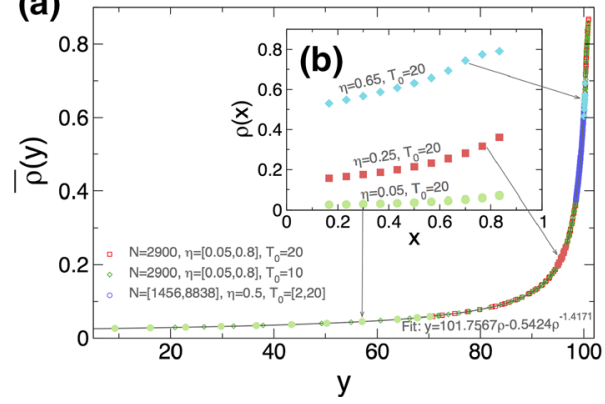

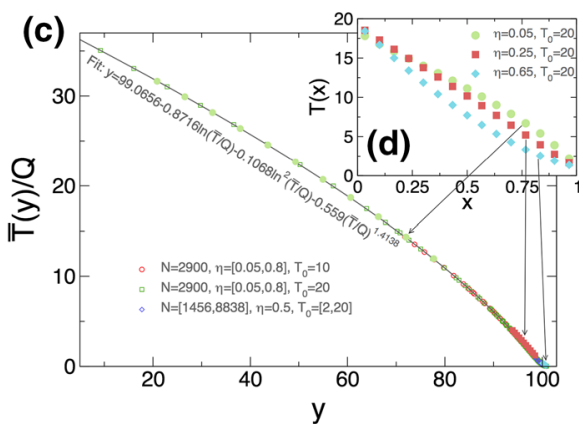

Fig. 10 a Collapse of scaled bulk density profiles measured for $N \in[1456,8838]$ and different sets of conditions (see legend) for a total of more than 4000 data points. b Widely different bulk density profiles measured for different conditions collapse onto different parts of the same master curve. $\mathbf{c}, \mathbf{d}$ Collapse of bulk temperature profiles for the same conditions that the top panel. Note that the shifts $\zeta$ obtained from the density scaling yield a perfect scaling for temperature profiles

using the previous scaling scheme. To do so, we first proceed to eliminate from the measured hydrodynamic profiles the boundary layers described in Sect. 5, where finite size effects and boundary corrections concentrate. In particular, we remove from the profiles the two cells immediately adjacent to each wall (see shaded areas in Fig. 4a, d. The bulk density profiles $\rho(x)$ so obtained are then scaled using the reduced current $\psi=J / Q^{3 / 2}$ in each case, that we calculate by measuring the finite-size heat current $J$ and reduced pressure $Q$, and shifted by a constant $\zeta$ to achieve a maximum overlap among all scaled profiles. Figure 9a shows an example of this scaling procedure for density profiles (see also [94] for a multimedia explanation of the scaling procedure).

Using this scaling method we were able to collapse onto a single master curve $\bar{\rho}(z)$ a large amount of data for density profiles measured for different sizes $N$, boundary gradients $\Delta T$ and global densities $\eta$, see Fig. 10a. Moreover, using the same shifts $\zeta$ obtained from the collapse of density profiles, the rescaled temperature profiles also collapsed onto another master curve $\bar{T}(z)$, see Fig. 10c. Strikingly, while the measured $J, Q, \psi$ and $\zeta$ all depend appreciably on $N$ for each $\Delta T$ and $\eta$ (see the inset Fig. 10b), the collapsed data show no appreciable finitesize corections, defining two master curves as predicted by the macroscopic theory. Such remarkable collapse hence implies that the measured bulk hydrodynamic profiles are those of a macroscopic hard disks fluid obeying Fourier's law and subject to some renormalized, 
effective boundary conditions set by the boundary layers, which sum up all sorts of finitesize effects and boundary corrections. This is another instance of the striking bulk-boundary decoupling phenomenon already described in Sect. 5. It implies a fine structural self-tuning of the fluid, which goes beyond the mere presence of boundary layers, and is even more surprising at the light of the long range correlations present in nonequilibrium fluids [72,95]. This bulk-boundary decoupling phenomenon is likely to appear in other complex systems driven out of equilibrium by different boundary reservoirs, and offers a tantalizing scaling method to avoid unreliable finite-size scaling extrapolations. In fact, a standard finite-size scaling analysis of our data, aimed at obtaining first the asymptotic $(N \rightarrow \infty)$ observables $\rho_{\infty}(x), J_{\infty}$ and $Q_{\infty}$ for each $\Delta T$ and $\eta$ to perform then the scaling collapse, fails badly as none of these observables follow a well-defined asymptotic behavior.

It is interesting to note also that the excellent scaling behavior of our data strongly suggests that, quite remarkably, Fourier's law (51) remains empirically valid even under strong temperature gradients, extending its range of validity deep into the highly nonlinear regime. This means in particular that the higher order (Burnett) corrections conjectured for strong driving are in this case absorbed into the nonlinear conductivity $\kappa(\rho, T)$ in Eq. (51). ${ }^{4}$ The combination of our scaling analysis and the bulk-boundary decoupling phenomenon here described hence allows to obtain clean properties of macroscopic nonequilibrium fluids from finite-size numerical simulations or experiments. The two master curves in Fig. 10 have full predictive power, as we can deduce from them and the scaling formulas in Eqs. (52)-(54) the density and temperature profiles of a macroscopic hard disk system for any set of parameters $T_{0}, T_{L}$ and $\eta$.

Our determination of the master curves in Fig. 10 is so detailed that it allows for a precise measurement of the hard disks heat conductivity over a broad range of packing fractions. In fact, by multiplying Fourier's law (51) by $Q^{-3 / 2}$ and recalling the separable form of the conductivity, $\kappa(\rho, T)=\sqrt{T} k(\rho)$, one can easily show that

$$
k(\rho)=\frac{1}{\sqrt{\bar{T}(z)}\left|\bar{T}^{\prime}(z)\right|}=\frac{J}{\sqrt{T(x)}\left|T^{\prime}(x)\right|},
$$

with $\rho=\bar{\rho}(z)$. We hence performed discrete derivatives of the measured master curve $\bar{T}(z)$ (see Fig. 10c) for each of the different sets of parameters $\Delta T, \eta$ and $N$, identifying each value of $\left[\sqrt{\bar{T}(z)} \bar{T}^{\prime}(z)\right]^{-1}$ with the associated $\rho=\bar{\rho}(z)$. Figure 11a shows the so obtained $k(\rho)$, which exhibits weak but apparent deviations from the Gass prediction [34] based on Enskog kinetic theory, as already reported in previous simulations [93,98]. In addition, a very weak but systematic $\sqrt{\ln N}$-dependence of $k(\rho)$ is observed, see Fig. 11b. This observation confirms for the first time (to our knowledge) and with high accuracy the marginally, $\sim \sqrt{\ln N}$ anomalous heat conductivity predicted for hard disks as a result of the long time tails in two dimensions [17-19]. This result demonstrates that the scaling method here presented, together with the bulk-boundary decoupling phenomenon, allows one to investigate transport numerically in a more rigorous way, getting rid of artificial finite-size effects in simulations related with the presence of boundaries, which result in systematic errors in heat conductivity measurements, while keeping physically relevant bulk finite-size information.

We now turn our attention to the effect of gravity on transport and the subsequent transition to convective flow observed in hard disks [40].

\footnotetext{
${ }^{4}$ Note however that, for more complex flow situations, as e.g. acceleration-driven Poiseuille flow, Burnett corrections seem to play a key role $[96,97]$.
} 


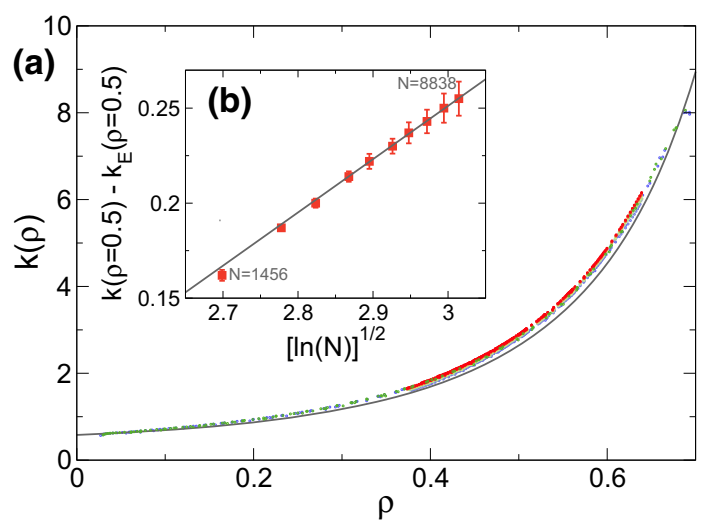

Fig. 11 a Density dependence of the heat conductivity as obtained from the rescaled temperature profiles $\bar{T}(z) \equiv T(z) / Q$ for different $\eta \in[0.05,0.8], T_{0} \in[2,20]$ and $N \in[1456,8838]$. A well-defined deviation from Gass result $k_{\mathrm{E}}(\rho)$ based on Enskog kinetic theory (full line) is found [34,93]. Moreover, a systematic dependence with system size $N$ is also observed, see inset $\mathbf{b}$ for $\eta=0.5$, which scales as $\sqrt{\ln (N)}$ for large enough $N$. This observation confirms the marginal anomaly expected for the heat conductivity in $2 d$ hard disks [17-19]

\section{Role of Gravity and Transition to Convective Flow}

In this section we will switch on the gravity field to explore its role on heat transport in the hard disks fluid. In particular, we will focus on the Bénard problem, which consists in a fluid that is heated from below in the presence of gravity. In this setting, it is empirically observed that a critical value for the temperature gradient exists beyond which convective flow kicks in, while the fluid develops roll structures spanning the whole system. This is the wellknown Rayleigh-Bénard instability, that was first observed experimentally by Bénard and then interpreted theoretically by Rayleigh [99-101]. It is a purely nonequilibrium problem that includes a transition from a conducting to a convective state characterized by a nonzero, structured hydrodynamic velocity field [40].

This is by no means the first study of convective flow in hard particle systems. Indeed, the Bénard problem for hard disks fluids has been studied in the past using computer simulations, see e.g. [102-108]. In particular, Mareschal and coworkers observed numerically the transition to convective flow in systems of hard disks, though the large velocity fluctuations prevented the accurate determination of local magnitudes [102-104,106]. Nevertheless these works managed to measure some hydrodynamics profiles, which exhibited a reasonable agreement with predictions based on the numerical solution of Navier-Stokes equations. Rapaport $[105,107]$ also studied convection in hard disks, demonstrating the strong influence of both boundary conditions and initial states on the convective stationary properties of the hard disk fluid. Finally, let us also mention the work of Risso and Cordero [108] where the onset of the hard-disks convection transition was investigated in detail.

Here we want to characterize the steady state transport properties of the hard disks fluid across the Rayleigh-Bénard instability, and its dependence on the different external parameters, namely the magnitude of the gravity field $g$ and the temperature gradient, controlled by the boundary temperature $T_{0}$. For that we will explore both global observables and local magnitudes. The former include for instance the kinetic energy per particle, heat current, pressure, etc. while the latter will allow us to access the nontrivial spatial structures developed by the hydrodynamic fields beyond the convective instability point: velocity and temperature 


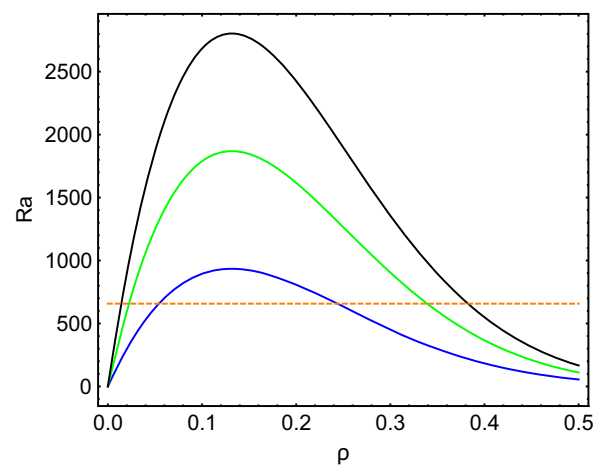

(a)

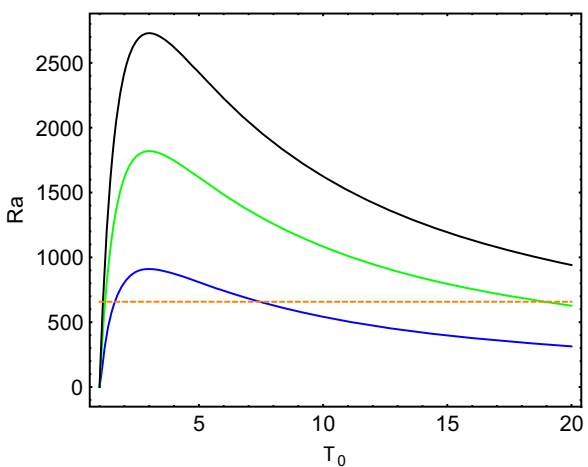

(b)

Fig. 12 Left: Rayleigh number Ra in the Enskog approximation as a function of the global density for $T_{0}=5$, $T_{L}=1$ and $g=5$ (blue curve), $g=10$ (green curve) and $g=15$ (black curve). Dashed orange line is the critical Rayleigh number $\mathrm{Ra}_{c}=27 \pi^{4} / 4 \simeq 657.51$ obtained using Boussinesq approximation on the Navier-Stokes equations [109]. Right: Rayleigh number as a function fo $T_{0}$ for a fixed value of $\eta=0.2$ for the same values of $g$ on the left panel (Color figure online)

fields, streamline structures, etc. Moreover, to study the Bénard problem, we consider now a hard disks system with stochastic thermal walls (see Sect. 2) along the $y$-direction, the same of the external gravity field, with a temperature $T_{0}\left(T_{L}\right)$ at the bottom (top) wall, such that $T_{0}>T_{L}$ so as to favor the instability. We will fix in particular $T_{L}=1$ without loss of generality, see discussion in Sect. 2. In addition, to avoid problems associated to the location of roll structures beyond the critical Bénard gradient, we also consider reflecting boundary walls along the $x$-direction (as opposed to the periodic boundary conditions orthogonal to the gradient directions of the previous sections Sects. 5 and 6).

In order to proceed, we first have to choose the range of external parameters (global density $\eta$, hot bath temperature $T_{0}$ and field strength $g$ ) where the phenomenology of interest emerges. To study the Rayleigh-Bénard instability, it seems reasonable to focus on the Rayleigh number (Ra), an adimensional magnitude whose value changes abruptly between convective and nonconvective flow states. The Rayleigh number is defined as the ratio of the typical timescales for diffusive and convective thermal transport, and can be written as

$$
\mathrm{Ra}=\frac{g \Delta T L^{3}}{\tilde{v} \tilde{\kappa}},
$$

where $\Delta T=T_{0}-T_{L}>0$ measures the external gradient (recall we choose $T_{L}=1$ ), $\tilde{v}=v / \tilde{\rho}$ is the kinematic viscosity with $\tilde{\rho}=m \rho / \pi \ell^{2}$ the mass density, $\tilde{\kappa}=\kappa / \tilde{\rho} C_{P}$, and $C_{P}=c_{p} / m$ is the specific heat capacity per unit mass. For hard disks, linearizing the Navier-Stokes equations under the Boussinesq approximation [109], one arrives to a critical Rayleigh number $\mathrm{Ra}_{c}=27 \pi^{4} / 4 \simeq 657.51$ above which convection kicks in for the stress free boundary condition case. To have some intuition on the range of parameters of interest, we computed Ra using the Henderson EoS approximation (9) and the Enskog transport coefficients for hard disks, Eq. (35), which work pretty well for low and moderate densities, see Fig. 12. The left panel in this figure shows the behavior of Ra as a function of the global density for $T_{0}=5$ and different values of the gravity field, namely $g=5$, 10 and 15. In all cases, the maximum of $\operatorname{Ra}(\eta)$ appears for low densities and it is above the critical Rayleigh number $\mathrm{Ra}_{c}$. It therefore seems convenient to fix the global density to $\eta=0.2$ in our simulations below. In order to gain some insight on the value of the 
critical boundary temperature $T_{0}$ separating non-convective and convective regimes, we plot in the right panel of Fig. $12 \mathrm{Ra}$ as a function of $T_{0}$ for a fixed global density $\eta=0.2$. From these plots we obtain the following critical temperatures as a function of $g$, namely $T_{0}^{c}(g=5)=1.6205, T_{0}^{c}(g=10)=1.2233$ and $T_{0}^{c}(g=15)=1.1376$, indicating that convection appears for lower temperatures as we increase $g$. In this way, our simulations below will focus on the following bottom wall temperatures across the Rayleigh-Bénard instability, $T_{0}=1,1.2,1.4,1.6,1.8,2.0,2.2,2.4,2.6,2.8,3,4, \ldots, 19,20$, with $g=0,5,10$ and 15, and $\eta=0.2$ as mentioned above. Finally, we choose a relatively small number of particles, $N=957$, in order to have a fast system evolution so as to gather a large amount of data to perform averages. We expect that the bulk-boundary decoupling phenomenon reported in previous sections (which enforces the macroscopic laws in the bulk of a finite-sized fluid under renormalized boundary conditions) will allow us to obtain reliable macroscopic properties of the hard disks fluid in the convective regime, even for such a small $N$. In this section we will perform local measurements of some hydrodynamic fields by dividing the unit box into $n_{c} \times n_{c}$ (virtual) square cells of side $\Delta=1 / n_{c}$, see Sect. 2 . In particular, we will use $n_{c}=30$ so the measured $2 d$ maps will contain 900 local data points.

To obtain an overview of the fluid behavior, we start by measuring several global observables as e.g. the kinetic energy per particle $\left(e_{c}\right)$ and its variance, the hydrodynamic kinetic energy $\left(e_{u}\right)$, or the heat current $(J)$. Other magnitudes were also measured (as e.g. average potential energy, reduced pressure, etc.) but are not show here, see Ref. [40] for a more detailed analysis. In particular, we are interested in monitoring the effect of the transition to convective flow on these global observables, and whether this transition is accompanied by an abrupt or non-analytical change in their functional behavior. The kinetic energy per particle for a given disks configuration is given by

$$
e_{c}\left(\left\{\mathbf{p}_{i}(t)\right\}\right)=\frac{1}{N} \sum_{i=1}^{N} \frac{\mathbf{p}_{i}(t)^{2}}{2 m},
$$

where we recall that $\mathbf{p}_{i}(t)$ is the linear momentum vector of the $i$-th disk. We have measured its ensemble average $\left\langle e_{c}\right\rangle$ (or simply $e_{c}$ for notation simplicity) and its second central moment $m\left(e_{c}, 2\right) \equiv\left\langle e_{c}^{2}\right\rangle-\left\langle e_{c}\right\rangle^{2}$ as steady-state time averages using the ergodic theorem. Figure 13a shows that $\left\langle e_{c}\right\rangle$ grows monotonically with $T_{0}$ in a smooth nonlinear way, with an amplitude that increases with $g$. In this way, for any fixed non-zero external gradient, increasing the external field leads to a positive variation of the fluid kinetic energy, exerting a net work on the system. Interestingly, this is a purely nonequilibrium effect which contrast with equilibrium $\left(T_{0}=T_{L}=1\right)$ phenomenology, where variations of $g$ do not affect $\left\langle e_{c}\right\rangle$. Moreover for large enough gradients, $T_{0}>15$, the kinetic energy exhibits an asymptotic linear behavior $\left\langle e_{c}\right\rangle \approx \frac{1}{3} T_{0}$, with a slope mostly independent of $g$, even though the associated temperature profiles are highly nonlinear. The scaled variance of the kinetic energy per particle, $\operatorname{Nm}\left(e_{c}, 2\right)$, exhibits a similar behavior as a function of $T_{0}$, see Fig. $13 \mathrm{~b}$, with a global smooth but nonlinear functional form depending on $g$ with linear asymptotics. Note also that, in both cases, there is no trace of any abrupt and/or singular behavior in the data reflecting the putative transition from non-convective to convective flow occurring as $T_{0}$ is varied.

The relative fluctuations of the kinetic energy per particle, as measured by $m\left(e_{c}, 2\right) /\left\langle e_{c}\right\rangle^{2}$, exhibit a much more interesting behavior with $T_{0}$, see Fig. 14a. In the presence of gravity, i.e. for any $g>0$, the relative kinetic energy fluctuations display a clear minimum at a nontrivial value of $T_{0}$. This contrasts starkly with the equilibrium case $g=0$, see Fig. 14a, for which the relative fluctuations grow monotonically with $T_{0}$. In order to estimate the minimum location for each $g>0$, we fit a generic 9th-degree polynomial to the data (see 


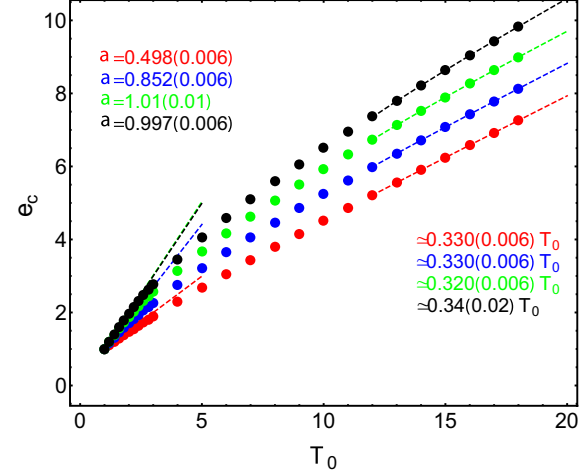

(a)

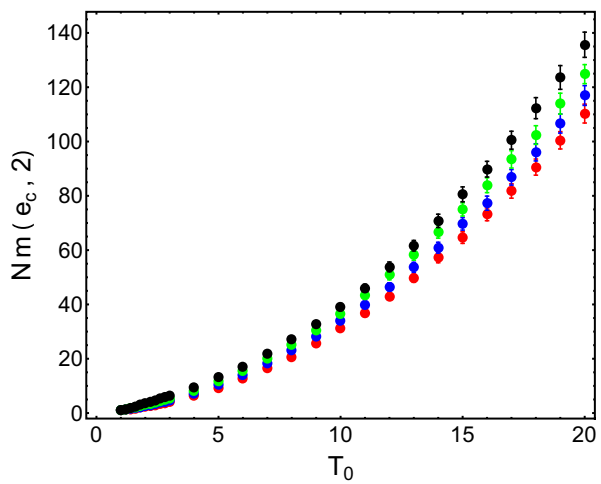

(b)

Fig. 13 a Average kinetic energy per particle, $\left\langle e_{c}\right\rangle$, as a function of $T_{0}=1,1.2, \ldots, 20$ for $g=0$ (red dots, bottom curve), $g=5$ (blue dots), $g=10$ (green dots) and $g=15$ (black dots, top curve). Note that errorbars are included in this plot and are smaller that the point size in all cases. Dashed lines show the tangent behavior of the curves at $T_{0}=1$ and the asymptotic linear behavior for large $T_{0}$ values. The legends display the measured slopes in each case. $\mathbf{b}$ Scaled variance of the kinetic energy per particle, $N m\left(e_{c}, 2\right)$, as a function of $T_{0}$. Errorbars are also included (Color figure online)

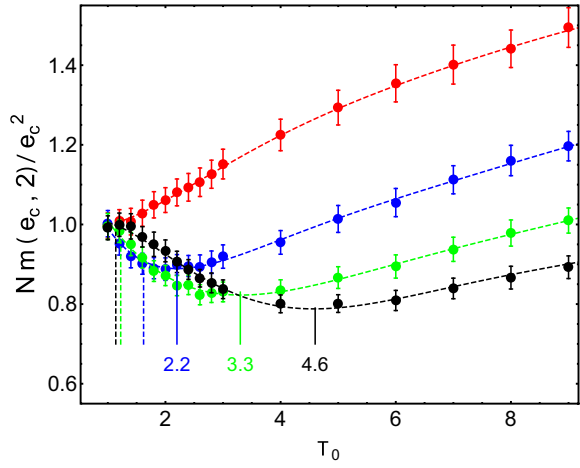

(a)

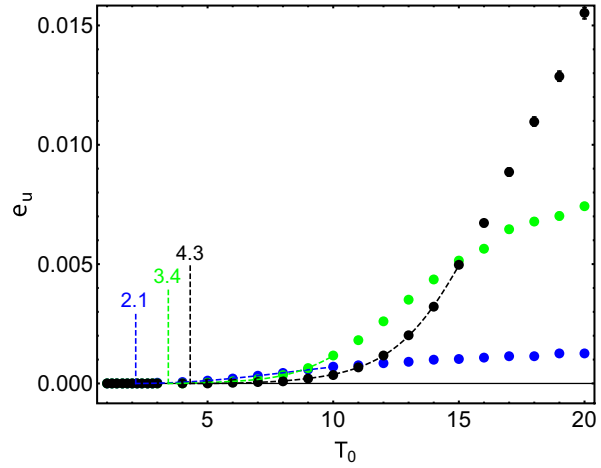

(b)

Fig. 14 a Relative variance $m\left(e_{c}, 2\right) /\left\langle e_{c}\right\rangle^{2}$ for the kinetic energy per particle. Dashed curves are phenomenological fits to the data, that allow to compute the temperature associated to the minimum relative variance. These minima are located at $T_{0}^{c}=2.2,3.3$ and 4.6 for $g=5,10$ and 15, respectively (see solid vertical lines). Dashed vertical lines correspond to the predicted values within Enskog approximation for the transport coefficients. b Average hydrodynamic kinetic energy, $\left\langle e_{u}\right\rangle$, see definition in Eq. (60), as a function of $T_{0}=1,1.2, \ldots, 20$ for $g=5$ (blue dots), $g=10$ (green dots) and $g=15$ (black dots). The curves are polynomial fits to the points (see text). Error bars are included. The dashed vertical lines locate the critical temperatures obtained from the fitted hydrodynamic kinetic energy, and the agreement with the minimum temperatures on panel a is remarkable (Color figure online)

dashed curves in Fig. 14a) and look for the temperature where its first derivative vanishes for each $g$. In this way we estimate that the minima appear at temperatures $T_{0}^{c}=2.2,3.3$ and 4.6 for $g=5,10$ and 15, respectively (solid vertical lines in Fig. 14a). One can argue that such nontrivial minima signal a threshold temperature for each $g$ separating different regimes in the fluid. In fact, we will see below that these temperatures of minimum relative kinetic energy variance correspond to the critical temperatures defining the transition to convective flow 
for each $g$. Note however that these threshold temperatures are markedly different from the critical temperature predictions based on Enskog theory (dashed vertical lines in Fig. 14a). The reason for this discrepancy lies in the Boussinesq approximation used to derive the Rayleigh critical temperatures within Enskog theory, which assumes the underlying fluid to be incompressible. Our numerical analysis shows instead that the hard disks fluid in this parameter regime behaves as a compressible fluid (as demonstrated e.g. by the non-trivial density profiles), explaining the discrepancy.

We next focus on the hydrodynamic kinetic energy, another global observable. It is defined as the kinetic energy associated to the coarse-grained velocity field. We hence need to define first the average velocity at a given spatial cell $(n, l)$, with $n, l \in\left[1, n_{c}\right]$ (see Sect. 2)

$$
\mathbf{u}(n, l ; M)=\frac{1}{N(n, l) M} \sum_{k=1}^{M} \sum_{i: r\left(i, t_{k}\right) \in B(n, l)} \mathbf{v}_{i}\left(t_{k}\right), \quad \text { with } N(n, l)=\frac{1}{M} \sum_{k=1}^{M} \sum_{i: r\left(i, t_{k}\right) \in B(n, l)} 1,
$$

with $M$ the total number of steady-state measurements at equispaced times $t_{k}, k \in[1, M]$, at the spatial domain $B(n, l)$ associated to cell $(n, l)$. We also recall that $N(n, l ; t)$ is just the number of particles at cell $(n, l)$ at time $t$, see the definition of local averages introduced in Eq. (4) of Sect. 2. The hydrodynamic kinetic energy is now defined as

$$
e_{u}(M)=\frac{1}{2 N_{C}} \sum_{(n, l)} \frac{\rho(n, l)}{\rho} \mathbf{u}(n, l ; M)^{2},
$$

where $\rho(n, l)$ is the mean packing fraction at cell $(n, l), N_{C}=n_{c}^{2}$ is the total number of (virtual) cells in which we divide the system box $\left(N_{C}=900\right.$ in our case), and the $M$ dependence in the argument indicates that the hydrodynamic kinetic energy is computed after a large but finite number of measurements $M$. In general, we expect $\mathbf{u}(n, l ; M) \approx 0$ $\forall(n, l)$ for non-convective states in the fluid $\left(T_{0}<T_{0}^{c}\right)$, while clearly one should expect $\mathbf{u}(n, l ; M) \neq 0$ once convection kicks in $\left(T_{0}>T_{0}^{c}\right)$, due to the development of spatially structured velocity fields (rolls) associated to the Rayleigh-Bénard instability. In this way the hydrodynamic kinetic energy $e_{u}(M)$ will work as an order parameter for the convection transition. Figure 14b shows the measured $e_{u}$ as a function of the hot boundary temperature $T_{0}$ for different values of $g>0$. A first observation is that the measured numerical values for $e_{u}$ are about three orders of magnitude smaller than the total kinetic energy $e_{c}$, see Fig. 13a. This is due to the hydrodynamic separation of scales, which makes the numerical analysis of the convective structures very hard. Indeed, the error analysis is particularly delicate for such a noisy data, and care is needed to extract the relevant behavior. ${ }^{5}$ As shown in Fig. 14b, the behavior of $e_{u}\left(T_{0}\right)$ is compatible with an order parameter, as argued above. In particular, we observe that the average hydrodynamic kinetic energy is very close to zero for low values of $T_{0}$, and at some nontrivial temperature which depends on $g$ it starts growing in a continuous manner. To estimate this critical temperature, we fit a piecewise-defined polynomial to the data

$$
e_{u}^{\mathrm{fit}}\left(T_{0}\right)= \begin{cases}0 & \text { for } T<T_{0}^{c}, \\ \left(T-T_{0}^{c}\right)^{2}\left(a_{0}+a_{1} T_{0}^{2}+\cdots a_{7} T_{0}^{8}\right) & \text { for } T \geq T_{0}^{c},\end{cases}
$$

in the range $T_{0} \in[2.6,12], T_{0} \in[4,13]$ and $T_{0} \in[5,16]$ for $g=5,10$ and 15, respectively, where the fitting parameters are $T_{0}^{c}$ and the $a_{i}$-coefficients, see dashed curves in Fig. 14b. We choose a leading $\left(T-T_{0}^{c}\right)^{2}$ scaling as a minimal assumption in order to demand both continuity and zero first-derivative at the transition point $T_{0}^{c}$. The values of the critical temperatures

5 We refer the reader to Ref. [40] for a detailed analysis of error propagation and correction in this case. 


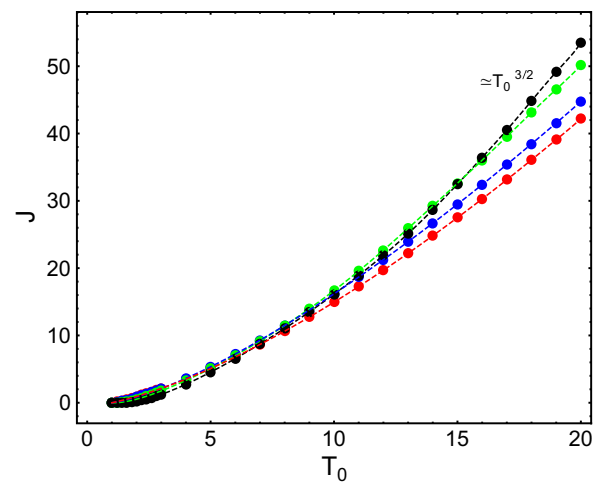

(a)

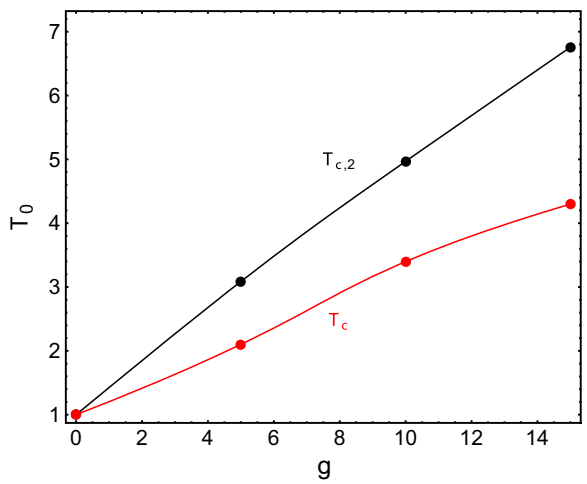

(b)

Fig. 15 a Average reduced heat current at the bottom thermal bath, $J \ell m^{1 / 2}$, as a function of $T_{0}$ for $g=0$ (red dots), 5 (blue dots), 10 (green dots) and 15 (black dots). The current measured in the opposite wall has the same magnitude and different sign, see main text. The dashed curves are fits to the data. Note that $\forall g$ the heat current grows asymptotically as $J \propto T_{0}^{3 / 2}$ for large enough $T_{0}$. b The two different critical temperatures obtained from our simulations, as a function of the gravity strength $g$. Black dots correspond to the values of $T_{0}$ at which the heat current of a system with fixed $g$ equals the one with $g=0$. Red dots are the critical temperatures obtained by fitting the hydrodynamic kinetic energy (the order parameter for the transition to convective flow) in Fig. 14b. Similar critical temperatures are obtained from the minima of the relative kinetic energy variance, see Fig. 14a (Color figure online)

so obtained are $T_{0}^{c}=2.1,3.4$ and $T_{0}^{c}=4.3$ for $g=5,10$ and 15 , respectively, see dashed vertical lines in Fig. 14b. These critical temperatures, which change only slightly when varying the polynomial degree or the fitting ranges, are fully consistent with the temperatures of minimum relative fluctuations for the kinetic energy, see Fig. 14a. We therefore conclude that these critical temperatures signal the onset of convection for the different values of $g$ in the hard disks fluid.

We briefly mention that we also measured the reduced pressure $Q(y)=P(y) \pi \ell^{2}$ at the top and bottom thermal walls (not shown [40]) in terms of the momentum flux during hard disks collisions. Interestingly, our results show that the equilibrium barometric formula $Q(0)-Q(1)=g N \pi \ell^{2}$ holds with precission in all cases, even though the fluid is out of equilibrium due to the temperature gradient and despite the non-trivial dependence of the pressure profile with $T_{0}$. Moreover, we observe that this property is independent of the state of the fluid, either convective or non-convective.

In order to characterize the transport properties of the fluid, we also measured the average heat current $J$ flowing through the fluid. In particular, we compute the energy current traversing each of the boundary thermal baths in terms of the momentum exchange during hard disks collisions. A first observation is that $J(0)=-J(1)$ so no energy accumulation happens in the system. This is of course expected due to the lack of any energy dissipation mechanism in the bulk of the fluid. Figure 15a shows the measured $J$ as a function of the hot wall temperature $T_{0}$ for $g=0,5,10$ and 15 . In all cases the heat current exhibits a smooth and nonlinear dependence on $T_{0}$, implying that the fluid's thermal conductivity is not constant and depends on the local temperature field, provided that Fourier's law applies (a conjecture that we have empirically demonstrated in the absence of gravity, see Sect. 5). Moreover, for large enough values of $T_{0}$, the heat current scales as $J \sim T_{0}^{3 / 2}$ independently of $g$, though the amplitude does depend on gravity. On the other hand, for intermediate values of $T_{0}$ a remark- 
able phenomenon appears which helps to understand the complex role of gravity in transport. In particular, note that all curves $J\left(T_{0} ; g\right)$ with fixed $g>0$ intersect the curve $J\left(T_{0} ; g=0\right)$ at a non-trivial temperature $T_{c, 2}(g)$, see Fig. 15a. This temperature separates two different regimes for any fixed $g>0$ : (i) a regime $1<T_{0}<T_{c, 2}(g)$ where gravity hinders heat transport (i.e. the heat current is smaller when gravity is present, $J\left(T_{0} ; g \neq 0\right)<J\left(T_{0} ; g=0\right)$ ), and (ii) another regime $T_{0}>T_{c, 2}(g)$ where instead gravity promotes heat transport, i.e. $J\left(T_{0} ; g \neq 0\right)>J\left(T_{0} ; g=0\right)$. We call the first regime gravity-suppressed transport regime, while the latter is termed gravity-enhanced transport regime. Figure $15 \mathrm{~b}$ shows the measured values for $T_{c, 2}$ as a function of $g$, as well as the critical temperatures $T_{c}(g)$ obtained from the convection order parameter (the hydrodynamic kinetic energy displayed on Fig. 14b). The two sets of critical temperatures are clearly different, with $T_{c, 2}(g)>T_{c}(g) \forall g>0$. This shows that convection not always enhances heat transfer. In particular, for each $g$ there is a temperature range $T_{c}(g)<T_{0}<T_{c, 2}(g)$ within the gravity-suppressed transport regime where convection has already kicked in (as reflected by a structured velocity field) but still is not efficient enough to improve the transfer of energy with respect to the gravity-free $(g=0)$ case. We call this region $T_{c}(g)<T_{0}<T_{c, 2}(g)$ semi-convective regime, to distinguish it from the fully-convective regime appearing for $T_{0}>T_{c, 2}(g)$.

To better understand the physical differences between the semi- and fully-convective regimes, we now analyze the spatial structure of the average hydrodynamic velocity field. Figure 16 shows this vector field, see definition in Eq. (59), as measured for five different hot bath temperatures $T_{0}$ and three values of $g>0$. Note that the magnitude of local hydrodynamic velocity vector is typically very small compared with the average mean particle velocity (e.g. 0.11 vs 3 for $g=10$ and $T_{0}=20$ ), a result of the hydrodynamic separation of scales which difficults the numerical analysis of convective structures. The values of $T_{0}$ shown in Fig. 16 for each $g$ have been chosen so that the first one is below $T_{c}(g)$, the second is between $T_{c}(g)$ and $T_{c, 2}(g)$, and the rest are beyond $T_{c, 2}(g)$. While disorder dominates configurations below $T_{c}(g)$ (first row in Fig. 16), for temperatures between $T_{c}(g)$ and $T_{c, 2}(g)$ some incipient local order seems to emerge though fluctuations still dominate, paving the way to fully-developed convective rolls for large enough $T_{0}$. Note that rolls for $g=5$ (bottom-left panel in Fig. 16) are noisier than the ones observed for $g=10$ or $g=15$, in particular near the hot thermal bath. The onset of convection can be better understood by looking at the streamline distribution associated with the measured hydrodynamic velocity fields. For a given velocity field, we define a streamline starting at some initial point as the trajectory whose tangent at any point corresponds to the given fixed vector field. That is, let $\mathbf{u}(x, y)=\left(u_{1}(x, y), u_{2}(x, y)\right)$ be a fixed vector field and $\left(x_{0}, y_{0}\right)$ an arbitrary initial point. Then, the streamline associated to this point is the solution of the following differential equation

$$
\frac{d y}{d x}=\frac{u_{2}(x, y)}{u_{1}(x, y)}
$$

or in parametric form

$$
\frac{d x}{d s}=u_{1}(x(s), y(s)), \quad \frac{d y}{d s}=u_{2}(x(s), y(s)),
$$

with initial condition at $\left(x_{0}, y_{0}\right)$. Numerical solutions to this problem can be simply found using e.g. a standard Runge-Kutta integrator [110]. Note that in our case the velocity vector field is defined over a discrete $(30 \times 30)$ grid, so in order to reconstruct the vector field at arbitrary points as needed by the streamline numerical integrator we perform linear interpolations from neighboring grid sites. Streamlines end whenever the underlying velocity vector field breaks the continuity of a trajectory. This can be detected by a simple stop condition in 


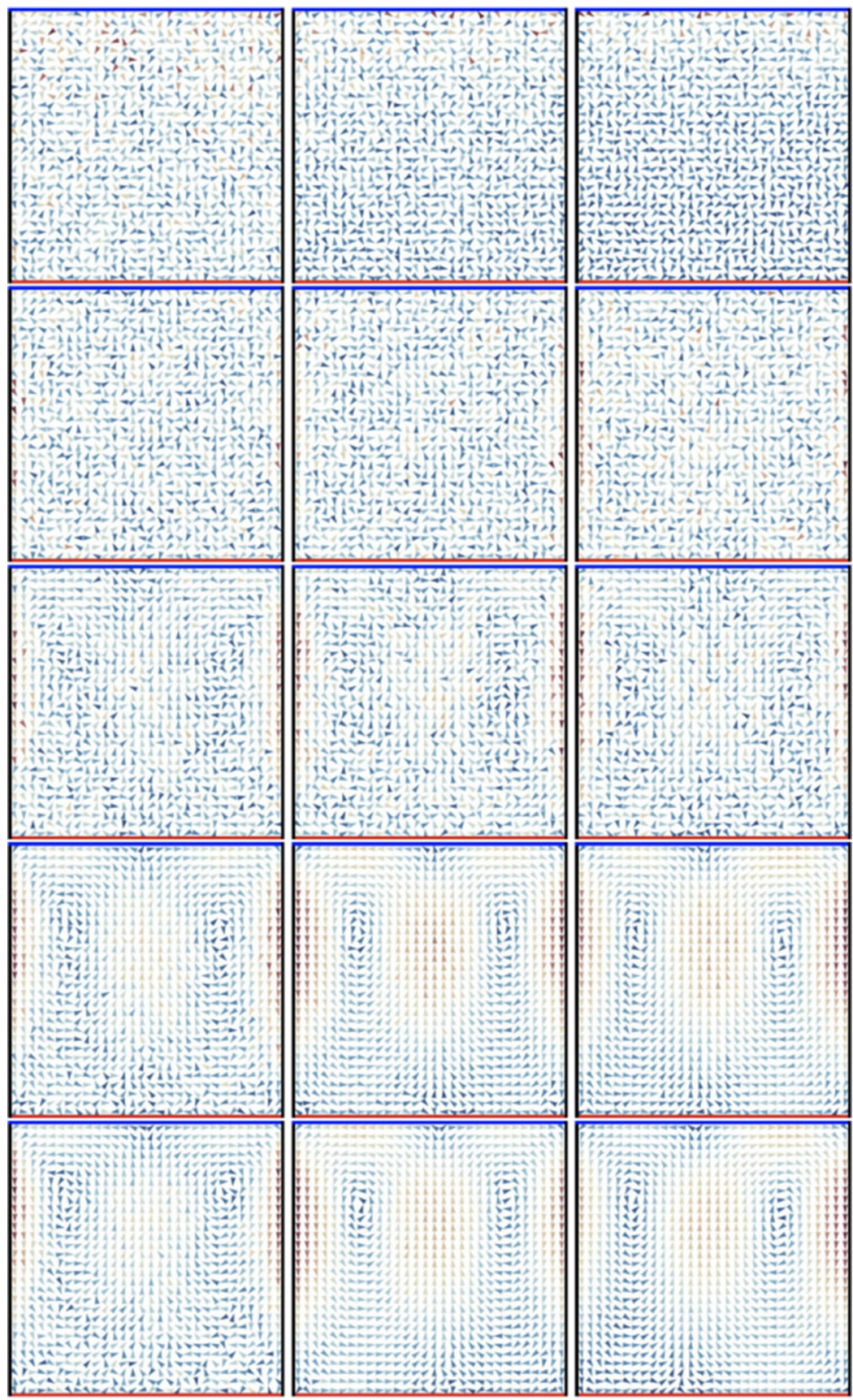

Fig. 16 Average hydrodynamic velocity field for hard disks measured across the Rayleigh-Bénard instability. Left column: $g=5$ and $T_{0}=1.4, T_{0}=2.6, T_{0}=4, T_{0}=10$ and $T_{0}=20$, from top to bottom. Central column: $g=10$ and $T_{0}=1.8, T_{0}=4, T_{0}=6, T_{0}=15$ and $T_{0}=20$, from top to bottom. Right column: $g=15$ and $T_{0}=2, T_{0}=6, T_{0}=8, T_{0}=15$ and $T_{0}=20$, from top to bottom. Note the typical roll structured associated to the Rayleigh-Bénard instability, most apparent for the largest values of $T_{0}$ 

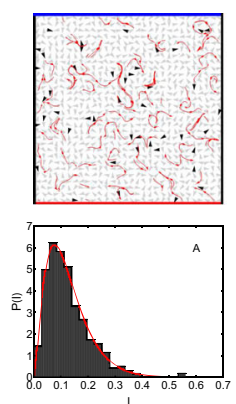
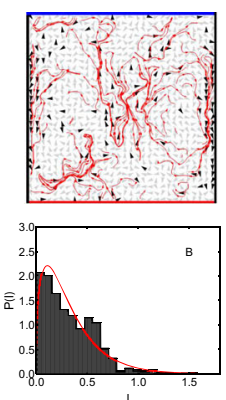
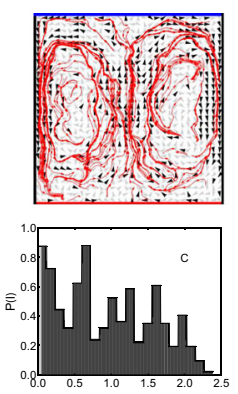
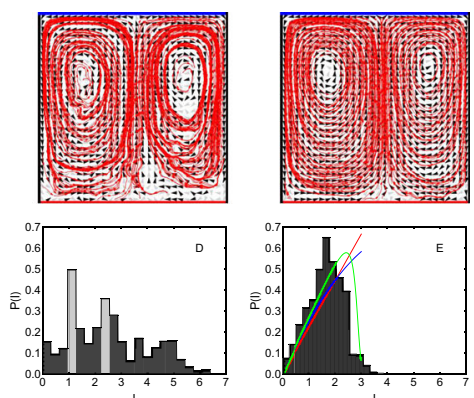

Fig. 17 Top row: Samples of $10^{2}$ streamlines (red lines) obtained from the average hydrodynamic velocity field (displayed as greyscale arrows) for $g=10$ and, from left to right, $T_{0}=1.8,4,6,10$ and 20. Streamlines grow considerably in length and increasingly follow the underlying roll structure as we cross the RayleighBénard instability. Bottom row: The corresponding probability distribution of streamline lengths measured in each case for $10^{4}$ random initial points. Solid lines are fits to the data (see text). Note the change of scale in the abcisa from left to right (Color figure online)

the above integration scheme: a streamline ends whenever the condition $\mathbf{u}(n+1) \cdot \mathbf{u}(n)<0$ is satisfied, where $\mathbf{u}(n)=\left[u_{1}\left(x\left(s_{n}\right), y\left(s_{n}\right)\right), u_{2}\left(x\left(s_{n}\right), y\left(s_{n}\right)\right)\right]$ is the velocity vector in the $n$-th step of the integration scheme. ${ }^{6}$ For any given average hydrodynamic velocity vector field we hence generate $10^{4}$ streamlines with random initial points uniformly distributed in the simulation box, and we compute the statistics of streamline path lengths. Note that the streamline length is a direct measure of the range of coherent motion observed in a fluid with a given average velocity field. The top row in Fig. 17 shows a small sample of $10^{2}$ streamlines obtained for $g=10$ and different temperatures selected to show the typical behavior at each relevant region.

The streamline length distribution changes appreciably its nature as we move from the non-convective to the fully-convective regime, see bottom panels in Fig. 17. As expected, the length distribution in the non-convective regime $T_{0}<T_{c}(g=10)=3.4$ is strongly peaked at small length values $(\sim 0.1)$, see Fig. 17a. Interestingly, the measured distributions in this regime are well fitted by a gamma distribution for all values of $g$, namely

$$
P(l ; \alpha, \beta)=\frac{l^{\alpha-1}}{\beta^{\alpha} \Gamma(\alpha)} e^{-l / \beta},
$$

with $\alpha$ and $\beta$ fitting parameters. The fitted curve for $g=10$ is indeed shown as a red line in Fig. $17 \mathrm{a}$, and the agreement with data is excellent. As we increase the hot bath temperature beyond the first critical temperature $T_{c}(g)$, the streamline length distribution widens but mantains its gamma-shaped form, see Fig. 17b. However, as we move to $T_{0}>T_{c, 2}(g=$ $10)=5$ the length distribution changes radically to an extended structure with some peaks which spreads over a broad lengths interval, see see Fig. 17c-e, losing the gamma-shaped form. This change coincides with the development of clear roll patterns in the streamlines structure. Moreover, a collection of well-defined localized channels emerge associated to the roll pattern which concentrate most long streamlines. For the largest boundary temperature, $T_{0}=20$, the distribution peaks at large values of the streamline length $(\sim 2)$, and exhibits a

6 Similar integration techniques to obtain streamlines from discrete vector fields are routinely used in imaging processing, see for instance Refs. [111,112] for some other technicalities. 

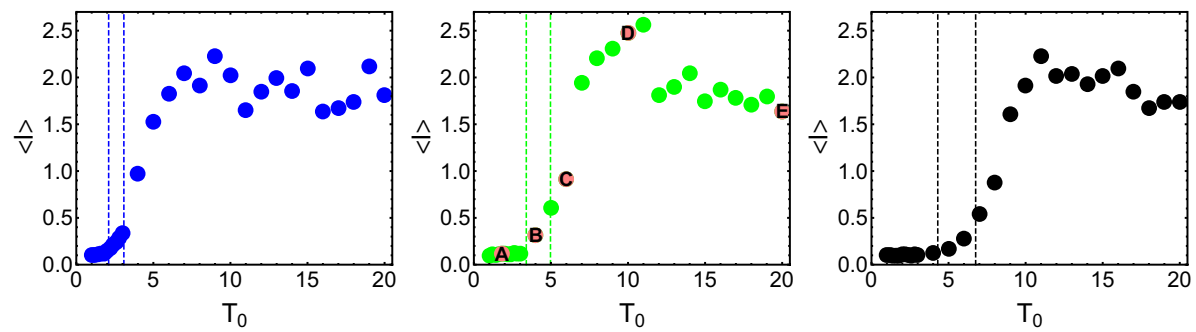

Fig. 18 Average streamline length $\langle l\rangle$ as a function of $T_{0}$ for $g=5$ (left panel), $g=10$ (central panel), and $g=15$ (right panel). In all cases $\langle l\rangle$ increases from 0 at low values of $T_{0}$ and saturates at $\langle l\rangle \approx 2$ for large enough $T_{0}$. The labels in the central $(g=10)$ panel correspond to temperatures $T_{0}=1.8(\mathbf{a}), T_{0}=4(\mathbf{b})$, $T_{0}=6(\mathbf{c}), T_{0}=10(\mathbf{d})$ and $T_{0}=20(\mathbf{e})$, i.e. the same temperatures of the streamlines snapshops and length distributions displayed in Fig. 17. The vertical dashed lines in each panel correspond to $T_{c}(g)$ and $T_{c, 2}(g)$ in each case, see Fig. 15b

sharp cutoff around $l \sim 3$, see Fig. 17e, which corresponds to closed streamlines of (maximal) length ${ }^{7}$ approximately equal to the perimeter of a rectangle of sides $1 \times 1 / 2$.

The average streamline length $\langle l\rangle$ is a good proxy for the change of behavior observed above. Figure 18 shows $\langle l\rangle$ as a function of $T_{0}$ for different values of $g>0$, together with the first and second critical temperatures in each case, $T_{c}(g)$ and $T_{c, 2}(g)$, respectively. The first critical temperature $T_{c}(g)$, associated with the onset of structure in the hydrodynamic velocity field (see Fig. 14b and related discussion), also signals the temperature where the streamline average length starts growing. For large enough values of $T_{0} \gtrsim 10,\langle l\rangle$ saturates at a constant value of around 2 corresponding to the large rolls observed in Fig. 17d, e. On the other hand, the average streamline length takes a nontrivial, nonzero value at $T_{0}=T_{c, 2}(\mathrm{~g})$, which signals the temperature where convective heat transport becomes efficient.

To better understand the physical origin of the transition to efficient convective transport, we recall that the average streamline length $\langle l\rangle$ is a measure of the range of coherent motion in the fluid. Now, the fraction $p_{r}$ of spatial cells where coherent motion happens can be identified with the fraction of hydrodynamic velocity vectors whose modulus is larger than its standard deviation, i.e.

$$
p_{r}=\frac{N_{C}^{(a)}}{N_{C}},
$$

with $N_{C}^{(a)} \leq N_{C}$ the number of active spatial cells. This means that motion in the given cell is significant against the naturally-ocurring fluctuations. The fraction $p_{r}$ of active cells where coherent motion happens grows with $T_{0}$, but also the typical lengthscale of coherent motion as given by $\langle l\rangle$. In this way we can visualize one of this active patches as a disk or circular region of coherent motion of typical size $\langle l\rangle$. Figure 19 plots $p_{r}\left(T_{0}\right)$ as a function of $\langle l\rangle\left(T_{0}\right)$ for all measured values of $T_{0}$ and $g$, and a striking collapse of curves is observed, specially in the range of moderate values of $\langle l\rangle$ where the transition to efficient convective transport happens. Indeed, one can check that, remarkably, $\langle l\rangle\left(T_{0}=T_{c, 2}(g)\right) \approx 0.6 \forall g>0$, or equivalently $p_{r}\left(T_{0}=T_{c, 2}(g)\right) \approx 0.68$ according to the scaling observed in Fig. 19. Such critical fraction of active cells (where coherent motion happens) corresponds very

\footnotetext{
${ }^{7}$ Note that Fig. $17 \mathrm{~d}$ is compatible with the existence of streamlines with length $3<l<6$, in apparent contradiction with the maximal length reported here. These very long streamlines correspond to spiraling trajectories around rolls, which do not close on themselves. Such spurious structures (that appear due to the discreteness of the underlying velocity vector field and do not affect our discussion here) can be substracted and disappear for larger $T_{0}$ [40].
} 


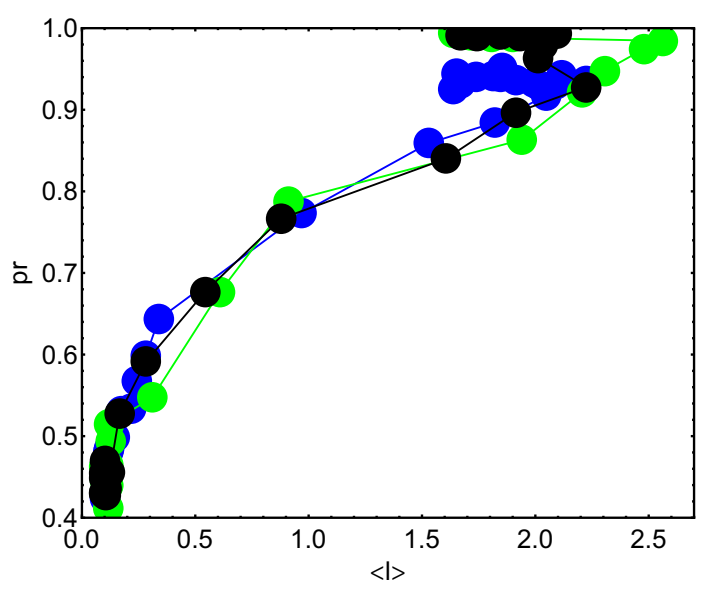

Fig. 19 Fraction $p_{r}\left(T_{0}\right)$ of spatial cells where coherent motion happens (defined as the ratio of vectors whose modulus are larger than its standard deviation) as a function of the average streamline length $\langle l\rangle\left(T_{0}\right)$ for $g=5$ (blue dots), $g=10$ (green dots), and $g=15$ (black dots). Notice the striking collapse observed for all curves, specially in the range of $\langle l\rangle$ where the transition to efficient convective transport happens. In particular $\langle l\rangle\left(T_{0}=T_{c, 2}(g)\right) \approx 0.6$ in all cases, which corresponds to $p_{r}\left(T_{0}=T_{c, 2}(g)\right) \approx 0.68$, very close to the critical covered area fraction $\phi_{c} \approx 0.676$ for two-dimensional continuous percolation of overlapping randomly-placed disks of radius $\langle l\rangle$ [113] (Color figure online)

closely to the critical covered area fraction for two-dimensional continuous percolation of overlapping randomly-placed disks of radius $\langle l\rangle$ (namely $\phi_{c} \approx 0.676$ [113]). This strongly suggests that efficient convective heat transport (i.e. heat currents above the $g=0$ limit) only happens when a percolating, macroscopically connected cluster of regions of coherent transport appears connecting the hot reservoir at the bottom with the cold bath at the top. These results hence support a two-steps picture for the transition to convective flow. As the hot bath temperature is increased, the fluid reaches a first critical temperature $T_{c}(g)$ where the hydrodynamic velocity field develops incipient structure and coherent local motions kick in. Such coherent, convection-like motions are however local and disconnected, leading to unefficient heat transport. As the hot boundary temperature keeps increasing, the density and size of such active, coherent regions increase, leading eventually to a continuous percolation transition at a second critical temperature $T_{c, 2}(g)>T_{c}(g)$ where a spanning cluster of active patches emerges connecting the hot and cold reservoirs and thus leading to efficient heat transport. A natural question concerns the dependence of the semi-convective regime $T_{0} \in\left[T_{c}(g), T_{c, 2}(g)\right]$ on the system size, a problem that we cannot address at this point. This would imply the existence of intermediate solutions to the Navier-Stokes equations, different from the well-known regular convective ones. The chances are that the two-step picture for the convection transition, that can be tested in laboratory experiments, survives in the thermodynamic limit, i.e. as $N \rightarrow \infty$.

To end this section, and in the spirit of Sect. 6, we want to test whether hydrodynamic profiles in convective flow exhibit some type of scaling into an universal master curve. To do so, we focus again on the average hydrodynamic velocity field, and leave other hydrodynamic observables for an extended analysis [40]. Figure 20 shows the $x$ - and $y$-components of the average hydrodynamic velocity vector field, $\mathbf{u}(x, y)=\left(u_{1}(x, y), u_{2}(x, y)\right)$, measured for $g=10$ and the values of $T_{0}$ already reported in Fig. 17. We observe how for $T_{0}>T_{c}(g)$ a well-defined spatial structure for each velocity component emerges, a pattern compatible 


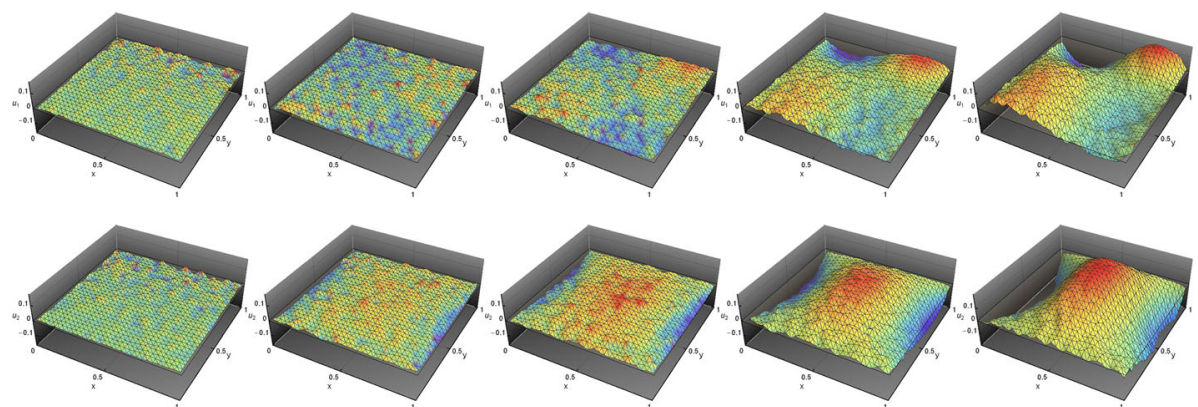

Fig. 20 Average hydrodynamic velocity field components $u_{1}$ and $u_{2}$ (top and bottom rows, respectively) measured for $g=10$ and, from left to right, $T_{0}=1.8,4,6,10$ and 20 . A velocity vector field structure compatible with convective rolls is clearly observed for large enough values of $T_{0}$, see also Fig. 16

with the convective rolls observed in Figs. 16 and 17 that gets sharper as $T_{0}$ increases. In order to search for common scaling forms, we substract from each component of the velocity field its spatial average $\bar{u}_{1,2}$, and scale the resulting field by its local standard deviation $\sigma_{1,2}$, with

$$
\sigma_{1,2}=\left[\frac{1}{N_{C}} \sum_{(x, y)}\left(u_{1,2}(x, y)-\bar{u}_{1,2}\right)^{2}\right]^{1 / 2}, \quad \bar{u}_{1,2}=\frac{1}{N_{C}} \sum_{(x, y)} u_{1,2}(x, y),
$$

where $N_{C}$ is the number of discrete spatial cells and the sum runs over all $(x, y)$-cells. In this way, we define the components of the rescaled hydrodynamic velocity field as

$$
u_{1,2}^{(s)}(x, y)=\frac{u_{1,2}(x, y)-\bar{u}_{1,2}}{\sigma_{1,2}} .
$$

Figure 21 shows the superposition of four different scaled velocity fields for large values of $T_{0}$ and gravity field values $g=5,10$ and 15 . The collapse of the scaled profiles is excellent in all cases, and one can show numerically that there is no systematic $T_{0}$-dependence on the scaled configurations [40]. Moreover, a similar scaling analysis can be performed for other hydrodynamic fields [40]. These data collapses hence demonstrate the existence of universal master curves for hydrodynamic fields in convective flow, at least in the asymptotic large- $T_{0}$ regime.

\section{Efficiency of Boundary Dissipation}

In this section we use hard disks as a model system where to study an important and pervasive question in nonequilibrium physics: can bulk forcing be balanced by a boundary dissipation mechanism? [41] Historically, bulk dissipation has been often assumed to investigate steady states in driven, nonequilibrium systems. This is the case of Drude's theory of electric conduction [114], for instance, where three different actors interplay, namely (i) a constant external force accelerating particles along a given (periodic or infinite) direction, (ii) a thermal bath which absorbs excess energy and leads the system to a steady state, and (iii) a number of random scatterers (or impurities) to trigger chaotic particle dynamics. Under these circumstances a steady state with a net current of particles flowing in the direction of the external field is achieved, due to the balance between the energy input by the external field and the 

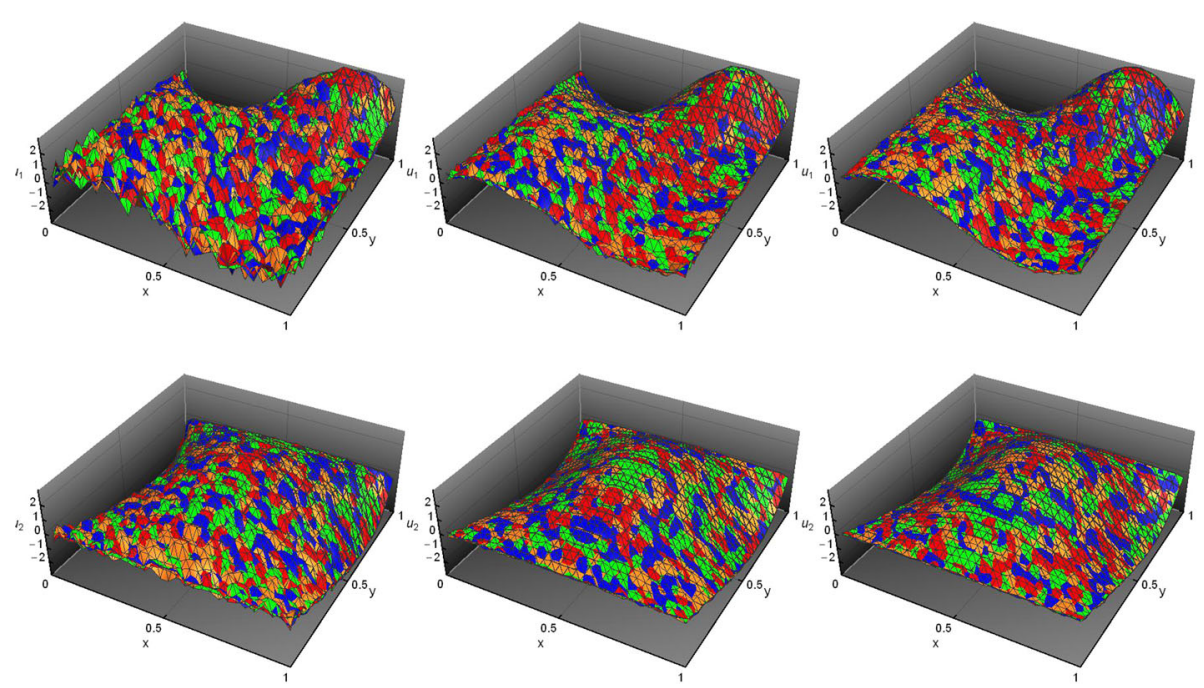

Fig. 21 Components $u_{1}^{(s)}(x, y)$ (top row) and $u_{2}^{(s)}(x, y)$ (bottom row) of the rescaled hydrodynamic velocity field for large enough values of $T_{0}$ and $g=5$ (left column), $g=10$ (central column), and $g=15$ (right column). Different colors are used for $T_{0}=17,18,19$ and 20. Note the excellent collapse found in all cases, demonstrating the existence of universal master curves in the asymptotic, large- $T_{0}$ convective regime

energy dissipated by the thermal bath. This is of course expected as both the external forcing and the thermostat are bulk mechanisms, a result that has been confirmed in simulations [114]. A natural question, motivated by realistic applications, is thus whether a boundary thermostating mechanism in the absence of bulk impurities will be capable of driving the system to a well-defined steady state. The answer is not clear as the external field tends to align particle trajectories, and dissipation in this case is a boundary effect competing with bulk driving. Simulations of Poseuille flow in a weak-flow regime [115-117] suggest that a well-defined steady state exists when boundary thermostats (balancing a bulk driving) interact strongly with the system via long-range forces. But the question remains as to whether boundary thermostats with short-range interactions can be efficient enough to balance a bulk driving. Note that efficiency of a thermostat in this context means that the reservoir is capable of absorbing enough energy to prevent an indefinite growth of energy in the driven system.

To answer this question, we perform simulations of hard disks driven by an external constant field and in contact with boundary Gaussian thermostats [41], see Fig. 22a. As before, the model consists in $N$ hard disks of radius $\ell$ confined in a two-dimensional unit box (i.e. with side $L=1$ ), and initially located on a triangular lattice structure. The simulation box is divided in three different sectors, namely a central bulk region of width $1-2 \alpha$ subject to periodic boundary conditions along the $y$-direction, and two equal lateral stripes of width $\alpha=1 / 4$ that correspond to the boundary baths. The actual numbers of disks placed in the bulk and bath regions are controlled by the corresponding number densities, $\rho_{\text {bulk }}$ and $\rho_{\text {bath }}$, respectively. For the simulations in this section we choose to work with $N=4937$ disks and densities $\rho_{\text {bulk }}=0.4$ and $\rho_{\text {bath }}=0.5$, which corresponds to $N_{\text {bulk }}=$ 2301 disks in the central sector and $N_{\text {bath }}=1318$ particles in each bath. We work at a moderate global packing fraction $\eta=N \pi \ell^{2} / L^{2} \approx 0.447$ which corresponds to disks radius $\ell \approx 5.37 \times 10^{-3}$ for $N=4937$ disks. The disks dynamics depends on the sector they are located at, see Fig. 22a. Disks in the bulk interact via elastic collisions upon contact, 


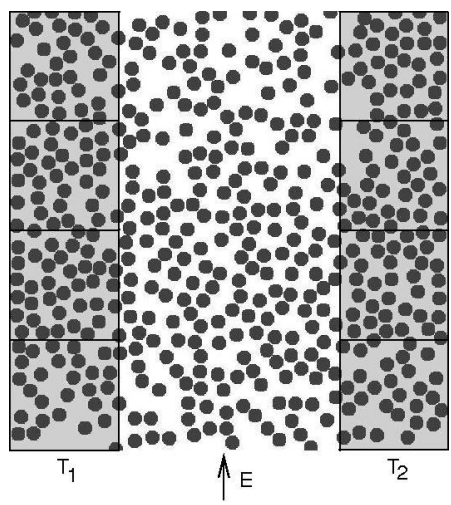

(a)

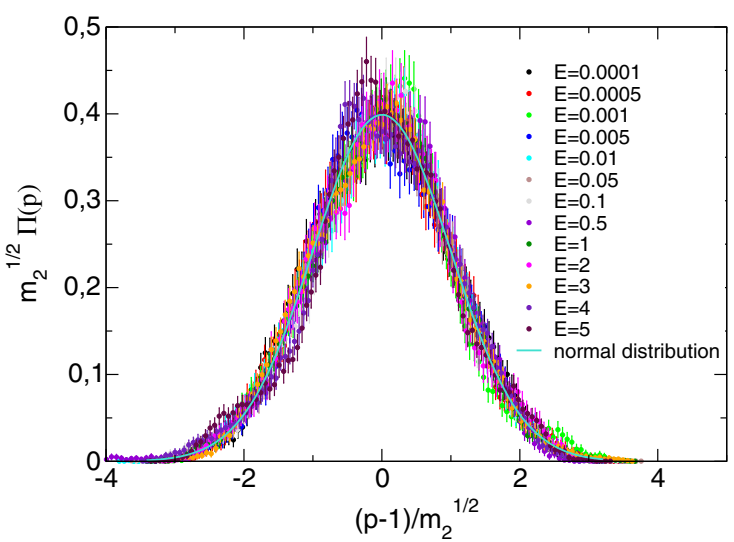

(b)

Fig. 22 a Snapshot of a typical configuration of the hard disks system in contact with Gaussian thermostats. The disks in the bulk (white area) are accelerated in the $y$-direction by a driving field $E$, which together with the periodic boundaries in this direction result in a net current of particles in the steady state. Disks at the grey boxes act as deterministic thermal baths, keeping constant their total kinetic energies for all times (as corresponds to temperatures $T_{1}$ and $T_{2}$ ). Disks collide elastically with each other, and their centers may also collide elastically with walls (represented as thick black lines). b Probability density function $\Pi(p)$ for the relative particle current $p(t)=J(t) /\langle J\rangle$, with $\langle J\rangle$ the average current, measured at the steady state for different external fields $E . m_{2}(E)$ is the measured variance of the relative current $p(t)$. The solid line is a normal distribution with zero mean and variance one

and they are also subject to a uniform acceleration of magnitude $E$ in the $y$-direction. This external field, together with the periodic boundaries along this direction in the bulk, favor the appearance of a net particle current. On the other hand, disks in the bath stripes move at constant velocity in between collisions, unaffected by the (bulk) external field. Moreover, when the center of a disk hits any of the walls that define the region in which it is contained, it is elastically reflected. In this way we keep disks confined at their respective sectors. Note that interactions between bulk and bath particles only happen via collisions across the walls. To avoid the bath disks having a net motion along the $y$-direction induced by the interaction with bulk disks, we further introduce in each bath stripe four equidistant walls along the $x$-direction, see Fig. 22a. The Gaussian thermostats work by keeping the total kinetic energy $K_{1,2}$ constant in each bath stripe, $K_{1,2}=N_{\text {bath }} T_{1,2}$, with $T_{1,2}$ the bath temperatures. This is achieved by the following prescription: when a bath disk collides with a bulk disk, the kinetic energy increment $\Delta$ (positive or negative) that the bath particle suffers is immediately shared with all other bath particles by rescaling their velocities by a factor $\left(1+\Delta / K_{1,2}\right)^{-1 / 2}$. This mechanism hence mantains constant the bath total kinetic energy. This thermostating mechanism is fundamentaly different from the stochastic thermal walls used in previous sections. The idea behind the Gaussian thermostat is to provide a heat bath mechanism capable of thermalizing a particle system while preserving the time reversibility of the particles microscopic dynamics. This has proven important e.g. to study in computer simulations the emergence of macroscopic irreversibility from time-reversible microscopic dynamics [29,50,55-58]. In order to relax the initial state and homogeinize the fluid, we let the system evolve during $100 \mathrm{~N}$ collisions in the absence of external field, $E=0$. We then switch on the external field in the bulk, measuring the observables of interest every $N$ collisions 


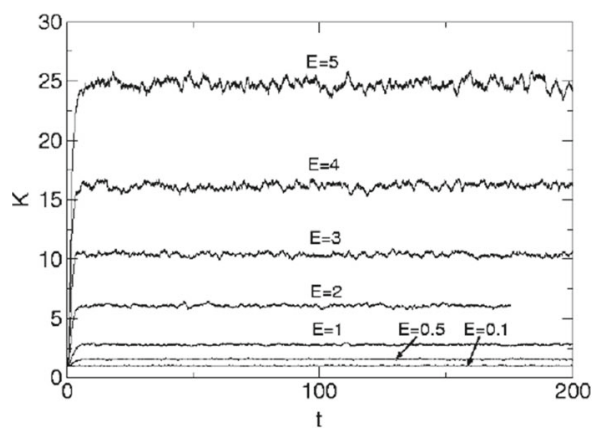

(a)

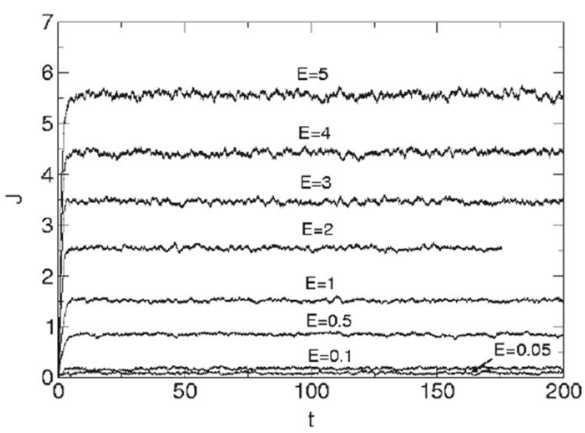

(b)

Fig. 23 Time evolution of the kinetic energy per particle $K$ (a) and the particle current $J$ (b) for different values of the driving field $E$. After a short relaxation transient, both observables reach an apparently constant value, fluctuating around it in a stable way, a behavior compatible with the existence of a well-defined steady state

during $10^{5} N$ collisions. Our simulations include data for driving fields $E \in\left[10^{-4}, 5\right]$ and equal bath temperatures $T_{1}=T_{2}=1$.

Figure 23 shows the typical time evolutions of both the kinetic energy per particle and the average particle current along the field direction, for different values of $E$. These are observables are defined as

$$
K(t)=\frac{1}{2 N} \sum_{i=1}^{N_{\text {bulk }}} \mathbf{v}_{i}(t)^{2}, \quad J(t)=\frac{1}{N} \sum_{i=1}^{N_{\text {bulk }}} v_{y, i}(t),
$$

respectively. After some short initial transient, both observables reach an apparently constant value, fluctuating around it in a stable way, a behavior compatible with the existence of a well-defined steady state of constant average kinetic energy and particle current. Figure 24a shows the measured steady-state average kinetic energy and particle current, $\langle K\rangle$ and $\langle J\rangle$ respectively, as a function of the field intensity $E$. This shows that the hard disks system response to the external electric field is in general nonlinear. In particular, the average kinetic energy per particle grows quadratically with $E,\langle K\rangle \propto E^{2}$, for large enough fields. On the other hand, the average particle current increases linearly with $E$. In particular, for small enough $E$ we observe Ohm's law of electrical conduction with a pretty large electrical conductivity, namely $\langle J\rangle \approx 1.7 E$, see also the inset to Fig. 24a. In this way, our data support a picture where the interaction of the fluid with the boundary heat baths is strong enough to effectively disorder bulk particles, despite the field tendency to align their trajectories. We also studied the internal kinetic energy $K_{I}=K-J^{2} / 2$, i.e. the kinetic energy per particle once the kinetic energy associated to the center-of-mass motion along the $y$-direction has been subtracted, finding again a quadratic dependence with the driving field $E$, see Fig. 24a.

We have also studied the statistics of fluctuations of both the current $J(t)$ and the kinetic energy $K(t)$ around its stationary average value. In particular, Fig. 22b shows the measured probability density function $\Pi(p)$ for the relative particle current, $p(t)=J(t) /\langle J\rangle$. For small values of the driving field, $E \leq 1$, the measured distribution is compatible with a Gaussian distribution with an average value 1 and a variance $m_{2}(E)$ that depends on the electric field. However, weak but systematic deviations from Gaussian statistics are observed for larger electric fields. Moreover, Fig. 24b shows the measured variance $m_{2}(E)$ for both the relative current $J(t) / J$ and the relative kinetic energy $K(t) / K$ obtained for different values 


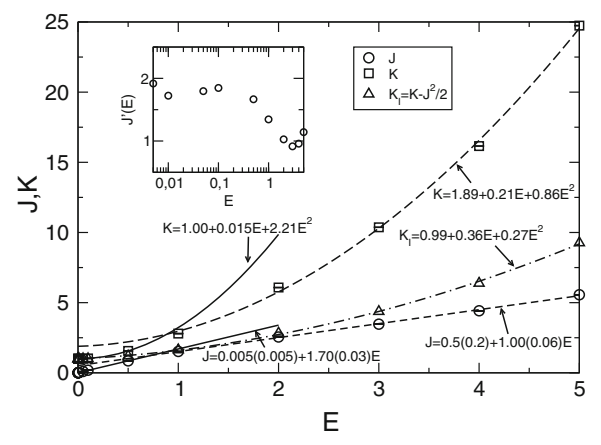

(a)

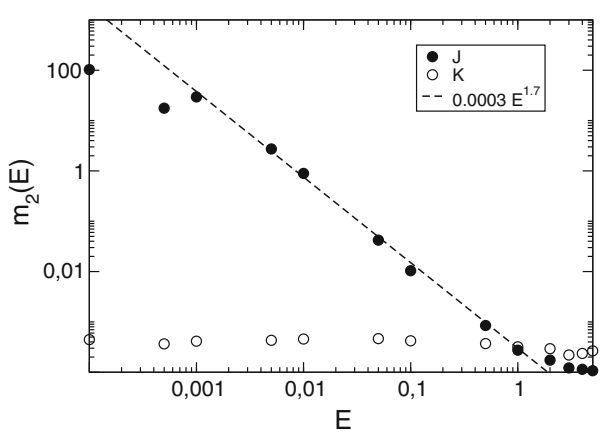

(b)

Fig. 24 a Average kinetic energy per particle $\langle K\rangle$ and average particle current $\langle J\rangle$ measured in the steady state in the bulk sector. Lines are (linear or quadratic) polynomial fits to the data, and their legends include the errors of the fitted coefficients. The internal kinetic energy per particle, $K_{I}=K-J^{2} / 2$, is also shown. Error bars are present in all cases. The inset shows the forward discrete derivative of $\langle J\rangle(E)$, which takes an approximately constant value $\approx 1.7$ for $E \ll 1$, compatible with Ohm's law of electrical conduction in this regime. b Measured variance $m_{2}(E)$ of the relative particle current $J(t) /\langle J\rangle$ and the relative kinetic energy $K(t) /\langle K\rangle$ as a function of the electric field intensity $E$. The dashed line is guide to the eye showing the power law behavior $\sim E^{-1.7}$ of the particle current variance

of $E$. Interestingly, while $m_{2}(E)$ for the kinetic energy depends only weakly on $E$, saturating at a constant value for $E \leq 1$, the fluctuations of the relative current grow as a power-law as $E \rightarrow 0$, i.e. $m_{2}(E) \sim J^{-1.7}$ in the range $E \in\left[10^{-3}, 1\right]$. This means in particular that for $E \ll 1$ a large fraction of particles are able to move against the external driving, i.e. in the $-E$ direction. This behavior is however strongly suppressed as the field increases and, for $E>1$ most of the particles move along the field.

Our results in this section hence prove how short-ranged boundary thermostats can balance a bulk driving mechanism in the absence of additional disordering effects, leading to an interesting nonequilibrium steady state characterized by stable values of different macroscopic observables, as well as a well-defined statistics.

\section{Bonus Track: Scaling Insights on Anomalous Transport in 1d}

The microscopic understanding of Fourier's law of heat conduction [7] remains as one of the most important and challenging open problems in nonequilibrium statistical physics, with no rigorous mathematical derivation to date $[8,74,87,118-120]$. Since the discovery of fat tails in the time-correlation functions of fluids [17-19,62], it has been clear that Fourier's law in low dimensions $(d<3)$ is typically anomalous, with a size-dependent heat conductivity transport coefficient $\kappa_{L}(\rho, T)$, though the nature of the anomaly remains puzzling. In two dimensions $(2 d)$ the anomaly is expected to be marginal, giving rise to a very weak, logarithmic divergence of the heat conductivity with the system size $L$ [19]. We have already offered clear numerical evidences of this weak anomaly in our simulations of heat transport for hard disks in the absence of gravity [33], see Sect. 6 and inset in Fig. 11. For onedimensional $(1 d)$ systems with momentum conservation the anomaly is expected to lead to a stronger power-law divergence, $\kappa_{L}(\rho, T) \propto L^{\alpha}$, and the conventional wisdom is that the anomaly exponent $\alpha$ is universal and belongs in the Lévy/Kardar-Parisi-Zhang universality class $[8,74,87,119,120]$, an idea supported by renormalization-group arguments [25] 
and mode-coupling approximations [121], and reinforced by recent related breakthroughs from nonlinear fluctuating hydrodynamics [122-126]. The understanding of this anomaly has attracted a lot of attention in recent years [8,17-19,25,26,33,74,87,119-148], not only because it is expected to shed light on the key ingredients behind Fourier's law at a fundamental level, but also because of its technological relevance in low-dimensional real-world materials [120,127-138].

In this section we depart from the hard disks model, the main focus of this paper, to study anomalous transport in a related $1 d$ hard-particle model using the novel scaling method already presented in Sect. 6 [39]. Previous attempts to characterize the transport anomaly in $1 d$ have been mostly based on linear response theory, and hence were critically-dependent on a large system-size limit which is in fact never attained [146]. In comparison, the scaling method used here [39] takes full advantage of the nonlinear character of the heat conduction problem in a natural way, allowing to disentangle the crucial bulk size scaling from the artificial boundary finite-size corrections [39]. Our model in this section is the archetypical $1 d$ diatomic hard-point gas in a temperature gradient [22-24,149-156], which is characterized by the mass ratio $\mu \equiv M / m>1$ between neighboring particles. It is a $1 d$ Hamiltonian model fluid consisting in $N$ hard-point particles with alternating masses, $m=1$ and $M=\mu m>1$, moving ballistically in a line of length $L$ in between elastic collisions with neighboring particles. ${ }^{8}$ In the spirit of previous sections, see e.g. Sects. $2-7$, the fluid is coupled to two stochastic thermal walls at the boundaries, $x=0, L$, which reflect particles upon collision with a velocity modulus randomly drawn from a Maxwellian distribution defined by the wall temperature $T_{0, L}[8,74,87,119]$, see Eq. (2) above. For $T_{0} \neq T_{L}$, the boundary gradient drives the system to an inhomogeneous nonequilibrium steady state characterized by nonlinear number density and temperature profiles, $\rho(x)$ and $T(x)$ respectively $[8,74,87,119,120]$. Such nonlinearity is a clear indication of the nontrivial dependence of the heat conductivity on $\rho(x)$ and $T(x)$.

In order to characterize the steady-state hydrodynamic profiles, we performed a large number of event-driven simulations of the $1 d$ diatomic gas for a broad set of boundary reservoir temperatures $T_{0}=2,5,10,20$ (with fixed $T_{L}=1$ ), global number densities $\eta \equiv N / L=0.5,1,2,3$, different mass ratios $\mu=1.3,1.618,2.2,3,5,10,30,100$, and numbers of particles $N=101,317,1001,3163,10001$, reaching up to $N=10^{5}+1$ in some cases. We measured locally different relevant magnitudes including the local kinetic energy, number density, virial pressure and energy current density, as well as the energy current flowing through the thermal baths at $x=0, L$ and the pressure exerted on these fixed walls in terms of momentum exchange. As observed for hard disks in $2 d$, see Sect. 6 , the observables measured at the walls (pressure and current) agree in all cases with their bulk counterparts, which are constant across the system. For local measurements we divided the fluid into 30 virtual cells, a constant number independent of other system parameters. Time in simulations was measured in units of $t_{0}=\sqrt{M /\left(2 T_{L} \eta^{2}\right)}$, the mean free time of a heavy particle at temperature $T_{L}$, and time averages were performed taking into account the relaxation and correlation timescales of the $1 d$ fluid, which grow strongly with $N$ (see Ref. [39] for details). Statistical errors are computed in all cases at $99.7 \%$ (i.e. $3 \sigma$ ) confidence level, and error bars are shown if larger than plotted symbols.

Figure 25 shows the temperature and density profiles measured for $\mu=3$ and varying $T_{0}$, $\eta$ and $N$. Note that similar hydrodynamic profiles are obtained for all other $\mu$ 's (not shown). These profiles are clearly nonlinear, as expected for a heat conductivity $\kappa_{L}(\rho, T)$ that depends

8 Note that the mass difference between neighbors is needed in order for the model to be ergodic. Equal-mass hard particles in $1 d$ just exchange velocities upon collision, leading to non-ergodic particle dynamics (e.g. the initial set of velocities is conserved by the dynamics). 


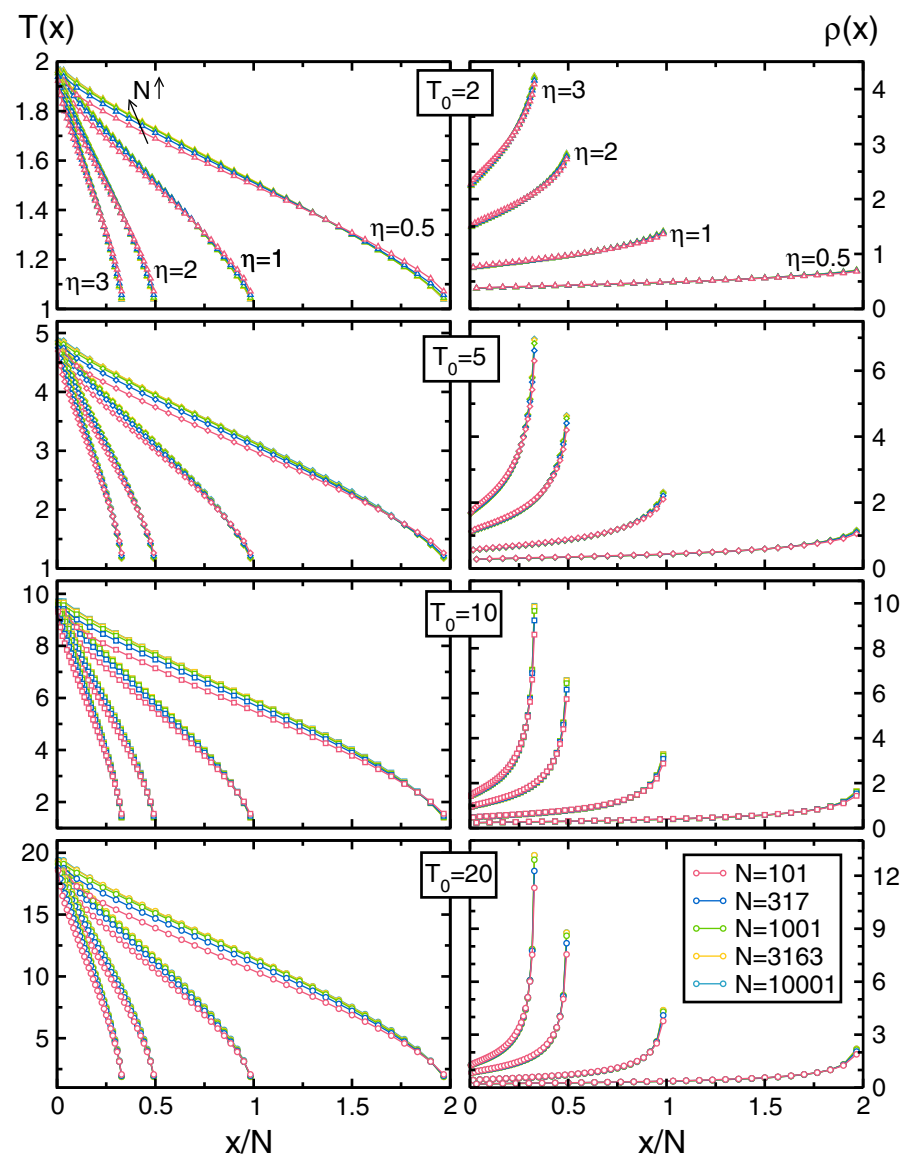

Fig. 25 Temperature (left) and density (right) profiles measured for (from top to bottom) $T_{0}=2,5,10,20$ and varying $\eta$ and $N$, for a mass ratio $\mu=3$. Similar hydrodynamic profiles are obtained for all other $\mu$ 's

on the local density and temperature, and exhibit strong finite-size effects. Our first goal is to test the macroscopic local thermodynamic equilibrium (MLTE) property directly from these data, as we already did for hard disks, see Sect. 5. MLTE conjectures that the stationary density and temperature fields are coupled locally via the equilibrium equation of state (EoS) [32], which for this model is simply the ideal gas EoS,

$$
P=\rho(x) T(x) .
$$

In order to test MLTE, we hence take the density and temperature profiles of Fig. 25 and plot in Fig. 26a the local reduced temperature, $T(x) / P$, with $P$ the finite-size pressure measured in each simulation (not shown [39]), as a function of the associated local density $\rho(x)$. Remarkably, all data comprising 2400 points from 80 different simulations for widely different systems sizes, temperature gradients and global densities, collapse onto a single curve which follows with high precision the expected ideal-gas behavior $1 / \rho$, see solid line in Fig. 26a and inset therein. Note that, interestingly, the excellent data collapse is maintained also for data points within the boundary layers near the thermal walls. Similar excellent collapses are observed for all mass ratios $\mu$ studied here. This confirms the robustness of the 


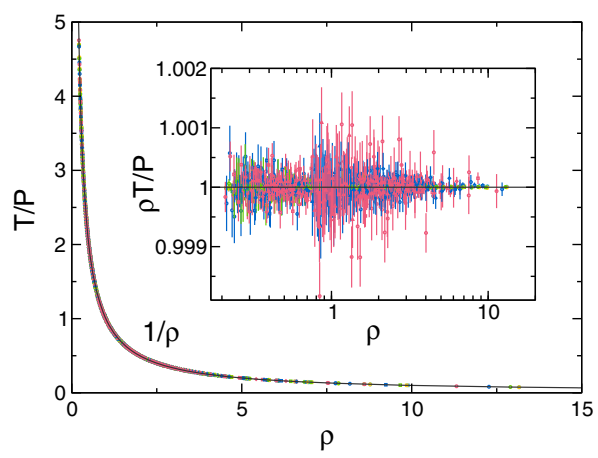

(a)

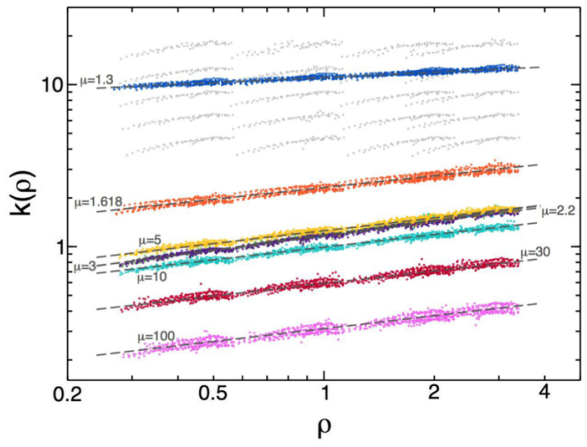

(b)

Fig. 26 a Testing macroscopic local thermodynamic equilibrium (MLTE). Measured local reduced temperature, $T(x) / P$, plotted as a function of the associated local density $\rho(x)$ for $\mu=3$ and $\forall T_{0}, \eta, N$, corresponding to all profiles displayed in Fig. 25. This plot sums up to 2400 data points from 80 different simulations. An excellent data collapse is obtained which follows with high precision the expected ideal-gas behavior $1 / \rho$, plotted as a thin line. Inset: Scaling plot of $\rho(x) T(x) / P$ vs $\rho(x)$ for the same conditions. These data show that macroscopic local equilibrium is a very robust property, even in the presence of strong finite-size corrections on the hydrodynamic profiles, see Fig. 25. b Density dependence of heat conductivity as captured by $k(\rho)$, see Eq. (69) below. Light gray points show the curves obtained for $\mu=3$ before scaling data by $L^{-\alpha}$ along the $y$-axis, while dark color curves show the scaled curves for each $\mu$. A power-law behavior is apparent in all cases. Dashed lines are power-law fits to the data, see Table 1

MLTE property far from equilibrium [32], even in the presence of both anomalous transport (see below) and important finite size effects.

We expect that, when driven out of equilibrium by a boundary temperature gradient, the $1 d$ diatomic hard-point fluid will sustain a non-vanishing heat current $J$ given by an anomalous version of Fourier's law [39]

$$
J=-\kappa_{L}(\rho, T) \frac{d T(x)}{d x}, \quad x \in[0, L],
$$

with $\kappa_{L}(\rho, T)$ a well-defined local conductivity functional which may depend on $L$. Due to the homogeneity of the interaction potential, the heat conductivity of the $1 d$ diatomic hardpoint gas exhibits a well-known density-temperature separability [33]. Moreover, standard dimensional analysis arguments show that $\kappa \propto \sqrt{T / m}$ [33], see also Sect. 3, and the known dimensional anomaly for transport implies in turn that $\kappa \propto L^{\alpha}$ at leading order, so

$$
\kappa_{L}(\rho, T)=L^{\alpha} \sqrt{T / m} k(\rho),
$$

with $k(\rho)$ a function solely dependent on density. Note that this ansatz discards possible subleading corrections in $L$. Equations (68) and (69) are not Fourier's law in the usual sense, as the latter implies a size-independent $\kappa$, while the conductivity in this case grows with the system size as $L^{\alpha}$, with $\alpha$ the anomaly exponent. Indeed, our aim now is to test the validity of Eqs. (68), (69) and measure the anomaly exponent with the simple yet powerful scaling technique already employed in Sect. $6[33,39]$. In particular, using the EoS to write $T(x)=P / \rho(x)$ and Eq. (69), we can rewrite Eq. (68) as

$$
\frac{J \sqrt{m}}{P^{3 / 2}} L^{-\alpha}=G^{\prime}(\rho) \frac{d \rho}{d x}=\frac{d G(\rho)}{d x},
$$


where we have defined a new function $G^{\prime}(\rho) \equiv k(\rho) \rho^{-5 / 2}$ (recall that ' denotes derivative with respect to the argument). This equation describes the macroscopic heat transport problem in terms of the density field $\rho(x)$, once its boundary conditions $\rho(x=0, L)=\rho_{0, L}$ have been specified. As for hard disks, see Sect. 6, these boundary conditions can be inferred from the constraints

$$
\frac{T_{0}}{T_{L}}=\frac{\rho_{L}}{\rho_{0}}, \quad \eta=\frac{1}{L} \int_{0}^{L} \rho(x) d x=\frac{\int_{\rho_{0}}^{\rho_{L}} \rho G^{\prime}(\rho) d \rho}{G\left(\rho_{L}\right)-G\left(\rho_{0}\right)} .
$$

Note that the externally controlled parameters in the problem are the temperatures of the boundary reservoirs, $T_{0}$ and $T_{L}$, and the global number density $\eta$. The pressure and the heat current can be now obtained as $P=T_{0} \rho_{0}$ and $J=P^{3 / 2}\left[G\left(\rho_{L}\right)-G\left(\rho_{0}\right)\right] /\left(L^{1-\alpha} \sqrt{m}\right)$. Now, since both $J$ and $P$ are state-dependent constants, Eq. (70) immediately implies that $G[\rho(x)]=\psi L^{-\alpha} x+\zeta$ with $\psi \equiv J \sqrt{m} / P^{3 / 2}$ the reduced current and $\zeta=G\left(\rho_{0}\right)$ a constant, i.e. $G[\rho(x)]$ is a linear function of position $x \in[0, L]$. Equivalently,

$$
\rho(x)=F\left(\frac{\psi}{L^{\alpha}} x+\zeta\right),
$$

where we assume that $G(\rho)$ has a well-defined inverse $F(z) \equiv G^{-1}(z)$, as otherwise expected from the regular behavior of the measured stationary density profiles, see e.g. Fig. 25. Therefore, according to Eq. (72), there exists a single universal master curve $F(z)$ from which any steady state density profile follows after a linear spatial scaling $x=L^{\alpha}(z-\zeta) / \psi$. This scaling behavior is automatically transferred to temperature profiles via the local EoS $P=\rho(x) T(x)$, so

$$
\frac{T(x)}{P}=\frac{1}{F\left(\frac{\psi}{L^{\alpha}} x+\zeta\right)} .
$$

These scaling laws are independent of the global density $\eta$ or the nonequilibrium driving defined by the baths temperatures $T_{0}$ and $T_{L}$, depending exclusively on the function $k(\rho)$ controlling the fluid's heat conductivity. Alternatively, Eq. (72) implies that any measured steady density profile can be collapsed onto the universal master curve $F(z)$ by scaling space by the scaled reduced current $L^{-\alpha} J \sqrt{m} / P^{3 / 2}$, with $J$ and $P$ measured in each case, and shifting the resulting profile an arbitrary constant $\zeta$ (similarly for temperature profiles). The resulting collapse is expected to be very sensitive to the anomaly exponent $\alpha$, and this suggests a simple scaling procedure to measure both $\alpha$ and the universal master curve in simulations. Furthermore, the empirical confirmation of the collapse would immediately imply that the $1 d$ diatomic hard-point gas obeys the anomalous version of Fourier's law, Eqs. (68) and (69) [39].

We hence proceed now to analyze the $1 d$ fluid's scaling behavior. Note that, as for hard disks, the boundary thermal walls act as defects (akin to fixed, infinite-mass particles) which disrupt the structure of the surrounding fluid, defining two boundary layers where finite-size corrections pile up. To avoid these spurious corrections in scaling collapses, we neglect data from these boundary layers (up to 7 cells adjacent to each wall), focusing the analysis on the remaining bulk profiles $\rho(x)$ and $T(x)$. For a given exponent value $\alpha$, each bulk density profile $\rho(x)$ is then plotted as a function of $L^{-\alpha} \psi x$, with $\psi=J \sqrt{m} / P^{3 / 2}$ measured in each case, and shifted by a constant $\zeta$ to achieve an optimal collapse among all scaled profiles, see Fig. 27a. The vector of optimal shifts $\zeta_{0}$ for fixed $\alpha$ and $\mu$ is obtained by minimizing a standard collapse metric $D(\zeta ; \alpha, \mu)$ for the density profiles $[39,157]$, which measures the 


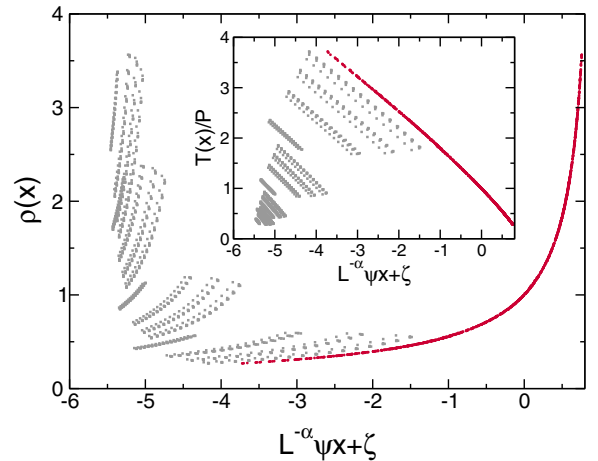

(a)

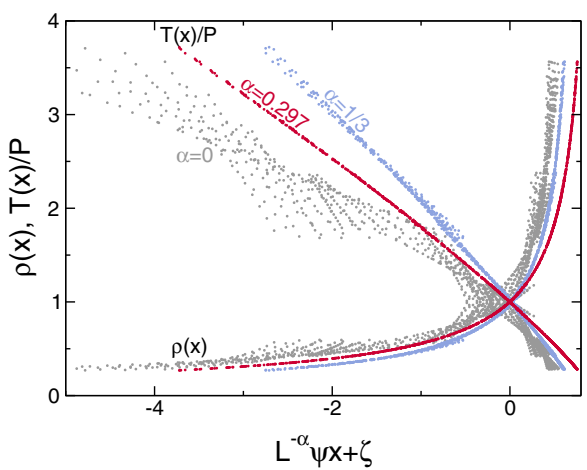

(b)

Fig. 27 Scaling procedure and data collapse. a Density profiles for $\mu=3 \forall N, T_{0}, \eta$ as a function of $L^{-\alpha} \psi x$ with $\alpha=0.297$, before (light gray) and after (dark red) the shifts $\zeta$. Inset: Same as before, but for the reduced temperature profiles. Note that the shifts are those obtained from density profiles. In both cases the data collapse is excellent. b Optimal collapse of density and reduced temperature profiles for $\mu=3$ and three different exponents $\alpha=0,0.297$, and $1 / 3$. The superior collapse for $\alpha=0.297$ is apparent. The abscisa for $\alpha=0$ has been divided by a factor 10 for the sake of clarity(Color figure online)

relative average distance among all pairs of overlapping curves, and the same shifts are used to collapse reduced temperature profiles, $T(x) / P$. The resulting data collapses are very sensitive to $\alpha$, see Fig. 27b, so the the true anomaly exponent $\alpha$ can be measured with high precision for each mass ratio $\mu$ by minimizing $D(\alpha, \mu) \equiv D\left(\zeta_{0} ; \alpha, \mu\right)$ as a function of $\alpha$. In fact, the distance function $D(\alpha, \mu)$ has a pronounced minimum in $\alpha$ for each $\mu$, see inset in Fig. 28a, whose width and depth allow to estimate the exponent error [39,157].

Figure 28a shows the measured anomaly exponent (and its error) as a function of the mass ratio $\mu$. Interestingly, the measured exponent turns out to be non-universal, depending non-monotonously on the mass ratio, $\alpha=\alpha(\mu)$, see also Table 1, growing first from small values at low $\mu$ to a maximum $\alpha \approx 0.3<1 / 3$ for $\mu=2.2$, and decaying afterwards to an asymptotic value $\alpha \approx 1 / 4$ for large $\mu$. Figure 28b shows the master curves obtained from density and reduced temperature bulk profiles for different mass ratios by using the measured $\alpha$ 's, and in all cases the resulting collapses are striking, confirming that anomalous Fourier's law (68)-(69) rules heat conduction in this $1 d$ model. This unambiguous result hence calls into question the prevailing conjecture that the anomaly in $1 d$ heat transport is universal $[25,119,121-126,139]$.

An alternative, very popular method to measure the heat conductivity of a $1 d$ fluid consists in setting the fluid with $N$ particles and density $\eta=N / L$ under a small temperature gradient, with fixed wall temperatures $T_{0, L}$, and then increasing $N$ at constant density $[74,87,119]$. For large enough $N$ the overall temperature gradient is small enough so one can approximate Fourier's law as

$$
J=-\kappa_{L}(\rho, T) \frac{d T}{d x} \approx+\tilde{\kappa} \frac{\Delta T}{L},
$$

with $\Delta T=T_{0}-T_{L}, J$ the measured current and $L=N / \eta$. In this way, the estimated heat conductivity can be obtained as $\tilde{\kappa} \approx J L / \Delta T$. This effective conductivity is expected to diverge as $N^{\gamma}$ for large enough values of $N$, though there is no way of knowing a priori which value of $N$ is large enough. What it's typically found however in actual, cutting-edge simulations is an effective anomaly exponent $\tilde{\gamma}(N)$ which exhibits itself persistent finitesize corrections [155]. This approximate method completely neglects the nonlinear density 


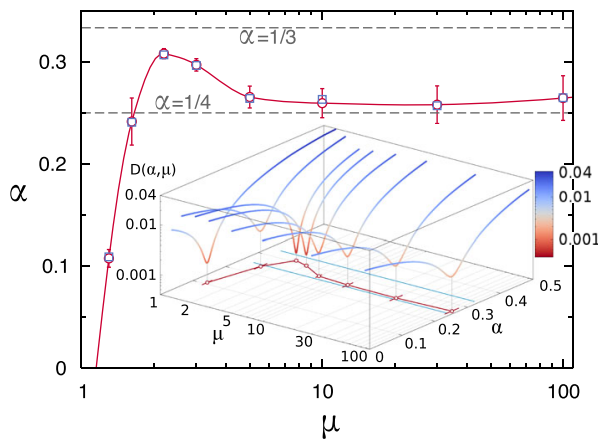

(a)

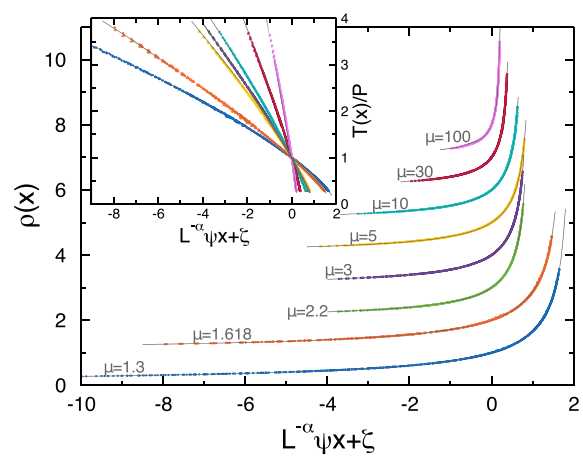

(b)

Fig. 28 Breakdown of universality and master curves in anomalous Fourier's law. a Mass ratio dependence of the anomaly exponent measured from scaling $(\bigcirc)$. The non-monotonous behavior of $\alpha(\mu)$ clearly signals the breakdown of universality for anomalous Fourier's law in $1 d$. The exponent measured from the power-law fit for $k(\rho)$ is also shown ( $\square)$, being fully compatible with $\alpha$ in each case. The line is a guide to the eye. Inset: The collapse metric $D(\alpha, \mu)$ as a function of $\alpha$ exhibits a deep and narrow minimum for each $\mu$ (note the logarithmic scale in $z$-axis), offering a precise measurement of the anomaly exponent and its error. $\mathbf{b}$ Collapse of density profiles for each $\mu$ obtained by using the measured $\alpha$ in each case. The master curves have been shifted vertically for better comparison. In all cases, the data collapse is excellent. The lines are theoretical predictions, see main text. Inset: Collapse of reduced temperature profiles for the same conditions, and theoretical curves. In all cases, each curve for fixed $\mu$ contains 1280 points measured in 80 different simulations for varying $N, T_{0}$ and $\eta$. The abscisas for the $\mu=1.3$ data have been divided by 4 to better visualize the results

Table 1 Measured anomaly exponents $\alpha$ and their error for different mass ratios $\mu$, see Fig. 28

\begin{tabular}{llll}
\hline$\mu$ & $\alpha$ & $\beta$ & $a$ \\
\hline 1.3 & $0.108(9)$ & $0.109(1)$ & $11.105(8)$ \\
1.618 & $0.242(23)$ & $0.2408(18)$ & $2.307(3)$ \\
2.2 & $0.308(5)$ & $0.3068(11)$ & $1.1765(9)$ \\
3 & $0.297(6)$ & $0.2964(11)$ & $1.1633(9)$ \\
5 & $0.266(11)$ & $0.2641(12)$ & $1.2622(12)$ \\
10 & $0.260(14)$ & $0.2632(19)$ & $0.9874(14)$ \\
30 & $0.258(18)$ & $0.257(1)$ & $0.5942(12)$ \\
100 & $0.265(22)$ & $0.2648(23)$ & $0.3095(5)$ \\
\hline
\end{tabular}

Also shown are the fitted exponent and amplitude of the power-law density dependence of the conductivity, $k(\rho)=a \rho^{\beta}$, see Fig. 26b. Notice that in all cases $\beta=\alpha$ within error bars, as predicted

and temperature dependence of the heat conductivity, and by construction it can only yield meaningful results in the limit $N \rightarrow \infty$. It is therefore no surprise that the effective anomaly exponent derived within this approach for finite $N$ varies slowly with the system size, as in fact the very definition of $\tilde{\kappa}$ above (and hence $\gamma$ ) is correct only asymptotically. A natural question is hence whether the new anomaly exponent $\alpha(\mu)$ measured with the novel scaling method described above exhibits similar finite-size corrections. A first clue that this is not the case is that, for $N \in\left[10^{2}+1,10^{4}+1\right]$, a slight change in the anomaly exponent measured with our scaling method completely destroys the observed collapse, see Fig. 27b, while the effective anomaly exponent measured with standard methods varies widely with $N$ in the same $N$-range, e.g. $\tilde{\gamma}(N) \in[0.25,0.5]$, see Fig. 3b in Ref. [155]. In any case, in order to test 


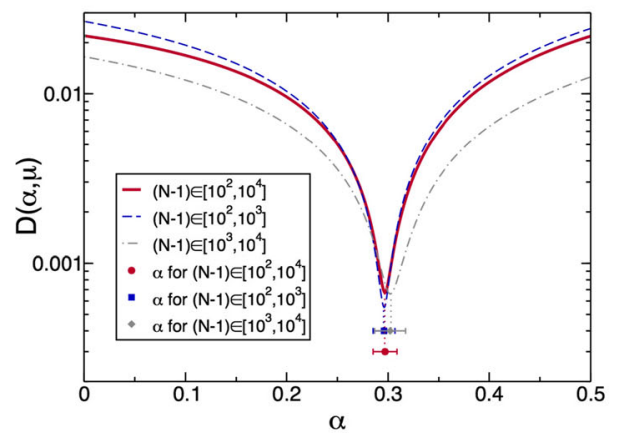

(a)

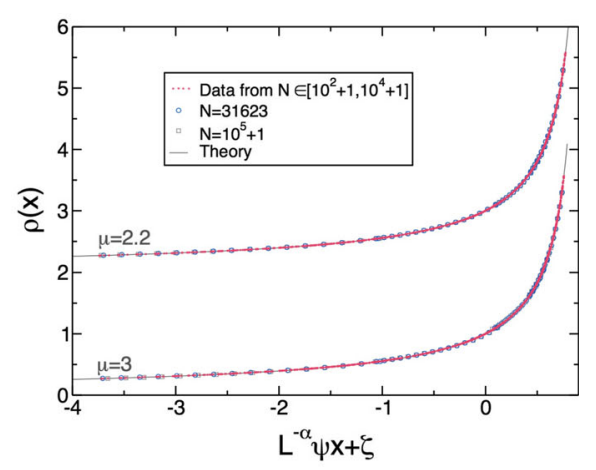

(b)

Fig. 29 a Collapse metric $D(\alpha, \mu)$ for $\mu=3$ as a function of $\alpha$ when considering all data, $N \in\left[10^{2}+1,10^{4}+\right.$ 1], $T_{0} \in[2,20], \eta \in[0.5,3]$ (full line), and when $N$ is restricted to small $N \in\left[10^{2}+1,10^{3}+1\right]$ (dashed line) or large $N \in\left[10^{3}+1,10^{4}+1\right]$ (dot-dashed line). Note the logarithmic scale in the vertical axis. The points and the errorbars below represent the estimated value of the anomaly exponent $\alpha$ in each case. The values obtained from the $N$-restricted sets are fully compatible between them and with the previous result using the combined sets, all points lying well within the errorbars. Note that the large- $N$ distance curve is slightly wider than the small- $N$ curve due to the somewhat larger uncertainties accompanying data for large $N$, as expected from the strong growth of relaxation and correlation times with $N$ [39]. b Collapse of density profiles for $\mu=2.2$ (top) and $\mu=3$ (bottom) obtained by using the measured anomaly exponent $\alpha$ in each case, see Table 1. Small points correspond to the scaling collapse obtained for $N \in\left[10^{2}+1,10^{4}+1\right], T_{0} \in[2,20]$, and $\eta \in[0.5,3]$, while bigger points correspond to additional results obtained from massive simulations for larger system sizes, namely $N=31623(\bigcirc)$ and $N=10^{5}+1(\square)$, with $T_{0}=20$ and $\eta \in[0.5,3]$. The line stands for the theoretical prediction, and the master curve for $\mu=2.2$ has been shifted vertically for better comparison

quantitatively this idea, we divided our original data into two different subsets, one for small $N \in\left[10^{2}+1,10^{3}+1\right]$ and another one for large $N \in\left[10^{3}+1,10^{4}+1\right]$. In this way both data subsets have the same amount of points, thus avoiding possible sampling issues. Next, we perform our scaling analysis on both subsets and obtain the collapse distance metric $D(\alpha, \mu)$ as a function of $\alpha$ in each case. In both cases, small $N$ vs large $N$, this function exhibits a pronounced minimum in $\alpha$ for each $\mu$, and these minima identify the anomaly exponent as measured in each subset. Figure 29a shows the results of this analysis for mass ratio $\mu=3$, and the conclusion is clearcut: the anomaly exponents measured from the small- $N$ and large$N$ subsets are fully compatible between them and with our previous measurement based on all $N \in\left[10^{2}+1,10^{4}+1\right]$, so no significant, systematic variation of the anomaly exponent with the system size is found beyond the stringent errorbars of our measurements. We found similar results for all other $\mu$ 's.

To further test for possible subtle finite-size corrections on $\alpha(\mu)$, we simulated steadystate heat transport in the diatomic hard-point fluid for $N=31623$ and $N=10^{5}+1$, i.e. one order of magnitud beyond our previous simulations. The scale of these simulations is so large that we had to restrict the region of parameter space explored. In particular, we perform simulations for these large- $N$ values, global densities $\eta=0.5,1,2,3$, a strong temperature gradient defined by $T_{0}=20$ and $T_{L}=1$, and two intermediate mass ratios $\mu=3$ and $\mu=2.2$ for which relaxation (and correlation) time scales are slightly shorter (note that for both small and large $\mu$ the fluid's relaxation and correlation times increase drastically $[158,159])$. Figure 29b shows the collapse of density profiles for $\mu=2.2$ and $\mu=3$ obtained by using the measured anomaly exponent $\alpha(\mu)$ in each case, namely $\alpha(\mu=2.2)=0.308$ 
and $\alpha(\mu=3)=0.297$, see Table 1 , once the new data for $N=31623$ and $N=10^{5}+1$ have been added. In all cases the excellent collapse of all data across three orders of magnitude in $N$ strongly confirms the validity of the measured (non-universal) exponents in the large- $N$ limit. Similar excellent collapses are also obtained for temperature profiles. Note that if a different anomaly exponent is used in the previous scaling plots (e.g. $\alpha=1 / 3$ ) no good collapse is obtained, as observed in e.g. Fig. $27 \mathrm{~b}$ above, even if we restrict the plot to the largest values of $N$. These observations thus discard the possibility of a running anomaly exponent (at least within our stringent precision limits), demonstrating the robustness of the measured anomaly exponent $\alpha$.

To end this section, we investigate the density dependence of the heat conductivity $\kappa_{L}(\rho, T)=L^{\alpha} \sqrt{T / m} k(\rho)$, see Eq. (69). Interestingly, the dynamics of the $1 d$ hard-point fluid can be shown to remain invariant under the rescaling of different observables (e.g. temperature, velocities, space, mass, etc.) [74]. Such invariance can be then used to prove rigorously that $\kappa_{L}(\rho, T)=\sqrt{T / m} f(N, \mu)$, with $f$ some adimensional function of $N$ and $\mu$. This in turn implies, via dimensional analysis, that necessarily $k(\rho)=a(\mu) \rho^{\alpha}$, with $a(\mu)$ some $\mu$-dependent amplitude. This is fully confirmed in local measurements of the density dependence of the heat conductivity, see Fig. 25b. In fact one can easily show from Eq. (69) that

$$
k[\rho(x)]=\frac{J \sqrt{m}}{\sqrt{T(x)}\left|T^{\prime}(x)\right|} L^{-\alpha},
$$

so for each set $\left(N, T_{0}, \eta\right)$ and fixed $\mu$ we performed discrete derivatives of bulk temperature profile to evaluate $T^{\prime}(x)$ and plotted the previous expression, with $J$ the heat current measured in each case, as a function of the associated $\rho(x)$. Figure $25 \mathrm{~b}$ shows the curves $k(\rho)$ so obtained for different $\mu$, which display the best collapse when the measured exponent $\alpha(\mu)$ is used. Interestingly the resulting scaling functions, though somewhat noisy due to discretization effects, exhibit a clear power-law behavior, $k(\rho)=a \rho^{\beta}$, and the fitted exponent is fully compatible in all cases with the measured anomaly exponent, $\beta=\alpha(\mu)$, see Fig. 28a above and Table 1. This shows that the density dependence of the heat conductivity in the $1 d$ diatomic hard-point gas does reflect the transport anomaly.

The above observation that $k(\rho)=a \rho^{\alpha}$ opens now the door to a full solution of the macroscopic heat transport problem for this model, written in terms of the external control parameters, namely $T_{0}, T_{L}, \eta$ and $L$, together with $\alpha$ and $a$. In fact, recalling that $G^{\prime}(\rho)=$ $k(\rho) \rho^{-5 / 2}$, we obtain that $G(\rho)=v^{*}\left(1-\rho^{\alpha-3 / 2}\right)$, with $v^{*} \equiv a /\left(\frac{3}{2}-\alpha\right)$ and where we have chosen an arbitrary constant such that $F(0)=1=G^{-1}(0)$. The universal master curve hence reads

$$
F(z)=\left(1-\frac{z}{v^{*}}\right)^{\frac{2}{2 \alpha-3}}
$$

This prediction is compared with the measured master curves in Fig. 28b, and the agreement is excellent for all mass ratios $\mu$. Eq. (75) implies in turn that density profiles can be written as

$$
\rho(x)=\left[\left(\frac{P}{T_{0}}\right)^{\alpha-\frac{3}{2}}-\frac{\psi}{v^{*}} L^{-\alpha} x\right]^{\frac{2}{2 \alpha-3}},
$$

see also Eq. (72), while temperature profiles simply follow from $T(x)=P / \rho(x)$, namely

$$
T(x)=\left[T_{0}^{\frac{3}{2}-\alpha}-\frac{J \sqrt{m}}{v^{*} P^{\alpha}} L^{-\alpha} x\right]^{\frac{2}{3-2 \alpha}} .
$$


The calculation is completed by expressing the heat current $J$ and the pressure $P$ in terms of the external parameters by using Eq. (71) above. This yields

$$
\begin{aligned}
P & =\eta\left(\frac{\frac{1}{2}-\alpha}{\frac{3}{2}-\alpha}\right)\left(\frac{T_{0}^{3 / 2-\alpha}-T_{L}^{3 / 2-\alpha}}{T_{0}^{1 / 2-\alpha}-T_{L}^{1 / 2-\alpha}}\right), \\
J & =\frac{a \eta^{\alpha}\left(\frac{1}{2}-\alpha\right)^{\alpha}}{L^{1-\alpha} \sqrt{m}\left(\frac{3}{2}-\alpha\right)^{1+\alpha}} \frac{\left(T_{0}^{3 / 2-\alpha}-T_{L}^{3 / 2-\alpha}\right)^{1+\alpha}}{\left(T_{0}^{1 / 2-\alpha}-T_{L}^{1 / 2-\alpha}\right)^{\alpha}} .
\end{aligned}
$$

The last equation for the current can be rewritten as $J=\eta^{\alpha} L^{\alpha-1} m^{-1 / 2} T_{L}^{3 / 2} h_{\alpha}\left(T_{0} / T_{L}\right)$, with

$$
h_{\alpha}(z) \equiv a \frac{\left(\frac{1}{2}-\alpha\right)^{\alpha}}{\left(\frac{3}{2}-\alpha\right)^{1+\alpha}} \frac{\left(z^{3 / 2-\alpha}-1\right)^{1+\alpha}}{\left(z^{1 / 2-\alpha}-1\right)^{\alpha}} .
$$

These detailed predictions are fully confirmed by simulations data (not shown [39]). As a self-consistent check, note that in the equilibrium limit $T_{0} \rightarrow T_{L}$ both the pressure and the heat current converge to their expected value, namely $P=\eta T_{L}$ and $J=0$.

Some comments are now in order. The excellent collapse of our data confirms that anomalous Fourier's law (68) holds in this model with a well-defined (albeit size-dependent) conductivity functional $\kappa_{L}(\rho, T)=a(\rho L)^{\alpha} \sqrt{T / m}$. This is true even for finite $N$ (as small as $\mathcal{O}\left(10^{2}\right)$ !) and under large temperature gradients, extending the range of validity of anomalous Fourier's law deep into the nonlinear regime and evidencing the absence of higher-order (Burnett-like) corrections in $1 d$ [33]. Moreover, we provide strong evidences supporting the breakdown of universality in anomalous Fourier's law for $1 d$ momentum-conserving systems [160] by showing with high accuracy that the anomaly exponent $\alpha$ for the heat conductivity of the $1 d$ diatomic hard-point fluid depends on the mass ratio $\mu$ between neighboring particles. This clear-cut observation calls into question the universality picture for heat transport based on renormalization-group and mode-coupling calculations [25,121]. This universality breakdown here reported may hint at the possible existence of hidden slowly-evolving fields in the diatomic hard-point gas other than the standard hydrodynamic ones. Remarkably, such intriguing behavior has been already observed in the nonequilibrium response of this $1 d$ model to a shock wave excitation [26,144], and suggests that an alternative fluctuating hydrodynamics description (including the additional slow fields, as in granular fluids [161]) may be needed to understand anomalous transport in this model. It has been recently suggested [126] that the existence of further slowly-evolving fields may give rise to an discrete (Fibonacci) family of anomaly exponents that can coexist in different regions of parameter space for a given model [126], changing from one value to another as a control parameter is varied, a behavior reminiscent of our results.

\section{Discussion and Outlook}

In this work we have reviewed some recent results on key open problems in the physics of transport, using both extensive numerical simulations of hard particle systems and a novel scaling method. These problems range from local equilibrium and Fourier's law to the transition to convective flow in the presence of gravity, the efficiency of boundary dissipation against bulk driving, or the universality of anomalous transport in low dimensions. As usual in science, our results lead also to new interesting questions, opening avenues of future research that we briefly discuss below. 
Our first step into the nonequilibrium realm has consisted in probing numerically the local thermodynamic equilibrium (LTE) hypothesis in a nonequilibrium hard disks fluid. We have found that macroscopic LTE is a very robust property, arbitrarily far from equilibrium and even under strong finite-size effects. This is due to a remarkable bulk-boundary structural decoupling by which all sorts of finite-size and boundary corrections are renormalized into effective boundary conditions for the bulk fluid, which in turn obeys the macroscopic laws. We have used these properties to measure with high accuracy the hard-disks equation of state in nonequilibrium simulations, obtaining excellent results even across the fluid-hexaticsolid transition regime. In particular, we have found an interesting nonequilibrium fluid/solid coexistence under a strong temperature gradient, a Stefan-like phenomenon which can be confronted with recent theoretical predictions [83-86] and calls for further investigation. Indeed, hints of the double phase transition in hard disks can be observed in the transport properties of the nonequilibrium fluid [32], a behavior that deserves further exploration. In addition, weak but clear violations of microscopic or statistical LTE have been observed in the fluctuations of the total energy, which strongly suggest that the nonequilibrium potential governing the driven fluidâs macroscopic behavior is intrinsically nonlocal. This suggests to investigate more in depth the large deviation statistics of relevant observables (currents, densities, etc.) in hard disks, using two new powerful tools that have appeared in recent years, namely macroscopic fluctuation theory (MFT) [5] and advanced simulation techniques for rare (large-deviation) events [63,162-165]. Note that the MFT approach needs a general, nonlinear formulation of fluctuating hydrodynamics for systems with several conservation laws, as recently obtained for stochastic lattice gases [166].

We have also explored the scaling properties of the inhomogeneous hydrodynamic profiles that a fluid develops in the steady state when driven out of equilibrium by a temperature gradient, showing that they obey strikingly simple scaling laws. For the hard disks fluid this implies that both density and temperature profiles in the steady state follow from two master curves, independent of the driving force and the system parameters, after a simple linear scaling of space in terms of the flowing heat current. Extensive simulations of hard disks have confirmed these scaling laws even under strong temperature gradients, implying that Fourier's law remains valid even in highly nonlinear regimes, with putative (Burnett-like) corrections absorbed into a nonlinear conductivity functional. This scaling method also offers a precise technique to determine transport coefficients from nonequilibrium simulations, and we have used it to confirm and characterize empirically the marginal anomaly of the heat conductivity predicted for hard disks. Similar, albeit more complex, scaling laws are expected to hold in sheared fluids and combined-flow situations, a property that can be used to characterize transport in driven fluids with unprecedented accuracy using nonequilibrium simulations.

By switching on a gravity field $g$ opposite to the direction of the temperature gradient, we also explored numerically, from a microscopic point of view, the transition to convective flow in hard disks. We found that the hydrodynamic kinetic energy $e_{u}(M)$ defines a proper order parameter for the convection transition: it grows steeply but continuously from zero to some nontrivial value at a critical hot bath temperature where the hydrodynamic velocity field develops structure. This critical temperature, which turns out to be consistent with the temperature of minimum relative kinetic energy fluctuations, signals the onset of convection for the different values of $g$ in the hard disks fluid. Interestingly we find that, even when convection kicks in, gravity can result in hindered heat transport as compared to the gravityfree case. It is at the second (higher) critical temperature that a percolation transition of streamlines connecting the hot and cold baths triggers efficient convective heat transport. This remarkable connection with percolation theory thus uncovers an interesting two-steps scenario for the convection instability, with two different convective transport regimes: (i) 
a semi-convective regime where coherent local motions emerge but within disconnected patches, leading to inefficient heat transport, and (ii) a fully-convective regime where a continuous percolation transition of active patches connecting the hot and cold reservoirs leads to efficient heat transport. The question remains as to whether the semi-convective regime, found here in finite-size simulations, survives in the thermodynamic $N \rightarrow \infty$ limit. In addition, and in the spirit of the scaling laws already derived for Fourier's heat transport, we have demonstrated the existence of universal master curves for hydrodynamic fields in convective flow. It would be interesting to extend this scaling picture, which has been shown to hold asymptotically in the limit of large hot bath temperatures, to intermediate temperature regimes.

We also address numerically the efficiency of boundary heat baths to dissipate the energy provided by a bulk driving mechanism. This a classical problem in nonequilibrium statistical physics with no clear answer, as the external field tends to align particle trajectories and dissipation in this particular case is a boundary effect competing with bulk driving. To answer this question, we carried out simulations of hard disks driven by an external constant field and in contact with boundary Gaussian heat baths, that provide a (deterministic) thermostatting mechanism capable of thermalizing a particle system while preserving the time reversibility of microscopic dynamics. We found that, although the response of the hard disks fluid to the external electric field is in general nonlinear, for small enough driving Ohm's law of electrical conduction is recovered, and the observed behavior is compatible with the existence of a welldefined steady state of constant average kinetic energy and particle current.

Finally, we have departed from the hard disks model to study the universality of anomalous transport in $1 d$ systems. For that, we studied numerically the diatomic hard-point gas, a paradigmatic model of anomalous heat conduction widely investigated in literature [22-24,149-156]. Using an anomalous variant of the scaling method already employed to characterize Fourier's law in hard disks, we were able to measure with unprecedented precission the anomaly exponent $\alpha$ that characterizes superdiffusive heat transport in $1 d$, see Sect. 9. We find that the measured anomaly exponent depends unambiguously on the mass ratio between neighboring particles that characterizes the diatomic hard-point gas, demonstrating the breakdown of universality in anomalous Fourier's law for 1d momentum-conserving systems [39]. This calls into question the universality picture for heat transport, and hints at the possible existence of hidden slowly-evolving fields in the diatomic hard-point gas other than the standard hydrodynamic ones. Moreover, the excellent collapses found for our hydrodynamic data confirm that anomalous Fourier's law holds in this model arbitrarily far from equilibrium, with a well-defined (albeit size-dependent) conductivity functional. The question remains as to how to reconcile the local nature of Fourier's law with the nonlocal $L^{\alpha}$-term in the conductivity $\kappa_{L}(\rho, T)$. Our data suggest that this could be achieved in a nonlinear fluctuating hydrodynamics description of the problem derived via an anomalous, non-diffusive hydrodynamic scaling of microscopic spatiotemporal variables, $x \rightarrow x / L^{1-\alpha}$ and $t \rightarrow t / L^{2-3 \alpha}$. Finally, it would be interesting to apply the scaling method here employed to other paradigmatic models of heat transport in low dimensions, as e.g. the Fermi-PastaUlam-Tsingou model of anharmonic oscillators and the hard-square or -shoulder potentials $[8,74,87,119]$, where the reported universality breakdown can be further studied. The role of conservative noise $[167,168]$ as a smoothing mechanism to get rid of non-hydrodynamic, hidden slow fields should be also investigated.

As a final remark and overall conclusion of this review paper, we want to stress how carefully-crafted numerical simulations of simple hard particle systems, together with a detailed analysis of the resulting data, open the door to unexpected discoveries in the physics of transport, paving the way to further advances in nonequilibrium physics. 
Acknowledgements We thank G. Gallavotti, J.L. Lebowitz and J. del Pozo for illuminating discussions. Financial support from Spanish Ministry MINECO Project FIS2017-84256-P is also acknowledged. We are also grateful for the computational resources and assistance provided by PROTEUS, the supercomputing center of the Institute Carlos I for Theoretical and Computational Physics at the University of Granada, Spain.

\section{Appendix A: Virial Pressure for Hard Disks}

In this Appendix we use the virial theorem to obtain the pressure field $P(x, y)$ for a system of hard disks. We thus consider a square spatial region of side $L$ where $N$ interacting disks of mass $m$ evolve. The virial theorem states

$$
\left\langle\sum_{i=1}^{N} \mathbf{r}_{i} \cdot \mathbf{F}_{i}\right\rangle=-2\left\langle E_{c}\right\rangle
$$

where $\mathbf{r}_{i}$ is the vector position of particle $i, \mathbf{F}_{i}$ is the total force acting on particle $i, E_{c}$ is the system kinetic energy and $\langle\cdot\rangle$ stands for an equilibrium ensemble average. Just as a reminder, a simple proof of the virial theorem can be given in terms of the ergodic hypothesis, namely

$$
\begin{aligned}
\left\langle\sum_{i=1}^{N} \mathbf{r}_{i} \cdot \mathbf{F}_{i}\right\rangle & =\lim _{\tau \rightarrow \infty} \frac{1}{\tau} \int_{0}^{\tau} d t \sum_{i=1}^{N} \mathbf{r}_{i}(t) \cdot \mathbf{F}_{i}(t)=\lim _{\tau \rightarrow \infty} \frac{m}{\tau} \int_{0}^{\tau} d t \sum_{i=1}^{N} \mathbf{r}_{i}(t) \cdot \frac{d^{2} \mathbf{r}_{i}(t)}{d t^{2}} \\
& =-\lim _{\tau \rightarrow \infty} \frac{m}{\tau} \int_{0}^{\tau} d t \sum_{i=1}^{N}\left(\frac{d \mathbf{r}_{i}}{d t}\right)^{2}=-2\left\langle E_{c}\right\rangle,
\end{aligned}
$$

where integration by parts has been used in the second line of the previous equation, neglecting boundary terms. The force $\mathbf{F}_{i}(t)$ acting over particle $i$ at time $t$ can be written as the sum of two terms, an internal force due to its interaction with all other particles and an external component resulting from the effect of boundaries, i.e.

$$
\mathbf{F}_{i}=\sum_{j \neq i} \mathbf{F}_{i j}+\mathbf{F}_{i}^{e x t}
$$

Assuming that the external force only acts at the system boundaries and its direction is perpendicular to the box sides with constant magnitude $F$, we have

$$
\left\langle\sum_{i=1}^{N} \mathbf{r}_{i} \cdot \mathbf{F}_{i}^{e x t}\right\rangle=\left(x_{L}-x_{0}-y_{L}+y_{0}\right) \tilde{F}=-2 L \tilde{F}=-2 S P
$$

where $x_{0, L}$ and $y_{0, L}$ are the coordinates of the box sides, $\tilde{F}$ is the average total force applied on each side, $S=L^{2}$, and we have introduced the mechanical pressure $P \equiv \tilde{F} / L$. Note that the average force $\tilde{F}$ will be constant for systems in mechanical equilibrium. On the other hand, the interparticle force contribution can be written as

$$
\left\langle\sum_{i=1}^{N} \mathbf{r}_{i} \cdot \sum_{j \neq i} \mathbf{F}_{i j}\right\rangle=\frac{1}{2} \sum_{i, j}\left\langle\left(\mathbf{r}_{i}-\mathbf{r}_{j}\right) \cdot \mathbf{F}_{i j}\right\rangle=\sum_{\langle i, j\rangle}\left\langle\mathbf{r}_{i j} \cdot \mathbf{F}_{i j}\right\rangle
$$

where we have used Newton's third law, $\mathbf{F}_{i j}=-\mathbf{F}_{j i}$, we defined $\mathbf{r}_{i j}=\mathbf{r}_{i}-\mathbf{r}_{j}$, and $\langle i, j\rangle$ is the set of all different pairs of particles. For hard disks interactions only happen when a pair of particles collide at contact. Assuming that the collision occurs in a infinitesimal time 
interval $2 \epsilon$, we can write $\mathbf{F}_{i j}$ as the variation of the linear momentum on such time interval, i.e.

$$
\mathbf{F}_{i j}\left(t_{n}\right) \simeq \frac{1}{2 \epsilon}\left[\mathbf{p}_{i}\left(t_{n}+\epsilon\right)-\mathbf{p}_{i}\left(t_{n}-\epsilon\right)\right]
$$

where $t_{n}$ is the collision time. Due to the elastic character of the collision, the disks' linear momentum components along the vector connecting their centers are exchanged, so $\mathbf{r}_{i j}$. $\mathbf{p}_{i}\left(t_{n}-\epsilon\right)=\mathbf{r}_{i j} \cdot \mathbf{p}_{j}\left(t_{n}+\epsilon\right)$ and therefore

$$
\mathbf{r}_{i j} \cdot \mathbf{F}_{i j}=\frac{1}{2 \epsilon}\left(\mathbf{r}_{i j} \cdot \mathbf{p}_{i}\left(t_{n}+\epsilon\right)-\mathbf{r}_{i j} \cdot \mathbf{p}_{i}\left(t_{n}-\epsilon\right)\right)=\frac{1}{2 \epsilon} \mathbf{r}_{i j} \cdot \mathbf{p}_{i j}
$$

where $\mathbf{p}_{i j}=\mathbf{p}_{i}\left(t_{n}+\epsilon\right)-\mathbf{p}_{j}\left(t_{n}+\epsilon\right)$. In this way, we can finally write, see Eq. (A5),

$$
\left\langle\sum_{i=1}^{N} \mathbf{r}_{i} \cdot \sum_{j \neq i} \mathbf{F}_{i j}\right\rangle=\lim _{\tau \rightarrow \infty} \frac{1}{\tau} \sum_{n: t_{n} \in[0, \tau]} \mathbf{r}_{i j} \cdot \mathbf{p}_{i j}
$$

where the sum is over all the pair collisions occurring in the time interval $[0, \tau]$. The virial theorem for hard disks can be hence written as

$$
-2 S P+\lim _{\tau \rightarrow \infty} \frac{1}{\tau} \sum_{n: t_{n} \in[0, \tau]} \mathbf{r}_{i j} \cdot \mathbf{p}_{i j}=-2 N T
$$

where we have used that in equilibrium $\left\langle E_{c}\right\rangle=N T$, with $T$ the system temperature. Finally the virial pressure for hard disks can be expressed as

$$
P=\frac{\rho T}{\pi \ell^{2}}+\lim _{\tau \rightarrow \infty} \frac{1}{2 S \tau} \sum_{n: t_{n} \in[0, \tau]} \mathbf{r}_{i j} \cdot \mathbf{p}_{i j}
$$

where $\rho=N \pi \ell^{2} / S$ and we recall that $\ell$ is the disk radius.

\section{References}

1. Balescu, R.: Equilibrium and Nonequilibrium Statistical Mechanics. Wiley, New York (1975)

2. Pathria, R., Beale, P.: Statistical Mechanics. Academic Press, New York (2009)

3. Livi, R., Politi, P.: Nonequilibrium Statistical Physics: A Modern Perspective. Cambridge University Press, Cambridge (2017)

4. Derrida, B.: Non-equilibrium steady states: fluctuations and large deviations of the density and of the current. J. Stat. Mech. 2007(7), P07023 (2007)

5. Bertini, L., Sole, A.D., Gabrielli, D., Jona-Lasinio, G., Landim, C.: Macroscopic fluctuation theory. Rev. Mod. Phys. 87, 593 (2015)

6. De Groot, S., Mazur, P.: Non-Equilibrium Thermodynamics, Dover Books on Physics. Dover Publications, New York (2013)

7. Fourier, J.: Théorie analytique de la chaleur (Chez Firmin Didot, père et fils, 1822)

8. Bonetto, F., Lebowitz, J.L., Rey-Bellet, L.: Mathematical Physics 2000, (Imperial College Press, London, : Chap, p. 128. A Challenge for Theorists, Fourier's Law (2000)

9. Mulero, A.: Theory and Simulation of Hard-Sphere Fluids and Related Systems, Lecture Notes in Physics, vol. 753. Springer (2008)

10. Chaikin, P.M., Lubensky, T.C.: Principles of Condensed Matter Physics, vol. 1. Cambridge University Press, Cambridge (2000)

11. Cates, M.E., Evans, M.R. (eds.): Soft and Fragile Matter: Nonequilibrium Dynamics, Metastability and Flow. Institute of Physics, Bristol (2000) 
12. Berthier, L., Biroli, G., Bouchaud, J.P., Cipelletti, L., van Saarloos, W. (eds.): Dynamical Heterogeneities in Glasses, Colloids and Granular Materials. Oxford University Press, Oxford (2011)

13. de Gennes, P .G., Prost, J.: The Physics of Liquid Crystals. Oxford University Press, Oxford (1993)

14. Mehta, A.: Granular Physics. Cambridge University Press, Cambridge (2007)

15. Alder, B.J., Wainwright, T.E.: Studies in molecular dynamics 1 . General method. J. Chem. Phys. 31, 459 (1959)

16. Alder, B.J., Wainwright, T.E.: Phase transition in elastic disks. Phys. Rev. 127, 359 (1962)

17. Alder, B.J., Wainwright, W.: Velocity autocorrelations for hard spheres. Phys. Rev. Lett. 18, 988 (1967)

18. Alder, B.J., Wainwright, W.: Decay of velocity autocorrelation function. Phys. Rev. A 1, 18 (1970)

19. Résibois, P.M., De Leener, M.: Classical Kinetic Theory of Fluids. Wiley, New York (1977)

20. Rosenbluth, M.N., Rosenbluth, A.W.: Further results on Monte-Carlo equations of state. J. Chem. Phys. 22, 881 (1954)

21. Dhar, A.: Heat conduction in a one-dimensional gas of elastically colliding particles of unequal masses. Phys. Rev. Lett. 86, 3554 (2001)

22. Garrido, P.L., Hurtado, P.I., Nadrowski, B.: Simple one-dimensional model of heat conduction which obeys Fourier's law. Phys. Rev. Lett. 86, 5486 (2001)

23. Savin, A.V., Tsironis, G.P., Zolotaryuk, A.V.: Heat conduction in one-dimensional systems with hardpoint interparticle interactions. Phys. Rev. Lett. 88, 154301 (2002)

24. Grassberger, P., Nadler, W., Yang, L.: Heat conduction and entropy production in a one-dimensional hard-particle gas. Phys. Rev. Lett. 89, 180601 (2002)

25. Narayan, O., Ramaswamy, S.: Anomalous heat conduction in one-dimensional momentum-conserving systems. Phys. Rev. Lett. 89, 200601 (2002)

26. Hurtado, P.I.: Breakdown of hydrodynamics in a simple one-dimensional fluid. Phys. Rev. Lett. 96, 010601 (2006)

27. Hurtado, P.I., Pérez-Espigares, C., del Pozo, J.J., Garrido, P.L.: Symmetries in fluctuations far from equilibrium. Proc. Natl. Acad. Sci. USA 108, 7704 (2011)

28. Chernov, N.I., Lebowitz, J.L.: Stationary nonequilibrium states in boundary-driven Hamiltonian systems: shear flow. J. Stat. Phys. 86, 953 (1997)

29. Bonetto, F., Gallavotti, G., Garrido, P.L.: Chaotic principle: an experimental test. Physica D 105, 226 (1997)

30. Garrido, P.L., Goldstein, S., Lebowitz, J.L.: Boltzmann entropy for dense fluids not in local equilibrium. Phys. Rev. Lett. 92, 050602 (2004)

31. Szász, D. (ed.): Hard Ball Systems and the Lorentz Gas. Springer, Berlin (2000)

32. del Pozo, J.J., Garrido, P.L., Hurtado, P.I.: Probing local equilibrium in nonequilibrium fluids. Phys. Rev. E 92, 022117 (2015a)

33. del Pozo, J.J., Garrido, P.L., Hurtado, P.I.: Scaling laws and bulk-boundary decoupling in heat flow. Phys. Rev. E 91, 032116 (2015b)

34. Gass, D.M.: Enskog theory for a rigid disk fluid. J. Chem. Phys. 54, 1898 (1971)

35. Andersen, H.C., Weeks, J.D., Chandler, D.: Relationship between hard-sphere fluid and fluids with realistic repulsive forces. Phys. Rev. A 4, 1597 (1971)

36. Barker, J.A., Henderson, D.: What is liquid-Understanding states of matter. Rev. Mod. Phys. 48, 587 (1976)

37. Marín, M., Risso, D., Cordero, P.: Efficient algorithms for many-body hard particle molecular-dynamics. J. Comp. Phys. 109, 306 (1993)

38. Isobe, M.: Hard sphere simulation in statistical physics-methodologies and applications. Mol. Simul. 42, 1317 (2016)

39. Hurtado, P.I., Garrido, P.L.: A violation of universality in anomalous Fourier's law. Sci. Rep. 6, 38823 (2016)

40. Garrido, P.L.: Rayleigh-Bénard convection in a hard disk system (2018). arXiv:1802.09759

41. Garrido, P.L., Gallavotti, G.: Boundary dissipation in a driven hard disk system. J. Stat. Phys. 126, 1201 (2007)

42. Cordero, P., Marín, M., Risso, D.: Efficient simulations of microscopic fluids: Algorithm and experiments. Chaos Solitons Fractals 6, 95 (1995)

43. Rapaport, D.C.: The event-driven approach to N-body simulation. Prog. Theoret. Phys. Suppl. 178, 5 (2009)

44. Bernard, E.P., Krauth, W.: Two-step melting in two dimensions: first-order liquid-hexatic transition. Phys. Rev. Lett. 107, 155704 (2011)

45. Isobe, M.: Long-time tail of the velocity autocorrelation function in a two-dimensional moderately dense hard-disk fluid. Phys. Rev. E 77, 021201 (2008) 
46. Santos, A., de López Haro, M., Bravo Yuste, S.: An accurate and simple equation of state for hard disks. J. Chem. Phys 103, 4622 (1995)

47. Henderson, D.: A simple equation of state for hard discs. Mol. Phys. 30, 971 (1975)

48. Martin-Lof, A.: Statistical Mechanics and the Foundations of Thermodynamics, Lecture Notes in Physics, vol. 101. Springer, Berlin (1979)

49. Gallavotti, G.: Foundations of Fluid Dynamics. Springer, Berlin (2002)

50. Gallavotti, G.: Nonequilibrium and Irreversibility, Theoretical and Mathematical Physics. Springer, New York (2014)

51. Evans, D.J., Morriss, G.: Statistical Mechanics of Nonequilibrium Liquids. Cambridge University Press, Cambridge (2008)

52. Lebowitz, J.L.: Macroscopic laws, microscopic dynamics, times arrow and boltzmann entropy. Physica A 194, 1 (1993)

53. Chernov, N.I., Eyink, G.L., Lebowitz, J.L., Sinai, Y.G.: Derivation of Ohm law in a deterministic mechanical model. Phys. Rev. Lett. 70, 2209 (1993a)

54. Chernov, N.I., Eyink, G.L., Lebowitz, J.L., Sinai, Y.G.: Steady-state electrical-conduction in the periodic Lorentz gas. Commun. Math. Phys. 154, 569 (1993b)

55. Gallavotti, G., Cohen, E.G.D.: Dynamical ensembles in nonequilibrium statistical mechanics. Phys. Rev. Lett. 74, 2694 (1995a)

56. Gallavotti, G., Cohen, E.G.D.: Dynamical ensembles in stationary states. J. Stat. Phys. 80, 931 (1995b)

57. Gallavotti, G.: Ergodicity, ensembles, irreversibility in Boltzmann and beyond. J. Stat. Phys. 78, 1571 (1995)

58. Gallavotti, G.: Chaotic hypothesis: onsager reciprocity and fluctuation-dissipation theorem. J. Stat. Phys. 84, 899 (1996)

59. Chernov, N.I., Lebowitz, J.L.: Stationary shear flow in boundary driven hamiltonian systems. Phys. Rev. Lett. 75, 2831 (1995)

60. Spohn, H.: Large Scale Dynamics of Interacting Particles. Theoretical and Mathematical Physics. Springer, Berlin (2012)

61. Landau, L., Lifshitz, E.: Fluid Mechanics, vol. 6. Elsevier, Oxford (2013)

62. de Zarate, J.O., Sengers, J.: Hydrodynamic Fluctuations in Fluids and Fluid Mixtures, Hydrodynamic Fluctuations in Fluids and Fluid Mixtures. Elsevier, Amsterdam (2006)

63. Hurtado, P.I., Espigares, C.P., del Pozo, J.J., Garrido, P.L.: Thermodynamics of currents in nonequilibrium diffusive systems: theory and simulation. J. Stat. Phys. 154, 214 (2014)

64. Hurtado, P.I., Garrido, P.L.: Test of the additivity principle for current fluctuations in a model of heat conduction. Phys. Rev. Lett. 102, 250601 (2009)

65. Hurtado, P.I., Garrido, P.L.: Spontaneous symmetry breaking at the fluctuating level. Phys. Rev. Lett. 107, 180601 (2011)

66. Prados, A., Lasanta, A., Hurtado, P.I.: Large fluctuations in driven dissipative media. Phys. Rev. Lett. 107, 140601 (2011)

67. Touchette, H.: The large deviation approach to statistical mechanics. Phys. Rep. 478, 1 (2009)

68. Derrida, B., Lebowitz, J.L., Speer, E.R.: Free energy functional for nonequilibrium systems: an exactly solvable case. Phys. Rev. Lett. 87, 150601 (2001)

69. Derrida, B., Lebowitz, J.L., Speer, E.R.: Large deviation of the density profile in the steady state of the open symmetric simple exclusion process. J. Stat. Phys. 107, 599 (2002)

70. Derrida, B., Lebowitz, J.L., Speer, E.R.: Exact large deviation functional of a stationary open driven diffusive system: the asymmetric exclusion process. J. Stat. Phys. 110, 775 (2003)

71. Bertini, L., Gabrielli, D., Lebowitz, J.L.: Large deviations for a stochastic model of heat flow. J. Stat. Phys. 121, 843 (2005)

72. Garrido, P.L., Lebowitz, J.L., Maes, C., Spohn, H.: Long-range correlations for conservative dynamics. Phys. Rev. A 42, 1954 (1990)

73. Eckmann, J.P., Young, L.S.: Temperature profiles in Hamiltonian heat conduction. Europhys. Lett. 68, 790 (2004)

74. Dhar, A.: Heat transport in low-dimensional systems. Adv. Phys. 57, 457 (2008)

75. Tenenbaum, A., Ciccotti, G., Gallico, R.: Stationary non-equilibrium states by molecular-dynamicsfourier law. Phys. Rev. A 25, 2778 (1982)

76. Henderson, D.: Monte-carlo and perturbation-theory studies of equation of state of 2-dimensional lennard-jones fluid. Mol. Phys. 34, 301 (1977)

77. Engel, M., Anderson, J.A., Glotzer, S.C., Isobe, M., Bernard, E.P., Krauth, W.: Hard-disk equation of state: first-order liquid-hexatic transition in two dimensions with three simulation methods. Phys. Rev. E 87, 042134 (2013) 
78. Metropolis, N., Rosenbluth, A.W., Rosenbluth, M.N., Teller, A.H., Teller, E.: Equation of state calculations by fast computing machines. J. Chem. Phys. 21, 1087 (1953)

79. Alder, B.J., Hoover, W.G., Young, D.A.: Studies in molecular dynamics. V. High-density equation of state and entropy for hard disks and spheres. J. Chem. Phys. 49, 3688 (1968)

80. Erpenbeck, J.J., Luban, M.: Equation of state of the classical hard-disk fluid. Phys. Rev. A 32, 2920 (1985)

81. Mak, C.H.: Large-scale simulations of the two-dimensional melting of hard disks. Phys. Rev. E 73, 065104 (2006)

82. Kolafa, J., Rottner, M.: Simulation-based equation of state of the hard disk fluid and prediction of higher-order virial coefficients. Mol. Phys. 104, 3435 (2006)

83. De Masi, A., Presutti, E., Tsagkarogiannis, D.: Fourier law, phase transitions and the stationary stefan problem. Arch. Ration. Mech. Anal. 201, 681 (2011)

84. Nakagawa, N., Sasa, S.-I.: Liquid-gas transitions in steady heat conduction. Phys. Rev. Lett. 119, 260602 (2017)

85. Sasa, S.-I., Nakagawa, N., Itami, M., Nakayama, Y.: Stochastic order parameter dynamics for phase coexistence in heat conduction (2019). arXiv:1908.03029

86. Nakagawa, N., Sasa, S.-I.: Global thermodynamics for heat conduction systems (2019). arXiv:1906.07022

87. Lepri, S., Livi, R., Politi, A.: Thermal conduction in classical low-dimensional lattices. Phys. Rep. 377, 1 (2003)

88. Garrido, P.L., Hurtado, P.I.: Comment on Simple one-dimensional model of heat conduction which obeys Fourier's law- Reply. Phys. Rev. Lett. 89, 079402 (2002a)

89. Garrido, P.L., Hurtado, P.I.: Comment on Simple one-dimensional model of heat conduction which obeys Fourier's law-reply. Phys. Rev. Lett. 88, 249402 (2002b)

90. Garrido, P.L., Hurtado, P.I., Tizón-Escamilla, N.: Infinite family of universal profiles for heat current statistics in Fourier's law. Phys. Rev. E 99, 022134 (2019)

91. Gnan, N., Schroder, T.B., Pedersen, U.R., Bailey, N.P., Dyre, J.C.: Pressure-energy correlations in liquids. IV. Isomorphs in liquid phase diagrams. J. Chem. Phys. 131, 234504 (2009)

92. Santos, A.: Chemical-potential route: a hidden Percus-Yevick equation of state for hard spheres. Phys. Rev. Lett. 109, 120601 (2012)

93. García-Rojo, R., Luding, S., Brey, J.J.: Transport coefficients for dense hard-disk systems. Phys. Rev. E 74, 061305 (2006)

94. An animated explanation of the scaling procedure can be found here credit: J. del Pozo, (2014). https:// ic1.ugr.es/members/phurtado/wp-content/uploads/sites/3/2019/10/scalin.mp4

95. Dorfman, J.R., Kirkpatrick, T.R., Sengers, J.V.: Generic long-range correlations in molecular fluids. Ann. Rev. Phys. Chem. 45, 213 (1994)

96. Zheng, Y.H., García, A.L., Alder, B.J.: Comparison of kinetic theory and hydrodynamics for Poiseuille flow. J. Stat. Phys. 109, 495 (2002)

97. Xu, K.: Super-Burnett solutions for Poiseuille flow. Phys. Fluids 15, 2077 (2003)

98. Risso, D., Cordero, P.: Two-dimensional gas of disks: thermal conductivity. J. Stat. Phys. 82, 1453 (1996)

99. Bodenschatz, E., Pesch, W., Ahlers, G.: Recent developments in Rayleigh-Bénard convection. Annu. Rev. Fluid Mech. 32, 709 (2000)

100. Lappa, M.: Thermal Convection: Patterns, Evolution and Stability. Wiley, New York (2009)

101. Mutabazi, I., Wesfreid, J.E., Guyon, E.: Dynamics of Spatio-Temporal Cellular Structures: Henri Bénard Centenary Review, vol. 207. Springer, New York (2010)

102. Mareschal, M., Kestemont, E.: Experimental-evidence for convective rolls in finite two-dimensional molecular-models. Nature 329, 427 (1987a)

103. Mareschal, M., Kestemont, E.: Order and fluctuations in nonequilibrium molecular dynamics simulations of two-dimensional fluids. J. Stat. Phys. 48, 1187 (1987b)

104. Mareschal, M., Malek Mansour, M., Puhl, A., Kestemont, E.: Molecular dynamics versus hydrodynamics in a two-dimensional Rayleigh-Bénard system. Phys. Rev. Lett. 61, 2550 (1988)

105. Rapaport, D.C.: Molecular-dynamics study of Rayleigh-Bénard convection. Phys. Rev. Lett. 60, 2480 (1988)

106. Puhl, A., Mansour, M.M., Mareschal, M.: Quantitative comparison of molecular dynamics with hydrodynamics in Rayleigh-Bénard convection. Phys. Rev. A 40, 1999 (1989)

107. Rapaport, D.C.: Unpredictable convection in a small box: molecular-dynamics experiments. Phys. Rev. A 46, 1971 (1992)

108. Risso, D., Cordero, P.: Empirical determination of the onset of convection for a hard disk system. In: Instabilities and Nonequilibrium Structures IV, p. 199. Springer (1993)

109. Chandrasekhar, S.: Hydrodynamic and Hydromagnetic Stability. Dover Publications, New York (2013) 
110. Press, W.H., Teukolsky, S.A., Vetterling, W.T., Flannery, B.P.: Numerical Recipes in Fortran 90, vol. 2. Cambridge University Press, Cambridge (1996)

111. Stalling, D., Hege, H.C.: Fast and resolution independent line integral convolution. In: Mair, S.G., Cook, R. (eds.) Proceedings of the 22nd Annual Conference on Computer Graphics and Interactive Techniques (SIGGRAPH'95), p. 249. ACM, New York (1995)

112. Cabral, B., Leedom, L.C.: Imaging vector fields using line integral convolution. Comput. Graphic 27, 263 (1993)

113. Mertens, S., Moore, C.: Continuum percolation thresholds in two dimensions. Phys. Rev. E 86, 061109 (2012)

114. Yuge, T., Ito, N., Shimizu, A.: Nonequilibrium molecular dynamics simulation of electric conduction. J. Phys. Soc. Jpn. 74, 1895 (2005)

115. Ayton, G., Evans, D.J., Searles, D.J.: A local fluctuation theorem. J. Chem. Phys. 115, 2033 (2001)

116. Todd, B.D., Evans, D.J., Daivis, P.J.: Pressure tensor for inhomogeneous fluids. Phys. Rev. E 52, 1627 (1995)

117. Todd, B.D., Evans, D.J.: Temperature profile for Poiseuille flow, temperature profile for Poiseuille flow. Phys. Rev. E 55, 2800 (1997)

118. Lebowitz, J.L.: Nonequilibrium statistical physics today. Where shall we go from here? AIP Conf. Proc. 1332, 3 (2011)

119. Lepri, S. (ed.): Thermal Transport in Low Dimensions: From Statistical Physics to Nanoscale Heat Transfer, Lectures Notes in Physics, vol. 921. Springer (2016)

120. Liu, S., Xu, X.F., Xie, R.G., Zhang, G., Li, B.W.: Anomalous heat conduction and anomalous diffusion in low dimensional nanoscale systems. Eur. Phys. J. B 85, 1 (2012)

121. van Beijeren, H.: Exact results for anomalous transport in one-dimensional Hamiltonian systems. Phys. Rev. Lett. 108, 180601 (2012)

122. Mendl, C., Spohn, H.: Dynamic correlators of Fermi-Pasta-Ulam chains and nonlinear fluctuating hydrodynamics. Phys. Rev. Lett. 111, 230601 (2013)

123. Spohn, H.: Nonlinear fluctuating hydrodynamics for anharmonic chains. J. Stat. Phys. 154, 1191 (2014)

124. Das, S.G., Dhar, A., Saito, K., Mendl, C.B., Spohn, H.: Numerical test of hydrodynamic fluctuation theory in the Fermi-Pasta-Ulam chain. Phys. Rev. E 90, 012124 (2014)

125. Mendl, C., Spohn, H.: Current fluctuations for anharmonic chains in thermal equilibrium. J. Stat. Mech. 2015(3), P03007 (2015)

126. Popkov, V., Schadschneider, A., Schmidt, J., Schütz, G.M.: Fibonacci family of dynamical universality classes. Proc. Natl. Acad. Sci. USA 112, 12645 (2015)

127. Ghosh, S., Calizo, I., Teweldebrhan, D., Pokatilov, E., Nika, D., Balandin, A., Bao, W., Miao, F., Lau, C.N.: Extremely high thermal conductivity of graphene: prospects for thermal management applications in nanoelectronic circuits. Appl. Phys. Lett. 92, 151911 (2008)

128. Balandin, A.A., Ghosh, S., Bao, W., Calizo, I., Teweldebrhan, D., Miao, F., Lau, C.N.: Superior thermal conductivity of single-layer graphene. Nano Lett. 8, 902 (2008)

129. Ghosh, S., Bao, W., Nika, D.L., Subrina, S., Pokatilov, E.P., Lau, C.N., Balandin, A.A.: Dimensional crossover of thermal transport in few-layer graphene. Nat. Mater. 9, 555 (2010)

130. Balandin, A.A.: Thermal properties of graphene and nanostructured carbon materials. Nat. Mater. 10, 569 (2011)

131. Xu, X., Pereira, L.F.C., Wang, Y., Wu, J., Zhang, K., Zhao, X., Bae, S., Bui, C.T., Xie, R., Thong, J.T.L., et al.: Length-dependent thermal conductivity in suspended single-layer graphene. Nat. Commun. $\mathbf{5}$, 3689 (2014)

132. Meier, T., Menges, F., Nirmalraj, P., Hölscher, H., Riel, H., Gotsmann, B.: Length-dependent thermal transport along molecular chains. Phys. Rev. Lett. 113, 060801 (2014)

133. Chang, C.-W., Okawa, D., Garcia, H., Majumdar, A., Zettl, A.: Breakdown of Fourier's law in nanotube thermal conductors. Phys. Rev. Lett. 101, 075903 (2008)

134. Henry, A., Chen, C.: High thermal conductivity of single polyethylene chains using molecular dynamics simulations. Phys. Rev. Lett. 101, 235502 (2008)

135. Liu, J., Yang, R.: Length-dependent thermal conductivity of single extended polymer chains. Phys. Rev. B 86, 104307 (2012)

136. Hsiao, T.-K., Chang, H.-K., Liou, S.-C., Chu, M.-W., Lee, S.-C., Chang, C.-W.: Observation of roomtemperature ballistic thermal conduction persisting over $8.3 \mu \mathrm{m}$ in SiGe nanowires. Nat. Nanotechnol. 8, 534 (2013)

137. Yang, N., Zhang, G., Li, B.: Violation of Fourier's law and anomalous heat diffusion in silicon nanowires. Nano Today 5, 85 (2010)

138. Huang, X., Liu, G., Wang, X.: New secrets of spider silk: exceptionally high thermal conductivity and its abnormal change under stretching. Adv. Mater. 24, 1482 (2012) 
139. Lee-Dadswell, G.R.: Universality classes for thermal transport in one-dimensional oscillator systems. Phys. Rev. E 91, 032102 (2015a)

140. Delfini, L., Lepri, S., Livi, R., Politi, A.: Self-consistent mode-coupling approach to one-dimensional heat transport. Phys. Rev. E 73, 060201 (2006)

141. Delfini, L., Lepri, S., Livi, R., Politi, A.: Anomalous kinetics and transport from 1D self-consistent mode-coupling theory. J. Stat. Mech. 2007(2), P02007 (2007)

142. Delfini, L., Denisov, S., Lepri, S., Livi, R., Mohanty, P.K., Politi, A.: Energy diffusion in hard-point systems. Eur. Phys. J. Spec. Top. 146, 21 (2007b)

143. Politi, A.: Heat conduction of the hard point chain at zero pressure. J. Stat. Mech. 2011(3), P03028 (2011)

144. Hurtado, P.I.: Strong shock waves and nonequilibrium response in a one-dimensional gas: a Boltzmann equation approach. Phys. Rev. E 72, 041101 (2005)

145. Lee-Dadswell, G., Nickel, B., Gray, C.: Thermal conductivity and bulk viscosity in quartic oscillator chains. Phys. Rev. E 72, 031202 (2005)

146. Lee-Dadswell, G.: Predicting and identifying finite-size effects in current spectra of one-dimensional oscillator chains. Phys. Rev. E 91, 012138 (2015b)

147. Liu, S., Hänggi, P., Li, N., Ren, J., Li, B.: Anomalous heat diffusion. Phys. Rev. Lett. 112, 040601 (2014)

148. Li, Y., Liu, S., Li, N., Hänggi, P., Li, B.: 1D momentum-conserving systems: the conundrum of anomalous versus normal heat transport. New J. Phys. 17, 043064 (2015)

149. Casati, G.: Energy transport and the Fourier heat law in classical systems. Found. Phys. 16, 51 (1986)

150. Garrido, P.L., Marro, J.: Effective Hamiltonian description of nonequilibrium spin systems. Phys. Rev. Lett. 62, 1929 (1989)

151. Casati, G., Wang, L., Prosen, T.: A one-dimensional hard-point gas and thermoelectric efficiency. J. Stat. Mech. 2009(3), L03004 (2009)

152. Brunet, E., Derrida, B., Gerschenfeld, A.: Fluctuations of the heat flux of a one-dimensional hard particle gas. Europhys. Lett. 90, 20004 (2010)

153. Boozer, A.: Boltzmann equations for a binary one-dimensional ideal gas. Phys. Rev. E 84, 031127 (2011)

154. Mendl, C.B., Spohn, H.: Equilibrium time-correlation functions for one-dimensional hard-point systems. Phys. Rev. E 90, 012147 (2014)

155. Chen, S., Wang, J., Casati, G., Benenti, G.: Nonintegrability and the Fourier heat conduction law. Phys. Rev. E 90, 032134 (2014a)

156. Chen, S., Zhang, Y., Wang, J., Zhao, H.: Finite-size effects on current correlation functions. Phys. Rev. E 89, 022111 (2014b)

157. Bhattacharjee, S.M., Seno, F.: A measure of data collapse for scaling. J. Phys. A 34, 6375 (2001)

158. Hurtado, P.I., Redner, S.: Simplest piston problem. I. Elastic collisions. Phys. Rev. E 73, 016136 (2006a)

159. Hurtado, P.I., Redner, S.: Simplest piston problem. II. Inelastic collisions. Phys. Rev. E 73, 016137 (2006b)

160. Xiong, D., Wang, J., Zhang, Y., Zhao, H.: Nonuniversal heat conduction of one-dimensional lattices. Phys. Rev. E 85, 020102 (2012)

161. Dufty, J.W., Brey, J.J.: Choosing hydrodynamic fields. Math. Model. Nat. Phenom. 6, 19 (2011)

162. Giardinà, C., Kurchan, J., Peliti, L.: Direct evaluation of large-deviation functions. Phys. Rev. Lett. 96, 120603 (2006)

163. Lecomte, V., Tailleur, J.: A numerical approach to large deviations in continuous time. J. Stat. Mech. 2007(3), P03004 (2007)

164. Giardinà, C., Kurchan, J., Lecomte, V., Tailleur, J.: Simulating rare events in dynamical processes. J. Stat. Phys. 145, 787 (2011)

165. Pérez-Espigares, C., Hurtado, P.I.: Sampling rare events across dynamical phase transitions. Chaos 29, 083106 (2019)

166. Gutiérrez-Ariza, C., Hurtado, P.I.: The kinetic exclusion process: a tale of two fields. J. Stat. Mech. 103203 (2019)

167. Bernardin, C., Gonçalves, P., Jara, M.: 3/4-Fractional superdiffusion in a system of harmonic oscillators perturbed by a conservative noise. Arch. Ration. Mech. Anal. 220, 505 (2016)

168. Jara, M., Komorowski, T., Olla, S.: Superdiffusion of energy in a chain of harmonic oscillators with noise. Commun. Math. Phys. 339, 407 (2015)

Publisher's Note Springer Nature remains neutral with regard to jurisdictional claims in published maps and institutional affiliations. 


\section{Affiliations}

\section{Pablo I. Hurtado ${ }^{1}$ (D) Pedro L. Garrido ${ }^{1}$}

$凶$ Pablo I. Hurtado phurtado@onsager.ugr.es

Pedro L. Garrido

garrido@ onsager.ugr.es

1 Departamento de Electromagnetismo y Física de la Materia, Institute Carlos I for Theoretical and Computational Physics, Universidad de Granada, 18071 Granada, Spain 